\title{
Resolving the chemical substructure of Orion-KL $\mathrm{L}^{\star} \star \star$
}

\author{
S. Feng (冯思轶) ${ }^{1}$, H. Beuther ${ }^{1}$, Th. Henning ${ }^{1}$, D. Semenov ${ }^{1}$, A. Palau ${ }^{2}$, and E. A. C. Mills ${ }^{3}$ \\ 1 Max-Planck-Institut für Astronomie, Königstuhl 17, 69117 Heidelberg, Germany \\ e-mail: syfeng@mpia.de \\ 2 Centro de Radioastronomia y Astrofisica, Universidad Nacional Autonoma de Mexico, Morelia Michoacan, Mexico \\ 3 National Radio Astronomy Observatory, 1003 Lopezville Road, Socorro, NM 87801, USA
}

Received 22 September 2013 / Accepted 12 March 2015

\begin{abstract}
Context. The Kleinmann-Low nebula in Orion (Orion-KL) is the nearest example of a high-mass star-forming environment. Studying the resolved chemical substructures of this complex region provides important insight into the chemistry of high-mass star-forming regions (HMSFRs), as it relates to their evolutionary states.

Aims. The goal of this work is to resolve the molecular line emission from individual substructures of Orion-KL at high spectral and spatial resolution and to infer the chemical properties of the associated gas.

Methods. We present a line survey of Orion-KL obtained from combined Submillimeter Array (SMA) interferometric and IRAM $30 \mathrm{~m}$ single-dish observations. Covering a $4 \mathrm{GHz}$ bandwidth in total, this survey contains over 160 emission lines from 20 species (25 isotopologues), including 11 complex organic molecules (COMs). Spectra are extracted from individual substructures and the intensity-integrated distribution map for each species is provided. We then estimate the rotation temperature for each substructure, along with their molecular column densities and abundances.

Results. For the first time, we complement $1.3 \mathrm{~mm}$ interferometric data with single-dish observations of the Orion-KL region and study small-scale chemical variations in this region. (1) We resolve continuum substructures on $\sim 3^{\prime \prime}$ angular scale. (2) We identify lines from the low-abundance $\mathrm{COMs} \mathrm{CH}_{3} \mathrm{COCH}_{3}$ and $\mathrm{CH}_{3} \mathrm{CH}_{2} \mathrm{OH}$, as well as tentatively detect $\mathrm{CH}_{3} \mathrm{CHO}$ and long carbon-chain molecules $\mathrm{C}_{6} \mathrm{H}$ and $\mathrm{HC}_{7} \mathrm{~N}$. (3) We find that while most COMs are segregated by type, peaking either towards the hotcore (e.g., nitrogenbearing species) or the compact ridge (e.g., oxygen-bearing species like $\mathrm{HCOOCH}_{3}$ and $\mathrm{CH}_{3} \mathrm{OCH}_{3}$ ), the distributions of others do not follow this segregated structure (e.g., $\mathrm{CH}_{3} \mathrm{CH}_{2} \mathrm{OH}, \mathrm{CH}_{3} \mathrm{OH}, \mathrm{CH}_{3} \mathrm{COCH}_{3}$ ). (4) We find a second velocity component of $\mathrm{HNCO}$, $\mathrm{SO}_{2},{ }^{34} \mathrm{SO}_{2}$, and $\mathrm{SO}$ lines, which may be associated with a strong shock event in the low-velocity outflow. (5) Temperatures and molecular abundances show large gradients between central condensations and the outflow regions, illustrating a transition between hot molecular core and shock-chemistry dominated regimes.

Conclusions. Our observations of spatially resolved abundance variations in Orion-KL provide the nearest reference source for hot molecular core and outflow chemistry, which will be an important example for interpreting the chemistry of more distant HMSFRs.
\end{abstract}

Key words. stars: formation - ISM: abundances - stars: massive - ISM: lines and bands - ISM: molecules - submillimeter: ISM

\section{Introduction}

The birth sites of high-mass star-forming regions (HMSFRs) are highly complex structures, consisting of different gas and dust cores, embedded in a less dense gas envelope. While the complexity of dynamic processes within these regions has been characterized well (e.g., Zinnecker \& Yorke 2007; Beuther et al. 2007b; Tan et al. 2014), our knowledge of the chemical properties of these regions is still poor. Since specific molecules can act as tracers of physical processes (e.g., Schilke et al. 1997; Charnley et al. 1997; Bachiller et al. 2001; Viti et al. 2004; Garrod 2013), characterizing the chemistry of gas associated with the early stages of high-mass stars is crucial for understanding how these stars might form.

Line surveys of HMSFRs in $\mathrm{mm} / \mathrm{sub}-\mathrm{mm}$ atmospheric windows exhibit rich molecular emission spectra (see Herbst \& van Dishoeck 2009 for a comprehensive review).

\footnotetext{
* Appendices are available in electronic form at http://www . aanda.org

$\star \star$ Merged maps (data cubes) are only available at the CDS via anonymous ftp to cdsarc.u-strasbg. fr (130.79.128.5) or via http://cdsarc.u-strasbg.fr/viz-bin/qcat?]/A+A/581/A71
}

The multitude of detected lines allow for an unbiased look at the chemical composition of a particular region, from which its physical properties can be inferred, including temperature and density. However, HMSFRs are typically distant (several kpc), so that spatially disentangling the physical and chemical substructures is challenging with the resolution of most facilities. This makes the Becklin-Neugebauer and Kleinmann-Low Nebula in Orion (Orion BN/KL), at a distance of $414 \pm 7 \mathrm{pc}$ (Sandstrom et al. 2007; Menten et al. 2007), ideal for studies of the physical and chemical properties of HMSFRs. As the nearest HMSFR, it has been extensively studied at both far-infrared to (sub) millimetre wavelengths accessible from space (e.g., Olofsson et al. 2007; Bergin et al. 2010; Crockett et al. 2014) and at radio, millimetre, and submillimetre wavelengths accessible from the ground. A broad range of studies on this source have been conducted either with single-dish telescopes (e.g., Johansson et al. 1984; Sutton et al. 1985; Blake et al. 1987; Turner 1989; Greaves \& White 1991; Schilke et al. 1997, 2001; Lee \& Cho 2002; Comito et al. 2005; Remijan et al. 2008; Tercero et al. 2010) or interferometers (e.g., Wright et al. 1996; Blake et al. 1996; Beuther et al. 2005, 2006; Friedel \& Snyder 2008; Guélin et al. 2008). Strong emission lines in these 
observations have revealed complex dynamics (e.g., Sutton et al. 1985; Blake et al. 1987; Turner 1989; Beuther \& Nissen 2008; Beuther et al. 2010) and chemistry (e.g., Wright et al. 1996; Blake et al. 1996; Beuther et al. 2005, 2006; Friedel \& Snyder 2008; Guélin et al. 2008; Widicus Weaver \& Friedel 2012; Friedel \& Widicus Weaver 2012).

Both single-dish-only and interferometer-only observations, however, have limitations. Single-dish observations have low spatial resolution, making it difficult to detect spatial variations of chemistry on small scales. In contrast, interferometric observations can probe spatial variations on small scales, but suffer from filtering-out to large scale structures (the "missing short spacing" problem; Bajaja \& van Albada 1979), making measurements of the chemistry of more extended features unreliable. In this paper, we aim to study the chemistry of Orion-KL by obtaining a combined dataset from an interferometer (Submillimetre Array, SMA) and a single-dish (IRAM $30 \mathrm{~m}$ ) telescope. These combined data, which resolve small-scale substructures and are sensitive to more extended components, allow us to accurately map the distribution of molecular species, determine their abundances, and ultimately analyse spatial variations in the chemistry.

One unique feature of HMSFRs is hot molecular cores, which are compact objects $(\leq 0.05 \mathrm{pc})$ that exhibit high temperatures $(\gtrsim 100 \mathrm{~K})$ and densities $\left(\gtrsim 10^{6} \mathrm{~cm}^{-3}\right.$; Palau et al. 2011) Numerous spectral surveys (see, e.g., Turner 1989; Schilke et al. 2001; Belloche et al. 2009; Herbst \& van Dishoeck 2009; Tercero et al. 2010) have revealed that hot molecular cores are characterized by a large abundance of simple organic molecules, including $\mathrm{H}_{2} \mathrm{CO}$ (formaldehyde), $\mathrm{HCOOH}$ (formic acid), and complex organic molecules (COMs, organics containing $\geq 6$ atoms), such as $\mathrm{CH}_{3} \mathrm{OH}$ (methanol), $\mathrm{HCOOCH}_{3}$ (methyl formate), and $\mathrm{CH}_{3} \mathrm{OCH}_{3}$ (dimethyl ether). Although some larger COMs have low abundances in hot molecular cores, e.g., $\mathrm{CH}_{3} \mathrm{CH}_{2} \mathrm{OH}$ (ethanol; Rizzo et al. 2001), $\mathrm{CH}_{3} \mathrm{COCH}_{3}$ (acetone; Snyder et al. 2002), $\mathrm{CH}_{3} \mathrm{CHO}$ (acetaldehyde; Nummelin et al. 2000), $\left(\mathrm{CH}_{2} \mathrm{OH}\right)_{2}$ (ethylene glycol; Hollis et al. 2002), and $\mathrm{CH}_{2} \mathrm{OHCHO}$ (glycolaldehyde; Hollis et al. 2000, 2004), they have been previously detected towards the Galacticcentre hot molecular core source SgrB2 (N-LMH). Because of their high degree of chemical and structural complexity, the precise origins and formation mechanisms of these COMs is still a subject of debate. Nevertheless, understanding these topics is particularly important for understanding the chemical evolution of the interstellar medium (ISM; e.g., Herbst \& van Dishoeck 2009). Recently, a number of interferometric observations of COMs in Orion-KL (e.g., Friedel et al. 2005; Friedel \& Snyder 2008; Guélin et al. 2008; Favre et al. 2011a,b; Widicus Weaver \& Friedel 2012) have shown spatial-related chemical differentiation. However, the relatively low sensitivity of these observations means that the lowest-abundance $\mathrm{COMs}$ (e.g., $\mathrm{CH}_{2} \mathrm{OHCHO}, \mathrm{CH}_{3} \mathrm{CH}_{2} \mathrm{OH}$, and $\mathrm{CH}_{3} \mathrm{CHO}$ ) are below the $3 \sigma$ detection limit or are blended by strong neighbouring lines.

In addition to the hot molecular core chemistry, the shock chemistry driven by outflows is another important aspect of chemistry in HMSFRs. The region in Orion-KL that is most affected by outflow and shock events is called the "plateau" (Blake et al. 1987; Wright et al. 1996; Lerate et al. 2008; Esplugues et al. 2013) and is characterized by spectral lines with broad line wings. Two roughly perpendicular outflows are seen in Orion-KL, traced by the proper motion of $\mathrm{H}_{2} \mathrm{O}$ masers at $22 \mathrm{GHz}$ (Genzel et al. 1981). One is a high-velocity outflow (30-100 $\mathrm{km} \mathrm{s}^{-1}$, along the north-west-south-east
(NW-SE) axis); this outflow is also detected in $\mathrm{OH}$ (hydroxyl) masers (Norris 1984; Cohen et al. 2006), broad bipolar CO line wings (Zuckerman et al. 1976; Kwan \& Scoville 1976; Erickson et al. 1982; Masson et al. 1987; Chernin \& Wright 1996; Beuther \& Nissen 2008; Zapata et al. 2009), and lobes of poorly collimated shock-excited $\mathrm{H}_{2}$ (Beckwith et al. 1978; Scoville et al. 1982; Taylor et al. 1984; Allen \& Burton 1993; Sugai et al. 1994). Although the source responsible for driving the outflow is still uncertain, it has been suggested that the outflow is only about 500-700 years old (Lee \& Burton 2000; Doi et al. 2002; Gómez et al. 2005, 2008; Nissen et al. 2007; Beuther \& Nissen 2008; Bally et al. 2011; Goddi et al. 2011b; Nissen et al. 2012). The other is a low-velocity outflow $\left(\sim 18 \mathrm{~km} \mathrm{~s}^{-1}, \Delta V \sim 35 \mathrm{~km} \mathrm{~s}^{-1}\right.$, along the north-eastsouth-west (NE-SW) axis; Genzel et al. 1981; Gaume et al. 1998), which is also detected in SO (Plambeck et al. 1982), SiO masers (Wright \& Plambeck 1983; Plambeck et al. 2009; Greenhill et al. 2013) and $\mathrm{H}_{2} \mathrm{O}$ maser (Greenhill et al. 2013). This outflow is believed to be closely associated with an expanding gas shell or torus around Source I, but whether its age is $>3000$ years (Menten \& Reid 1995; Greenhill et al. 1998; Doeleman et al. 1999; Reid et al. 2007) or much younger (Plambeck et al. 2009; Greenhill et al. 2013) is still not clear. Another goal of this study is to look for additional chemical signposts of these outflows, apart from the known masers, in order to better understand their physical and chemical properties.

In this paper, we present the first combined single-dish and interferometric line survey at $1.3 \mathrm{~mm}$ towards Orion-KL and study the chemical properties of this region at a linear resolution of $\sim 1200 \mathrm{AU}$ (at a distance of $\sim 414 \mathrm{pc}$ ). In Sect. 2, we describe the observations and their combination. In Sect. 3, we present a continuum map and maps of the distribution of different molecules, including simple molecules and COMs, where the presences of COMs are identified by synthetic spectrum fittings. The spatial distributions, column densities, and abundances for all the detected species are derived in Sect. 4. A discussion of the chemistry in the hot molecular core and outflows of OrionKL can be found in Sect. 5 .

\section{Observations and data reduction}

\subsection{Submillimetre Array (SMA)}

We carried out observations of Orion-KL with the SMA on Feb. 15, 2005 and Feb. 19, 2005. At $1.3 \mathrm{~mm}(220 / 230 \mathrm{GHz})$ in the compact configuration with seven antennas, the baselines ranged between 16 and $69 \mathrm{~m}$. The short baseline cutoff causes source structures $\geq 20^{\prime \prime}$ to be filtered out. The primary beam is $52^{\prime \prime}$, and the phase centre of the observations is $\alpha_{2000}=$ $05^{\mathrm{h}} 35^{\mathrm{m}} 14.5^{\mathrm{s}}$ and $\delta_{2000}=-5^{\circ} 22^{\prime} 30.45^{\prime \prime}$ with $V_{\mathrm{lsr}} \sim 7 \mathrm{~km} \mathrm{~s}^{-1}$. Bandpass calibration was done with Callisto, and the flux calibration is estimated to be accurate within $15 \%$, based on the SMA monitoring of quasars. Phase and amplitude calibration was performed via frequent observations of the quasar 0607-157. The zenith opacities, measured with the NRAO tipping radiometre located at the Caltech Submillimetre Observatory, were excellent during both tracks with $\tau(1.3 \mathrm{~mm}) \sim 0.03-0.04$, allowing us to observe at both $1.3 \mathrm{~mm}$ and $440 \mu \mathrm{m}$ simultaneously (Beuther et al. 2006). More technical descriptions of the SMA and its calibration schemes can be found in Ho et al. (2004). The receiver operated in a double-side band mode with an intermediate frequency of 4-6 GHz so that the upper and lower side bands were separated by $10 \mathrm{GHz}$. The correlator had a bandwidth of $2 \mathrm{GHz}$, and the channel separation was $0.8125 \mathrm{MHz}$, corresponding to a velocity resolution of $\sim 1.2 \mathrm{~km} \mathrm{~s}^{-1}$. Double-sided band 
system temperatures corrected for the atmosphere were between 150 and $300 \mathrm{~K}$ (depending on the elevation of the source).

The initial flagging and calibration was done with the IDL superset MIR, which is originally developed for the Owens Valley Radio Observatory and adapted for the SMA ${ }^{1}$. The imaging and data analysis was conducted in MIRIAD (Sault et al. 1995) and CASA ${ }^{2}$. Between the observations on two nights, the frequency setups were shifted by $0.4 \mathrm{GHz}$, yielding a slightly larger total bandwidth for the combined dataset (218.870-221.234 GHz for the lower sideband, and 228.870-231.234 GHz for the upper sideband). The synthesised beams of the combined data are $3.49^{\prime \prime} \times 2.57^{\prime \prime}$ for the lower sideband and $3.68^{\prime \prime} \times 2.69^{\prime \prime}$ for the upper sideband.

\subsection{Single-dish observations with the IRAM $30 \mathrm{~m}$ telescope}

In addition to the high spatial resolution data from the SMA, we mapped an area of $1^{\prime} \times 1^{\prime}$, with the IRAM $30 \mathrm{~m}$ telescope, to complement the short-spacing information missing from the interferometric observations. Observations were performed in the on-the-fly mode on Nov. 30, 2012, covering a broad bandpass (8 GHz bandwidth for each sideband) of EMIR at 220/230 GHz. Observations were conducted under good weather conditions $\left(T_{\text {sys }}=266 \mathrm{~K}, \tau \sim 0.152\right.$ at $\left.220 \mathrm{GHz}\right)$. The focus was on Uranus, and pointing was checked on Uranus and 0420-014. Using a forward efficiency of $92 \%$ and a main beam efficiency of $58 \%$, we converted the data from antenna temperature $(\mathrm{K})$ to flux (Jy) by using an efficiency ${ }^{3}$ of 7.8 . The beam of the $30 \mathrm{~m}$ telescope at $1.3 \mathrm{~mm}$ is $\sim 11.3^{\prime \prime}$ in the upper sideband and $\sim 11.8^{\prime \prime}$ in the lower sideband. The observations cover frequencies 213.370-221.150 GHz and 229.051-236.831, with a velocity resolution of $0.255 \mathrm{~km} \mathrm{~s}^{-1}$. We used the Gildas ${ }^{4}$ software for data reduction, and then converted the $30 \mathrm{~m}$ data to the MIRIAD data format.

\subsection{Data merging}

The $30 \mathrm{~m}$ single-dish spectral-line data were first converted to visibilities, and then combined with the SMA data using the MIRIAD package task UVMODEL ${ }^{5}$. To recover large-scale emission, while simultaneously maintaing high spatial resolutions for resolving small scale structure, we chose an intermediate weighting between natural and uniform ("robust" $=-0.5$ value in MIRIAD). However, as continuum information is not available from the $30 \mathrm{~m}$ observations, only the spectral-line data can be corrected in this way.

The combined data have a field of view of $52^{\prime \prime}$ and a beam of $5.55^{\prime \prime} \times 4.35^{\prime \prime}$ with position angle $\mathrm{PA}=+8^{\circ}$ in the lower sideband and $5.44^{\prime \prime} \times 4.43^{\prime \prime}$ with $\mathrm{PA}=+2^{\circ}$ in the upper sideband. The frequency overlap between the SMA and $30 \mathrm{~m}$ data results in the combined data having a frequency range 218.870-221.150 GHz and 229.051-231.234 GHz. Figure 1 presents an amplitude-versus-projected uv distance plot, showing the good combination of the single-dish and interferometer data. Missing projected baselines shortwards of $10 \mathrm{k} \lambda$ from the SMA have been complemented by using the $30 \mathrm{~m}$ data. After

\footnotetext{
1 http: //cfa-www.harvard. edu/ cqi/mircook.html

2 http://casa.nrao.edu

3 http://www . iram. es/IRAMES/mainWiki/

Iram30mEfficiencies

4 http: //WWW.iram. fr/IRAMFR/GILDAS

5 https://www. cfa.harvard.edu/sma/miriad/manuals/

SMAuguide/smauserhtml/uvmodel.html
}
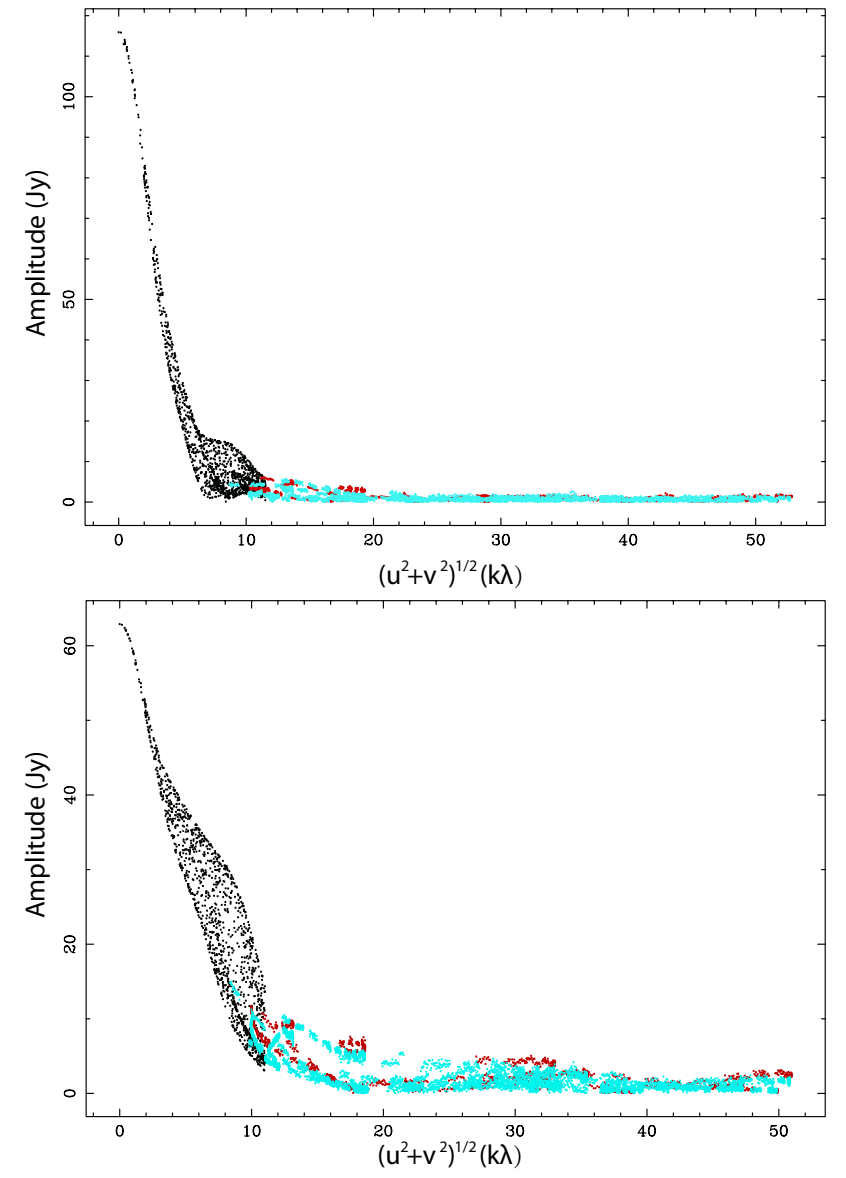

Fig. 1. Amplitude in Jy versus the projected baseline showing the combination of the interferometer and single-dish data, from the upper sideband (top) to lower sideband (bottom). Red and blue dots present data from the SMA on Feb. 15, 2005 and Feb. 19, 2005, respectively, showing the lack of baselines shorter than $10 \mathrm{k} \lambda$. Black dots are data converted from $30 \mathrm{~m}$ single-dish observations, which fill in the missing short spacing information.

combination, the $1 \sigma \mathrm{rms}$ per $1.2 \mathrm{~km} \mathrm{~s}^{-1}$ channel is measured to be $80 \mathrm{mJy} \mathrm{beam}^{-1}$ for the lower sideband and $70 \mathrm{mJy} \mathrm{beam}^{-1}$ for the upper sideband.

\section{Observational results}

\subsection{Continuum emission}

Orion-KL has a rich spectrum of molecular emission lines, so extracting the continuum requires some care. To construct our continuum map, we averaged the line-free parts of the upper and lower sidebands from the SMA-only data. In Fig. 2, we present the resulting $1.3 \mathrm{~mm}$ continuum image as contours overlaid on a map of the outflow traced by shock-excited $\mathrm{H}_{2}$ (Nissen et al. 2007). We detected the previously known compact continuum substructures of this region and moreover, resolved some of these substructures into multiple distinct condensations. We list the following resolved condensations in Table 1:

- The brightest hot core (HC): located close to a wellstudied infrared source IRc2 (Downes et al. 1981; Wynn-Williams et al. 1984), the brightest HC is the first clump in Orion-KL detected by the high spatial resolution mm observations (Masson et al. 1985; Mundy et al. 1986; Woody et al. 1989; Wright et al. 1992), and it exhibits 


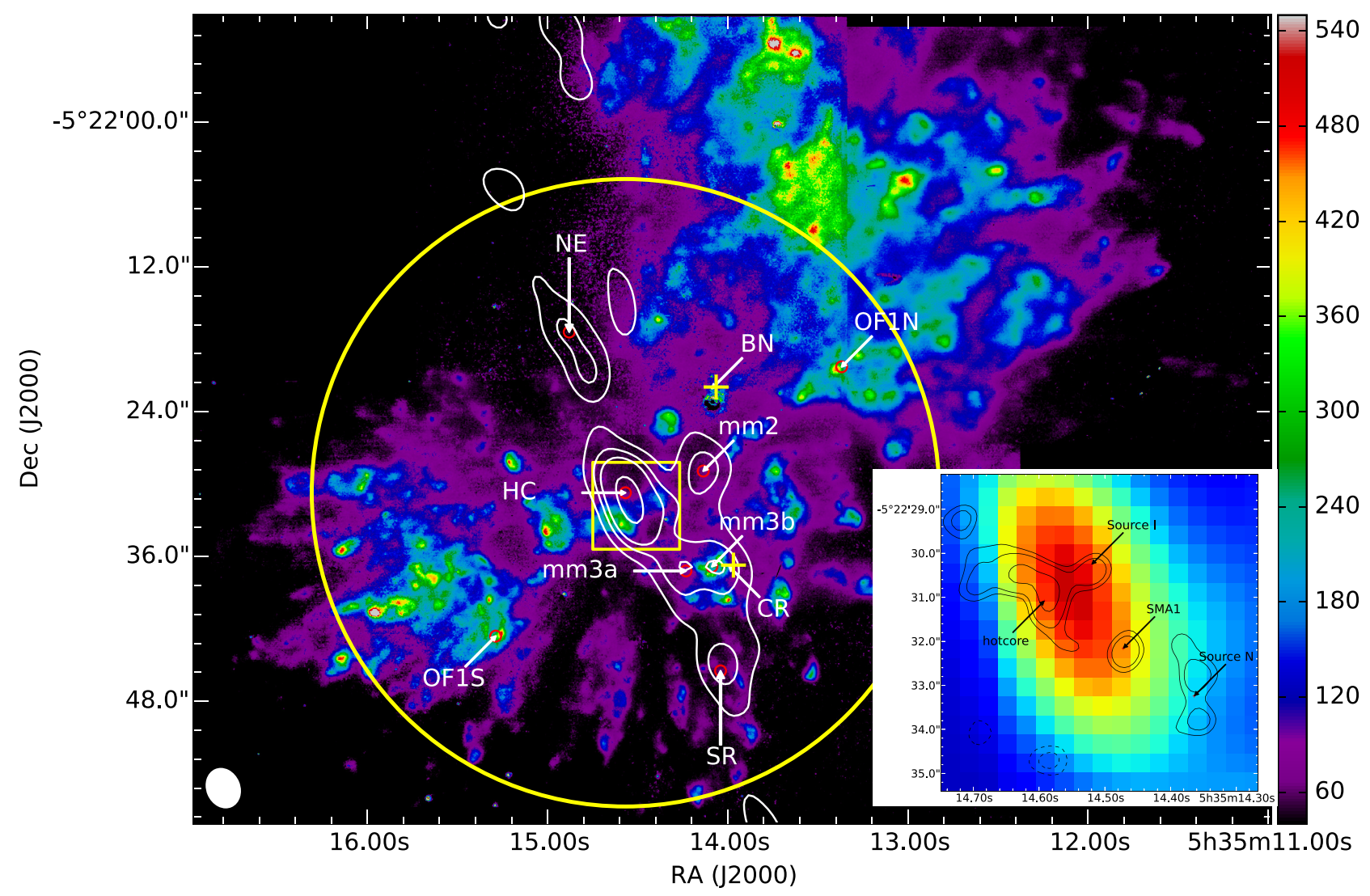

Fig. 2. Substructures resolved by SMA-only observations at a spatial resolution of $\sim 1200 \mathrm{AU}$. White contours show the $1.3 \mathrm{~mm}$ continuum emission at $-5 \sigma, 5 \sigma, 15 \sigma, 25 \sigma, 60 \sigma \mathrm{rms}$ levels with the $\sigma=0.04 \mathrm{Jy} \mathrm{beam}^{-1}$. The yellow circle denotes the primary beam of SMA at $1.3 \mathrm{~mm}$. Red circles denote the peaks of hot core (HC), mm2, mm3a, mm3b, the southern region (SR), north-east clump (NE), the NW and SE parts of the high-velocity outflow (OF1N, OF1S). Yellow crosses denote the BN object and the compact ridge (CR). The beam in the bottom left corner is from the SMA-only data. Colourmap shows the brightness (in counts per $400 \mathrm{~s}$ ) of shocked $\mathrm{H}_{2}$ emission (Nissen et al. 2007). Corresponding to the yellow square, the insert panel shows the HC continuum from the SMA at $1.3 \mathrm{~mm}$ in detail as colourmap, with black contours of the $865 \mu \mathrm{m}$ continuum emission at a spatial resolution of $\sim 300 \mathrm{AU}$ from Beuther et al. (2004) overlaid.

the highest peak specific intensity $\left(\mathrm{Jy} \mathrm{beam}^{-1}\right)$ in the $1.3 \mathrm{~mm}$ SMA continuum map. With the temperature estimated to be 100-300 K (e.g., Wilner et al. 1994; Wilson et al. 2000; Beuther et al. 2005), this source has been found to be abundant in hydrogenated species and nitrogen- $(\mathrm{N}-)$ bearing molecules (Sutton et al. 1995; Blake et al. 1996; Wright et al. 1996; Wilson et al. 2000). With even higher resolution (0.7", $300 \mathrm{AU})$, observations from SMA at $865 \mu \mathrm{m} / 348 \mathrm{GHz}$ expose additional substructures within the HC (Beuther et al. 2004). Presented as black contours overlaid on the $1.3 \mathrm{~mm}$ colourmap in the inner panel of Fig. 2, these $865 \mu \mathrm{m}$ structures are: SMA1 (a high-mass protostellar source, suggested to be the driving source of the high-velocity outflow; Beuther \& Nissen 2008), Source I (located at the centre of the $\mathrm{SiO}$ masers and believed to drive the bipolar low-velocity outflow; Menten \& Reid 1995; Matthews et al. 2010), Source N (a Herbig Ae/Be or mid-B star with a luminosity of $\sim 2000 L_{\odot}$; Menten \& Reid 1995; Greenhill et al. 2004; Gómez et al. 2005; Rodríguez et al. 2005; Goddi et al. 2011b; Nissen et al. 2012), and hotcore. Hereafter, we use "HC" to denote the whole region resolved by $1.3 \mathrm{~mm}$ continuum at a spatial resolution of $\sim 1200$ AU, the "hotcore" to denote the position resolved by $865 \mu \mathrm{m}$ continuum at a spatial resolution of $\sim 300 \mathrm{AU}$, and the "hot molecular core" to describe an evolutionary status.
- $\mathrm{mm} 2$ : located $\sim 7^{\prime \prime}$ west of the $\mathrm{HC}$, and $\sim 5^{\prime \prime}$ south of the BN object. Coincident with infrared sources IRc3, IRc6, and IRc20 (Dougados et al. 1993), this condensation is also known as the western clump (WC; Wright et al. 1992), or the north-west clump (NWC; Blake et al. 1996; Tang et al. 2010; Favre et al. 2011a). The mass $\left(2-3 M_{\odot}\right), \mathrm{H}_{2}$ column density, and high temperature (a few hundred $\mathrm{K}$ ) in this source suggest that it may be the site of ongoing low-mass star formation (Schreyer \& Henning 1999).

- mm3a and mm3b: these two condensations are located south-west of the HC. Coincident with infrared IRc5 (Dougados et al. 1993), they were previously considered to be a single clump called the south-west clump (SWC; Tang et al. 2010), or CntC (Murata et al. 1992), situated at the compact ridge (CR; Blake et al. 1996; Wright et al. 1996; Tang et al. 2010). Similar dual continuum peaks have been detected in other high spatial resolution observations of HMSFRs (Tang et al. 2010; Favre et al. 2011a; Friedel \& Widicus Weaver 2011), so we consider them to be separated. The $\mathrm{CR}$ is known to have different kinematics and chemistry than in the HC: it is a vast region ( 15" $-25^{\prime \prime}$; Plume et al. 2012; Esplugues et al. 2013) with lower temperature (Genzel \& Stutzki 1989), is dominated by oxygen- (O-) bearing molecules (Blake et al. 1987; Wright et al. 1996; Liu et al. 2002), and is believed to be the region where two outflows interact with the dense ambient 
S. Feng et al.: Resolving the chemical substructure of Orion-KL

Table 1. Properties of substructures, which are denoted on the continuum image obtained from SMA observations at $1.3 \mathrm{~mm}$ (Fig. 2).

\begin{tabular}{|c|c|c|c|c|c|c|c|c|}
\hline Source & & $\begin{array}{c}\mathrm{RA}(\alpha) \\
{[\mathrm{J} 2000]} \\
5 \mathrm{~h} 35 \mathrm{~m} \sim\end{array}$ & $\begin{array}{l}\operatorname{Dec}(\delta) \\
{[\mathrm{J} 2000]} \\
-5^{\circ} 22^{\prime} \sim\end{array}$ & $\begin{array}{c}I_{v}{ }^{a} \\
(\mathrm{Jy} / \text { beam })\end{array}$ & $\begin{array}{l}T_{\text {rot }} \\
(\mathrm{K})\end{array}$ & $\begin{array}{c}N_{\mathrm{H}_{2}, 1}{ }^{d} \\
\left(10^{23} \mathrm{~cm}^{-2}\right) \\
3.49^{\prime \prime} \times 2.57^{\prime \prime}\end{array}$ & $\begin{array}{c}N_{\mathrm{H}_{2}, 2^{e}} \\
\left(10^{23} \mathrm{~cm}^{-2}\right) \\
5.55^{\prime \prime} \times 4.35^{\prime \prime}\end{array}$ & Alternative designations \\
\hline \multirow[t]{2}{*}{$\mathrm{HC}$} & & $14.6 \mathrm{~s}$ & $31.0^{\prime \prime}$ & $2.75 \pm 0.3$ & $\begin{array}{l}155 \pm 16^{b_{1}} \\
121 \pm 16^{b_{2}} \\
126 \pm 13^{b_{3}} \\
225 \pm 19^{b_{4}} \\
199 \pm 45^{b_{5}}\end{array}$ & $\begin{array}{l}23.7 \pm 4.6 \\
31.0 \pm 6.7 \\
29.6 \pm 5.8 \\
16.3 \pm 2.9 \\
18.4 \pm 5.1\end{array}$ & $11.9 \pm 0.7$ & \multirow[t]{2}{*}{$\mathrm{Ca}^{j}, \mathrm{SMM}^{l}, \mathrm{CntB}^{k}$} \\
\hline & $\begin{array}{l}\text { hotcore }^{f} \\
\text { SMA1 }^{f} \\
\text { Source I }{ }^{g} \\
\text { Source } \mathbf{N}^{g}\end{array}$ & $\begin{array}{l}14.58 \mathrm{~s} \\
14.46 \mathrm{~s} \\
14.51 \mathrm{~s} \\
14.35 \mathrm{~s}\end{array}$ & $\begin{array}{l}30.97^{\prime \prime} \\
32.36^{\prime \prime} \\
30.53^{\prime \prime} \\
33.49^{\prime \prime}\end{array}$ & & & & & \\
\hline $\mathrm{mm} 2$ & & $14.1 \mathrm{~s}$ & $27.1^{\prime \prime}$ & $0.87 \pm 0.10$ & $\begin{array}{c}108 \pm 4^{b_{1}} \\
112 \pm 7^{b_{3}} \\
174 \pm 14^{b_{4}} \\
173 \pm 40^{b_{5}}\end{array}$ & $\begin{array}{c}11.0 \pm 1.7 \\
10.5 \pm 1.8 \\
6.7 \pm 1.2 \\
6.7 \pm 1.9 \\
\end{array}$ & $9.3 \pm 0.1$ & $\begin{array}{l}\mathrm{NWC}^{h, i}, \mathrm{Cc}^{j}, \mathrm{SMM}^{l}, \\
\mathrm{WC}^{n}, \mathrm{CntD}^{k}\end{array}$ \\
\hline \multirow[t]{3}{*}{$\mathrm{CR}^{l}$} & & $14.0 \mathrm{~s}$ & $36.9^{\prime \prime}$ & & & & & $\mathrm{SWC}^{i}, \mathrm{SMM}^{l}$ \\
\hline & $\mathrm{mm} 3 \mathrm{a}$ & $14.3 \mathrm{~s}$ & $37.9^{\prime \prime}$ & $0.96 \pm 0.09$ & $\begin{array}{c}103 \pm 3^{b_{1}} \\
101 \pm 5^{b_{3}} \\
159 \pm 12^{b_{4}} \\
101 \pm 26^{b_{5}} \\
\end{array}$ & $\begin{array}{c}12.8 \pm 1.6 \\
13.1 \pm 1.8 \\
8.1 \pm 1.3 \\
13.1 \pm 3.7\end{array}$ & $6.2 \pm 0.07$ & $\mathrm{Cb}^{j}{ }^{j}, \mathrm{CntC}^{k}$ \\
\hline & $\mathrm{mm} 3 \mathrm{~b}$ & $14.1 \mathrm{~s}$ & $36.4^{\prime \prime}$ & $1.01 \pm 0.11$ & $\begin{array}{c}88 \pm 6^{b_{1}} \\
102 \pm 5^{b_{3}} \\
160 \pm 17^{b_{4}} \\
89 \pm 24^{b_{5}}\end{array}$ & $\begin{array}{c}15.9 \pm 2.7 \\
13.5 \pm 2.1 \\
8.5 \pm 1.6 \\
15.6 \pm 4.8\end{array}$ & $6.7 \pm 0.05$ & $\mathrm{Cb} 2^{j}, \mathrm{CntC}^{k}$ \\
\hline $\mathrm{NE}$ & & $14.8 \mathrm{~s}$ & $19.1^{\prime \prime}$ & $0.38 \pm 0.09$ & $43 \pm 3^{b_{1}}$ & $20.1 \pm 4.2$ & $3.3 \pm 0.06$ & $\mathrm{CntH}^{k}$ \\
\hline SR & & $14.0 \mathrm{~s}$ & $45.4^{\prime \prime}$ & $0.41 \pm 0.06$ & $43 \pm 3^{c}$ & $14.2 \pm 2.9$ & $1.0 \pm 0.02$ & $\mathrm{Cd}^{j}, \mathrm{MM}_{2} 3^{m}$ \\
\hline OF1N & & $13.4 \mathrm{~s}$ & $20.5^{\prime \prime}$ & $\sigma=0.04$ & $43 \pm 3^{c}$ & $<4.2$ & $\sim 1.1$ & $\mathrm{PK} 1^{o}$ \\
\hline OF1S & & $15.3 \mathrm{~s}$ & $42.5^{\prime \prime}$ & $\sigma=0.03$ & $43 \pm 3^{c}$ & $<3.1$ & $\sim 0.6$ & $\mathrm{PK}^{\circ}{ }^{\circ}$ \\
\hline $\mathrm{BN}^{o}$ & & $14.11 \mathrm{~s}$ & $22.7^{\prime \prime}$ & & & & & \\
\hline
\end{tabular}

Notes. Positions with upper case are from the references listed in the footnotes, without are from $1.3 \mathrm{~mm}$ SMA continuum observations. ${ }^{(a)}$ Peak specific intensity $I_{v}$ is obtained from each substructure of SMA-only continuum. ${ }^{(b 1)}$ Excitation temperature is from $\mathrm{CH}_{3} \mathrm{CN}$ rotation map fitting in Fig. 8. ${ }^{(b 2)}$ Excitation temperature is from $\mathrm{CH}_{3}^{13} \mathrm{CN}$ rotation map fitting in Fig. 8. ${ }^{(b 3)}$ Excitation temperature is from $\mathrm{HCOOCH}_{3}$ rotation map fitting in Fig. 9. ${ }^{(b 4)}$ Excitation temperature is from $\mathrm{CH}_{3} \mathrm{OH}$ rotation map fitting in Fig. 9. ${ }^{(b 5)}$ Excitation temperature is from ${ }^{34} \mathrm{SO}_{2}$ rotation map fitting in Fig. 9. ${ }^{(c)}$ Excitation temperature is assumed the same as that in $\mathrm{NE}$ from $\mathrm{CH}_{3} \mathrm{CN} .{ }^{(d)} \mathrm{H}_{2}$ column density is calculated from the SMA-only continuum at substructure peak using Eq. (11), performed in Sect. 4.2.2. ${ }^{(e)} \mathrm{H}_{2}$ column density is calculated from the conversion of combined SMA-30 $\mathrm{m} \mathrm{C}^{18} \mathrm{O}$ at temperatures derived from $\mathrm{HCOOCH}_{3}$, performed in Sect. 4.2.2.

References. ${ }^{(f)}$ Beuther et al. (2004); ${ }^{(g)}$ Menten \& Reid (1995); ${ }^{(h)}$ Blake et al. (1996); ${ }^{(i)}$ Tang et al. (2010); ${ }^{(j)}$ Favre et al. (2011a); ${ }^{(k)}$ Murata et al. (1992); ${ }^{(l)}$ Zapata et al. (2011); ${ }^{(m)}$ Eisner et al. (2008) ; ${ }^{(n)}$ Wright et al. (1992); ${ }^{(o)}$ Beckwith et al. (1978).

material (Blake et al. 1987; Liu et al. 2002). Therefore, to ensure that we fully characterise its chemical properties, the following study focuses separately on $\mathrm{mm} 3 \mathrm{a}$ and $\mathrm{mm} 3 \mathrm{~b}$, instead of considering CR as a whole.

- The southern ridge (SR): the southernmost condensation detected in the $1.3 \mathrm{~mm}$ continuum map. Although it has been detected in other observations (Eisner et al. 2008; Favre et al. 2011a), it has not been characterized in detail.

- The north-east clump (NE): a strong continuum emission peak which is adjacent the $\mathrm{HC}$ and the north extended ridge (ER; Sutton et al. 1985; Blake et al. 1987). This clump is usually filtered out in high spatial resolution observations, but can be clearly detected in interferometric observations made with large scale configurations (Murata et al. 1992; Tang et al. 2010). Comparison with the existing single-dish maps suggests it is part of a large-scale dusty filament along the NE-SW direction (e.g., detected by JCMT at $450 \mu \mathrm{m}$, Wright et al. 1992; SCUBA at $450 \mu \mathrm{m}$ and $850 \mu \mathrm{m}$,
Di Francesco et al. 2008). In addition, this clump is in the plateau of the low-velocity outflow, so its chemistry may be affected by shocks (Sect. 5.3).

Because of the limited UV coverage and field of view, the highvelocity outflow and the BN object are not detected above $5 \sigma$ in the SMA-only continuum map. To study the chemistry in these regions from the combined data, we take the positions of the high-velocity plateau $\left(V_{\mathrm{lsr}} \sim 11 \mathrm{~km} \mathrm{~s}^{-1}\right)$ and $\mathrm{BN}$ object from Beckwith et al. (1978) and denote them as OF1N, OF1S, and BN in Fig. 2.

Table 1 lists the nominal absolute positions of these substructures, as well as the peak specific intensity $\left(\mathrm{Jy} \mathrm{beam}^{-1}\right)$ for each continuum substructure, obtained from the SMA-only $1.3 \mathrm{~mm}$ continuum map (Fig. 2, for more detail about this table, see Sect. 4.2.2). The HC is the brightest condensation; $\mathrm{mm} 2, \mathrm{~mm} 3 \mathrm{a}$, and $\mathrm{mm} 3 \mathrm{~b}$ are fainter; while NE, SR, OF1N, and OF1S are even weaker. 


\subsection{Spectral line emission}

\subsubsection{Line identification}

After imaging the whole SMA- $30 \mathrm{~m}$ combined data cube, we extracted the double sideband spectrum from each substructure denoted in Fig. 2 and show them in Fig. 3. The majority of lines are strongest towards the $\mathrm{HC}$, so we use the spectrum from the HC to identify lines. However, line identification may be ambiguous in a region such as the Orion-KL HC, because of a high density of lines (that can have different line widths according the species), also because the $\sim 1.2 \mathrm{~km} \mathrm{~s}^{-1}$ spectral resolution of the observations does not allow us to separate all the blending. For strong lines, we first compare their rest frequencies with an existing single-dish survey catalogue of Orion-KL by Sutton et al. (1985). Next, we crosscheck their rest frequencies with the molecular database at "Splatalogue" (a compilation of the Jet Propulsion Laboratory (JPL ${ }^{7}$, Pickett et al. 1998), Cologne Database for Molecular Spectroscopy catalogues (CDMS ${ }^{8}$, Müller et al. 2005), and Lovas/NIST catalogues, Lovas 2004), and confirm the identification if the CDMS/JPL intensity is $>10^{-6} \mathrm{~nm}^{2} \mathrm{MHz}^{9}$.

Using the above method, the detection of strong lines are shown in Fig. A.1. In addition, we can also detect multiple transitions of low-abundance COMs in Orion-KL, owing to the high sensitivity and recovering the full intensity for spatially extended molecular emission from the combined dataset. We use a synthetic spectrum fitting program (Sanchez-Monge 2011; Palau et al. 2011) in conjunction with molecular data from the JPL/CDMS catalogues, to simultaneously fit multiple COM lines under the assumption that all transitions are optically thin, are in local thermodynamic equilibrium (LTE), and have the same line width ${ }^{10}$. Given these assumptions, the fitted flux densities are not always a perfect match to the observed spectra. However, in many cases we are able to robustly identify multiple transitions of a COM (Fig. A.2). We discuss the properties of the identified COM species further in Sect. 3.2.2.

With these two approaches, we identified more than 160 lines that we assigned to 20 molecules (including 25 isotopologues) over the entire band; we labelled the unblended strong emissions in Fig. 3, and list all the lines in Table A.1. This table includes the rest frequency, quantum number, symmetry label, and upper state energy for each transition. Lines marked with "†" are from molecules containing less than six atoms as shown in Fig. 4, and those marked with " $\neq$ " are from molecules we designated as COMs and carbon chains, which are shown in Fig. 5. Our observations have a frequency resolution of $0.8125 \mathrm{MHz}$ $\left(\sim 1.2 \mathrm{~km} \mathrm{~s}^{-1}\right.$ at $\left.1.3 \mathrm{~mm}\right)$. Thus, some observed lines with broad linewidths can be attributed to multiple transitions, when compared to the laboratory-measured rest frequencies recorded in the "Splatalogue" database. If potentially blended transitions for one line are from different isotopologues but have similarly

\footnotetext{
${ }^{6}$ www.splatalogue.net

7 http://spec.jpl.nasa.gov

8 http://www.astro.uni-koeln.de/cdms/catalog

9 This is a coarse cutoff for identifying the strong emission lines, corresponding to spontaneous emission rate $A_{\mathrm{ul}} \sim 10^{-5.5 \sim-6}$. Taking the frequency resolution of our observations into consideration $(\sim 0.8 \mathrm{MHz})$, we found that $50 \%$ number possibility at certain rest frequency of a particular line has CDMS/JPL intensity $>10^{-6}$.

10 The synthetic fitting program we use here is for line detection, but not for finding the best fit of column density or temperature to the observations. Since the synthetic fitting is performed with optically thin assumption, minor lines of a particular species which cannot fit as well as the majority may be optically thick.
}

strong CDMS/JPL intensities, we compared the spatial distribution of this line to those of the known isotopologues. Then, we either confirm a match or indicate all possibilities with "*”" in Table A.1. If there are multiple possible transitions from the same isotopologue at this rest frequency, they are also marked with “*”.

\subsubsection{Line imaging}

In general, all the identified species show different spatial distributions, velocity widths (FWHM $\Delta V$ ), and velocity at line centre $\left(V_{\text {peak }}\right)$ in each substructure (see Fig. A.3, on average, $V_{\text {peak }} \sim 6-8 \mathrm{~km} \mathrm{~s}^{-1}$ in the HC; $V_{\text {peak }} \sim 8-10 \mathrm{~km} \mathrm{~s}^{-1}$ in mm2, $\mathrm{mm} 3 \mathrm{a}$, and mm3b; $V_{\text {peak }} \sim 10-12 \mathrm{~km} \mathrm{~s}^{-1}$ in NE). However, transitions from a single isotopologue have the same spatial distribution (except for $\mathrm{CH}_{3} \mathrm{OH}$ ). In this paper, we have chosen one of the strongest unblended transitions from each isotopologue and produced their intensity distribution maps from the SMA-30 m combined data. In avoiding contamination from other strong lines, we integrate the intensity of each line through $40-60 \%$ of their profile range in Fig. A. $3^{11}$.

The combined data at $220 / 230 \mathrm{GHz}$ covers a field of view of $52^{\prime \prime}$, which is greater than the extent of the source (not including the outflows), so our maps exhibit negligible artefacts caused by residual missing flux. The high quality of our data allows us to map molecules, such as ${ }^{34} \mathrm{SO}_{2}, \mathrm{O}^{13} \mathrm{CS}$, $\mathrm{HNCO}, \mathrm{H}_{2}^{13} \mathrm{CO},{ }^{13} \mathrm{CO}$, and $\mathrm{CH}_{2} \mathrm{CO}$ for the first time with both high resolution and sensitivity to spatial information on all scales. After sorting by different groups, we present the intensity integration map for each identified molecule in Figs. 4 and 5. Compared to previous sub$\mathrm{mm}$ and $\mathrm{mm}$ (especially interferometric) observations in OrionKL (listed in the following paragraphs), the main features of our molecular distribution maps are:

1. All the identified sulphur- (S-) bearing species in our dataset are diatomic/triatomic containing no hydrogen. Most of them have extended distributions (except for $\mathrm{SO}_{2}$ and ${ }^{34} \mathrm{SO}_{2}$ ), exhibiting a V-shaped morphology that extends from the emission peak at $\mathrm{HC}$, through mm3a, mm3b to $\mathrm{mm} 2$. Their velocities at line centre also vary among substructures from $\sim 6 \mathrm{~km} \mathrm{~s}^{-1}$ (HC) through 8-10 $\mathrm{km} \mathrm{s}^{-1}(\mathrm{~mm} 2, \mathrm{~mm} 3 \mathrm{a}, \mathrm{mm} 3 \mathrm{~b}$, and SR) to $11-12 \mathrm{~km} \mathrm{~s}^{-1}$ (NE).

- OCS and $\mathrm{O}^{13} \mathrm{CS}$ (carbonyl sulphide) peak at SMA1 and have emission that extend to the CR. In addition, OCS is also detected in NE and SR. Although only one line of $\mathrm{O}^{13} \mathrm{CS}$ is detected in our data $(19 \rightarrow 18$, Fig, 6), other lines of this isotopologue have been previously confirmed and imaged by single-dish observations (e.g., Sutton et al. 1985; Ziurys \& McGonagle 1993; Tercero et al. 2010). In addition to the detections by single-dish surveys (e.g., Sutton et al. 1985; Turner 1991; Schilke et al. 1997; Tercero et al. 2010), previous interferometric surveys of OCS also reproduce the same V-shaped widespread intensity distribution of this molecule, peaking at the HC (e.g., Wright et al. 1996; Friedel \& Snyder 2008).

- $\mathrm{SO}_{2}$ and ${ }^{34} \mathrm{SO}_{2}$ (sulphur dioxide) peak at the hotcore and SMA1 and exhibit extended emission in the NE direction, with $V_{\text {peak }} \sim 9-11 \mathrm{~km} \mathrm{~s}^{-1}$. Unlike the other S-bearing species, no emission has been detected

\footnotetext{
11 The chosen velocity ranges are slightly larger than the FWHM of the lines, when accounting for the small variation in the peak velocity between different substructures.
} 
S. Feng et al.: Resolving the chemical substructure of Orion-KL

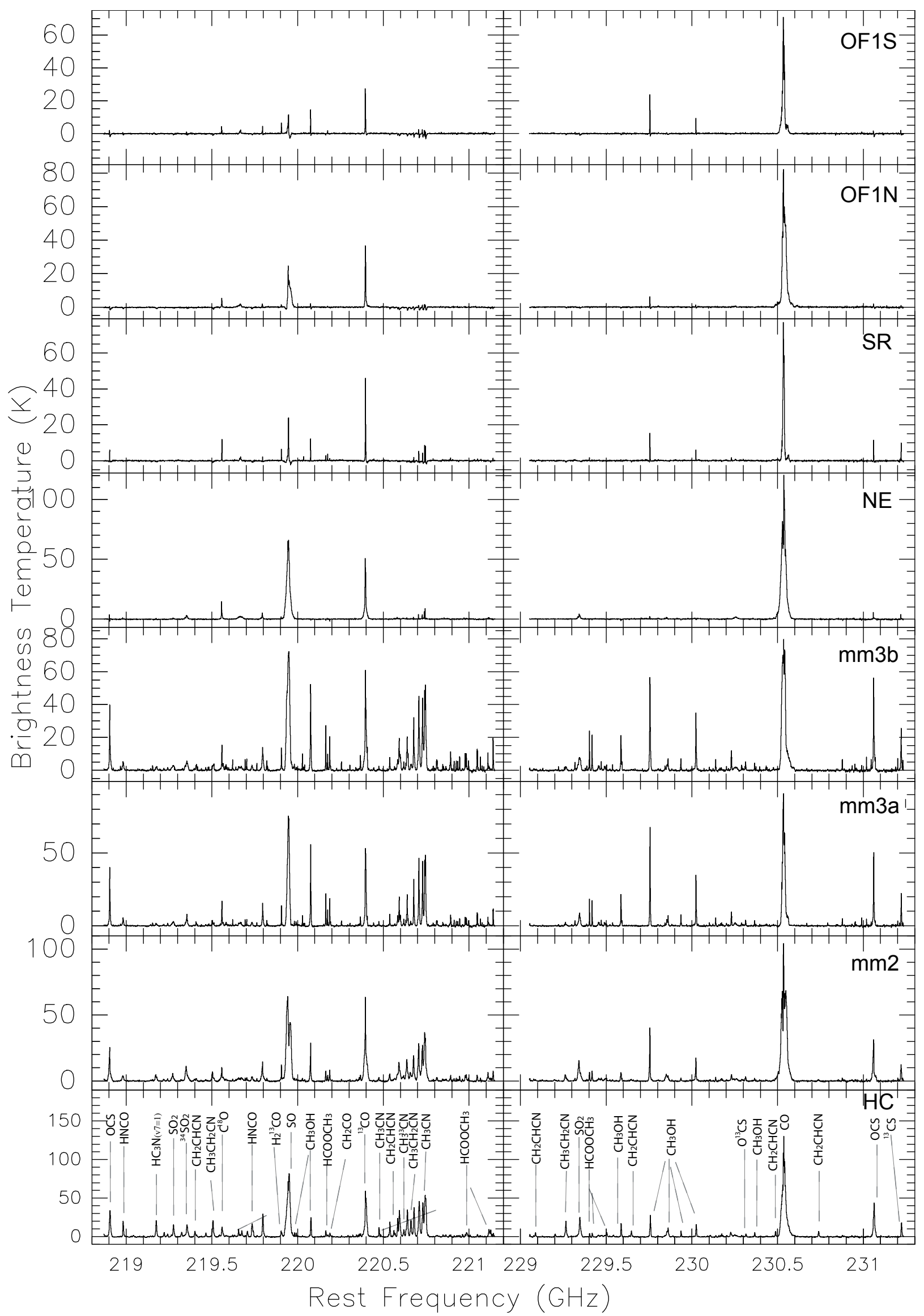

Fig. 3. Spectra taken towards selected positions (denoted by red circles in Fig. 2) after imaging the whole data cube. The spectral resolution is smoothed to $1.2 \mathrm{~km} \mathrm{~s}^{-1}$ after the combination of SMA and $30 \mathrm{~m}$ data, and strong emission lines are labelled. A blow-up figure of the spectrum in the $\mathrm{HC}$ is Fig. A.1. 

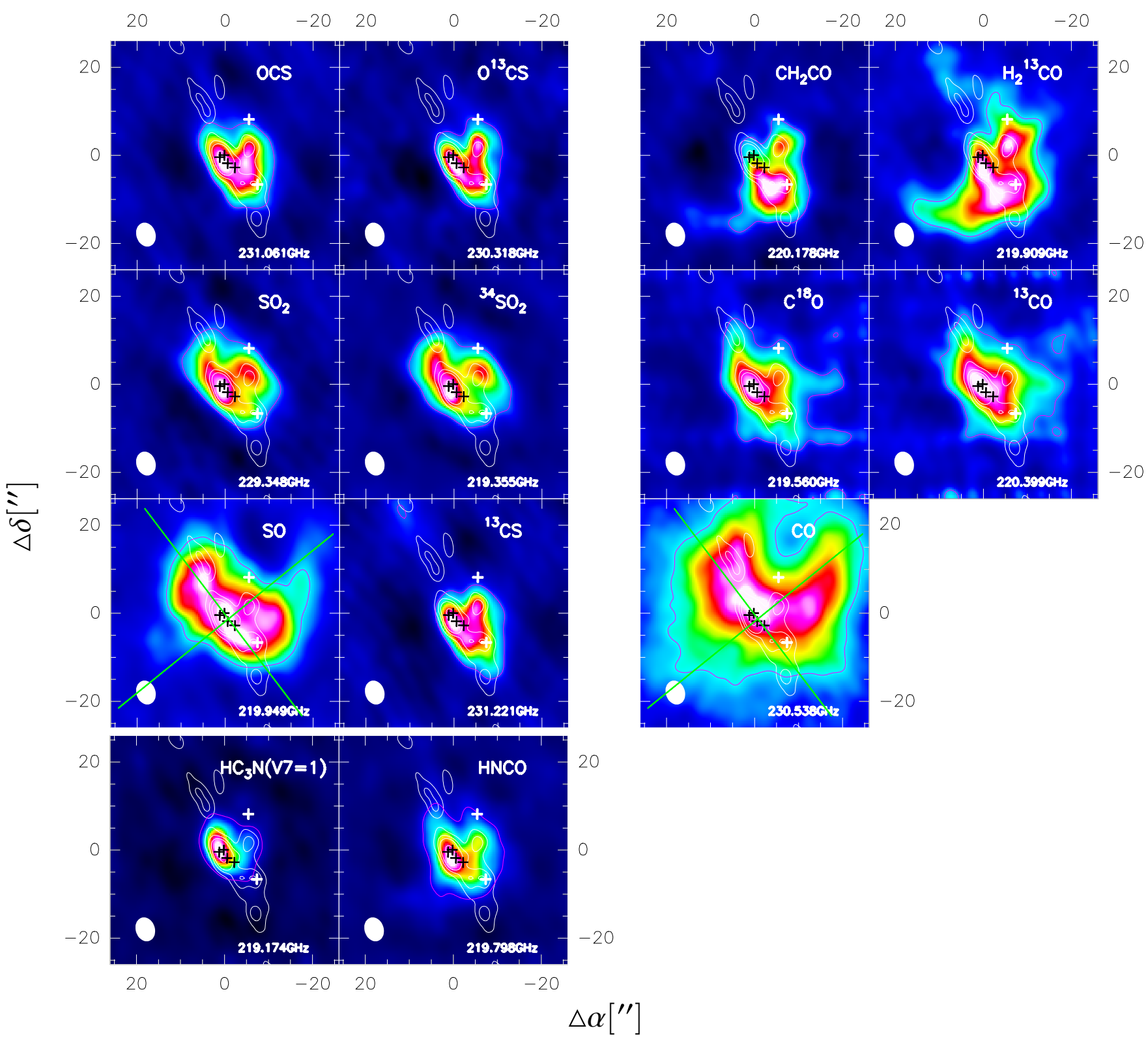

Fig. 4. Intensity distribution maps of transitions from molecules containing $<6$ atoms, which are detected in the combined SMA-30m data at $1.3 \mathrm{~mm}$ (corresponding quantum numbers are listed in Table A.1, with " + " marks). The intensities have been derived by integrating the line emission over the velocity range shown in Fig. A.3. The filled beam in the bottom left corner is from SMA-30 m data. White contours show the continuum from the SMA-only data (at $-5 \sigma, 5 \sigma, 15 \sigma, 25 \sigma$, and $60 \sigma$ levels). Purple contours show $\pm 1 \sigma$ levels of the molecular emission (solid contours indicate positive flux, and dashed contours indicate negative flux). Green lines in the SO and CO maps sketch the outflow directions. The white crosses denote the BN object and the CR, and the black crosses denote the positions of the hotcore, Source I, Source N, and SMA1, as in Fig. 2. All images have different colour scales (in Jy beam ${ }^{-1} \mathrm{~km} \mathrm{~s}^{-1}$ ), increasing from black to white, which are optimized to emphasize the features in the distribution of each molecules.

from these species in SR, and they do not display a clear V-shaped feature. However, there is a clear second velocity component in the line profile of ${ }^{34} \mathrm{SO}_{2}$ (at $\sim 22.7 \mathrm{~km} \mathrm{~s}^{-1}$, Fig. 7) and $\mathrm{SO}_{2}$ (at $22.1 \mathrm{~km} \mathrm{~s}^{-1}$, Fig. 7). In addition, red- and blue-shifted ${ }^{34} \mathrm{SO}_{2}$ trace outflows in the NW-SE direction (Fig. A.4). Singledish studies (e.g., $\mathrm{SO}_{2}$; Blake et al. 1987; Persson et al. 2007; Esplugues et al. 2013, ${ }^{34} \mathrm{SO}_{2}$; Esplugues et al. 2013) conclude that this species is a good tracer not only for the warm dense gas, but also for regions affected by shocks. While based on the velocity around
$22 \mathrm{~km} \mathrm{~s}^{-1}$, Esplugues et al. (2013) ${ }^{12}$ suggests that this velocity component may be associated with shocks caused by the explosive (NW-SE) outflow and/or the $\mathrm{BN}$ object, our data clearly show that this velocity component of $\mathrm{SO}_{2}$ is spatially offset from $\mathrm{BN}$ and seems to be associated much more with the main NE-SW outflow (Fig. A.5). On small scales, interferometric observations of Orion-KL also detect a compact $\mathrm{SO}_{2}$ emission peak (Wright et al. 1996; Zapata et al. 2011) and strong extended emission of ${ }^{34} \mathrm{SO}_{2}$ to $\mathrm{mm} 2$ (Beuther et al. 2005).

$12 V_{\text {peak }}=20.5 \mathrm{~km} \mathrm{~s}^{-1}$ in Esplugues et al. (2013). 


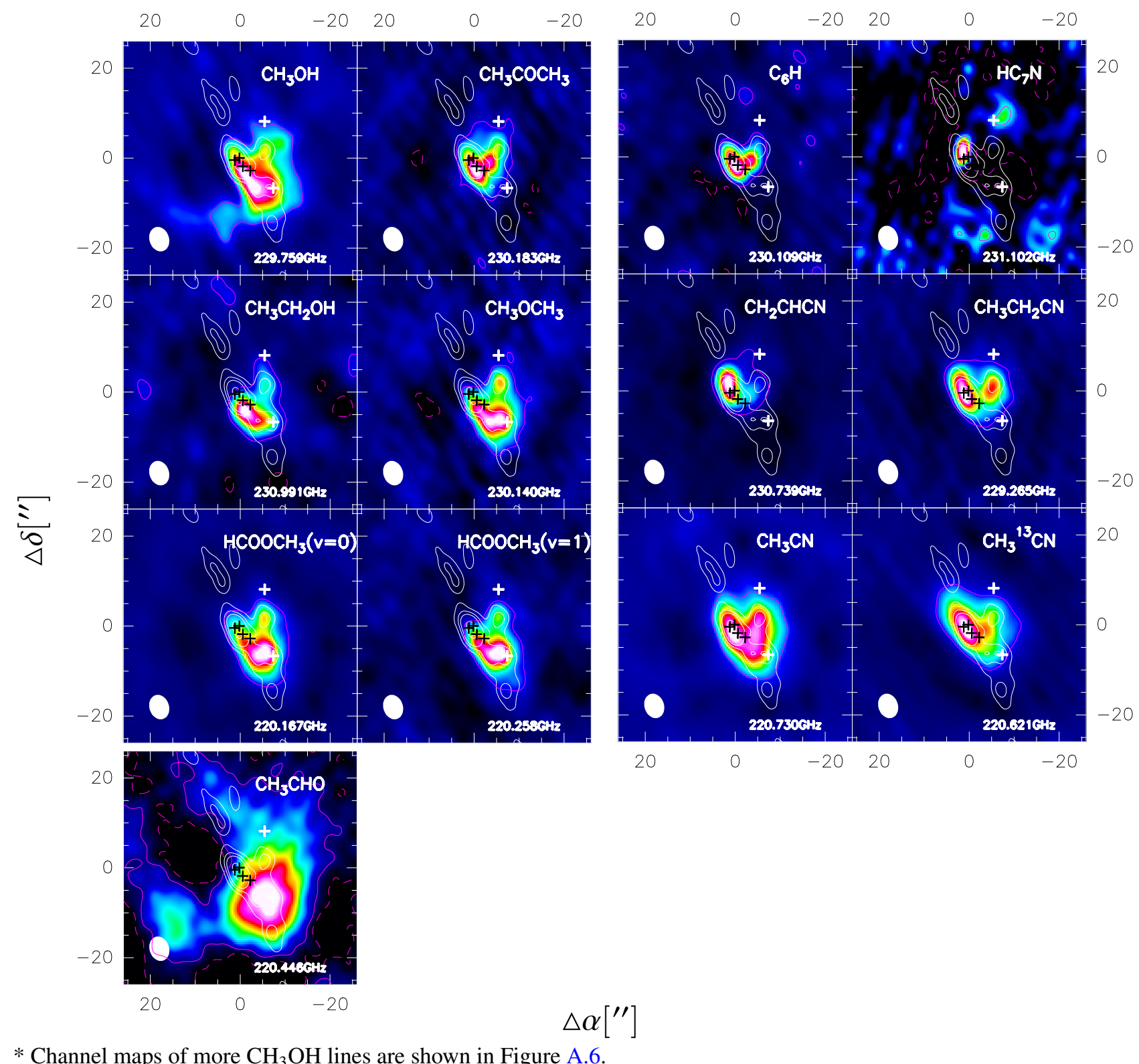

Fig. 5. Intensity distribution maps of transitions from COMs and carbon chains detected in the combined SMA-30 m data at $1.3 \mathrm{~mm}$ (corresponding quantum numbers are list in Table A.1, with " $\neq$ " marks). The intensities have been derived by integrating the line emission over the velocity range shown in Fig. A.3. The filled beam in the bottom left corner is from SMA-30 m data. White contours show the continuum from the SMA-only data (at $-5 \sigma, 5 \sigma, 15 \sigma, 25 \sigma$, and $60 \sigma$ levels). Purple contours show $\pm 1 \sigma$ levels of the molecular emission (solid contours indicate positive flux, and dashed contours indicate negative flux). The white crosses denote the BN object and the CR, and the black crosses denote the positions of the hotcore, Source I, Source N, and SMA1, as in Fig. 2. All images have different colour scales (in Jy beam ${ }^{-1} \mathrm{kms}^{-1}$ ), increasing from black to white, which are optimized to emphasize the features in the distribution of each molecules.

- SO (sulphur monoxide) has multiple strong emission peaks towards $\mathrm{HC}, \mathrm{NE}, \mathrm{mm} 3 \mathrm{a}$, and $\mathrm{mm} 3 \mathrm{~b}$ and exhibits strong self-absorption in $\mathrm{mm} 2$ (Fig. A.3). There is also significant emission extending along both the plateau of high- $\left(V_{\text {peak }} \sim 8-10 \mathrm{~km} \mathrm{~s}^{-1}\right)$ and low-velocity $\left(V_{\text {peak }} \sim 7-8 \mathrm{~km} \mathrm{~s}^{-1}\right)$ outflows. Similar to ${ }^{34} \mathrm{SO}_{2}$, its line wings are broad $\left(\triangle V \sim 23 \mathrm{~km} \mathrm{~s}^{-1}\right)$, and a second velocity component is clear in the line profile at $\sim 21 \mathrm{~km} \mathrm{~s}^{-1}$ (see Fig. A.3 mm3b), which may be associated with shock events ${ }^{13}$. These morphological features (elongated

\footnotetext{
13 The line wing of $\mathrm{SO}$ at $\sim 7 \mathrm{~km} \mathrm{~s}^{-1}$ is broad, and its second velocity component is blended, so we cannot tell the origin of the shock precisely from our observations, Fig. A.5.
}

emission peak(s) and broad line wings of SO) have also been detected by both single-dish (Persson et al. 2007; Esplugues et al. 2013) and interferometric (Wright et al. 1996) line studies.

- ${ }^{13} \mathrm{CS}$ (carbon monosulphide) has only one transition in our dataset, but it is very strong, peaking at SMA1, with a similar distribution to $\mathrm{O}^{13} \mathrm{CS}$ - extended emission from $\mathrm{NE}$ through $\mathrm{HC}$ to the $\mathrm{CR}$. Owing to its high dipole moment, this molecule is considered to be a dense gas tracer (e.g., Bronfman et al. 1996), and its detection in both CR and NE is confirmed by Persson et al. (2007) and Tercero et al. (2010). 

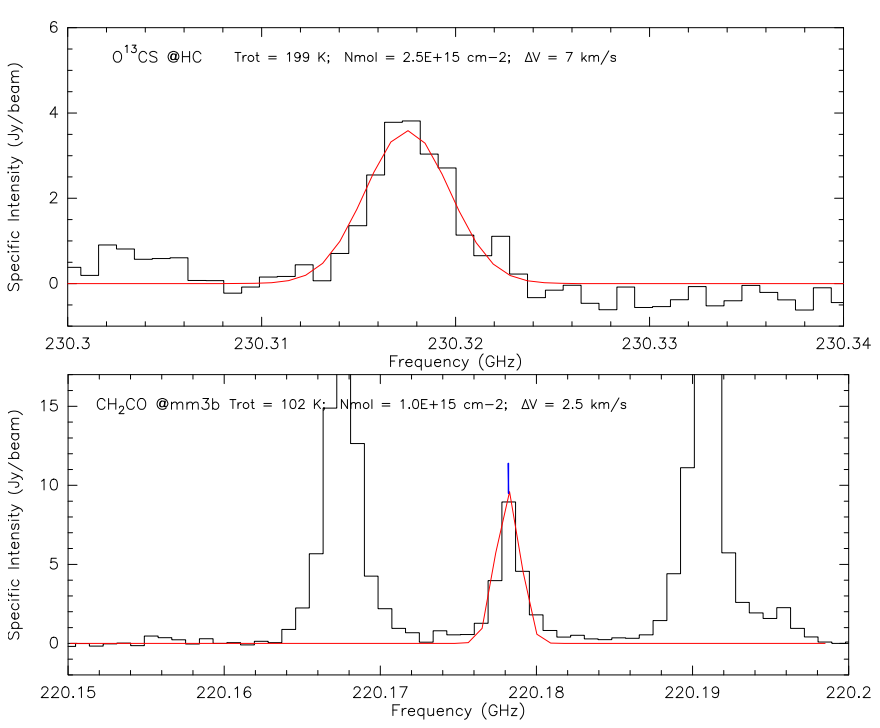

Fig. 6. Lines that only have one transition with low specific intensity $\left(\leq 10 \mathrm{Jy}\right.$ beam $\left.^{-1}\right)$ in our data, fitted with synthetic spectrum from their emission peaks.

2. The distribution of simple $\mathrm{C}_{x} \mathrm{H}_{y} \mathrm{O}_{z}(x, z=1,2 \ldots ; y=$ $0,1,2 \ldots ; x+y+z \leq 5)$ molecules are co-spatially extended.

- $\mathrm{CO}, \mathrm{C}^{18} \mathrm{O}$, and ${ }^{13} \mathrm{CO}$ (carbon monoxide) have a common morphological peak at the $\mathrm{HC}$ (though the integrated intensity of $\mathrm{CO}$ peaks north of the $\mathrm{HC}$ due to its strong self-absorption in this source, see Fig. A.3). Velocity at line centre $V_{\text {peak }}$ ranges from $6 \mathrm{~km} \mathrm{~s}^{-1}$ (HC) through $8 \mathrm{~km} \mathrm{~s}^{-1}$ (mm2, mm3a, mm3b, and SR) to $10 \mathrm{~km} \mathrm{~s}^{-1}$ (NE, OF1N, and OF1S), with broad red- and blueshifted line wings trace both high-velocity $\left(\mathrm{CO},{ }^{13} \mathrm{CO}\right)$ and low-velocity $\left(\mathrm{C}^{18} \mathrm{O}\right)$ outflows (Fig. A.4). As the second most abundant species in the ISM, this species has been detected in numerous other observations of Orion-KL $\left(\mathrm{C}^{18} \mathrm{O}\right.$; Beuther \& Nissen 2008; Plume et al. 2012, CO; Zuckerman et al. 1976; Kwan \& Scoville 1976; Erickson et al. 1982; Blake et al. 1987, singledish; Masson et al. 1987; Chernin \& Wright 1996; Zapata et al. 2009; Peng et al. 2012b, interferometry, ${ }^{13} \mathrm{CO}$; Wilson et al. 2011, single-dish), showing the same morphology as we see: extended structure, with bipolar line wings covering from the NE through $\mathrm{HC}$ to the CR and tracing outflows.

- $\mathrm{CH}_{2} \mathrm{CO}$ (ketene) has a single transition $\left(11_{1,11} \rightarrow 10_{1,10}\right)$ detected (Fig. 6), peaking at $\mathrm{mm} 3 \mathrm{a}$ and $\mathrm{mm} 3 \mathrm{~b}$, with strong emission extending to the OF1S and the NE filament. With $V_{\text {peak }} \sim 8 \mathrm{~km} \mathrm{~s}^{-1}$ and $\Delta V \sim 2.5 \mathrm{~km} \mathrm{~s}^{-1}$ in $\mathrm{mm} 2, \mathrm{~mm} 3 \mathrm{a}$, and $\mathrm{mm} 3 \mathrm{~b}$, this line has also been confirmed by Sutton et al. (1985). Some other lines of this species are also detected in Orion-KL by Lee \& Cho (2002).

- $\mathrm{H}_{2}^{13} \mathrm{CO}$ (formaldehyde) exhibits an elongated peak stretching from SMA1 towards mm3a. Similar to the simple organic $\mathrm{CH}_{2} \mathrm{CO}$, it also shows extended structure along the SE lobe of the high-velocity outflow and NE. Single-dish detections (e.g., Persson et al. 2007) confirm that this molecule is present in both the CR and the outflows.
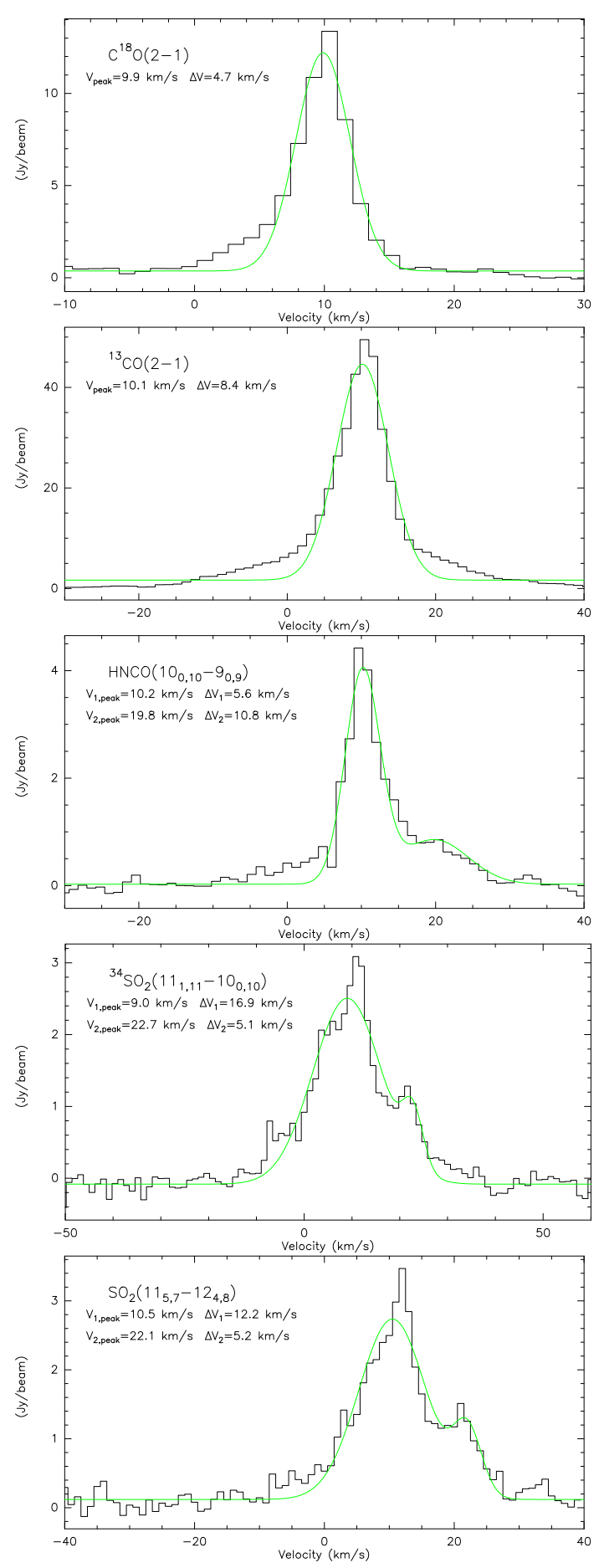

Fig. 7. Gaussian fitting to the line profile of ${ }^{13} \mathrm{CO}, \mathrm{C}^{18} \mathrm{O}, \mathrm{HNCO}, \mathrm{SO}_{2}$, and ${ }^{34} \mathrm{SO}_{2}$ extracted from NE. Outflow signatures are seen in the broad line wings and the second velocity component in the $\mathrm{HNCO}, \mathrm{SO}_{2}$, and ${ }^{34} \mathrm{SO}_{2}$ line profile.

3. HNCO (isocyanic acid) is the simplest organic molecule containing $\mathrm{C}, \mathrm{H}, \mathrm{O}$, and $\mathrm{N}$ elements. Peaking at SMA1, it shows extended emission towards all the substructures, with $V_{\text {peak }}$ ranging from $6 \mathrm{~km} \mathrm{~s}^{-1}$ (HC) through 7-8 $\mathrm{km} \mathrm{s}^{-1}$ (mm3a, mm3b, SR, and OF1S) to 9-10 $\mathrm{km} \mathrm{s}^{-1}$ (mm2, NE, OF1N), and a second velocity component at $\sim 19.8 \mathrm{~km} \mathrm{~s}^{-1}$ (Fig. 7). Although the formation pathway of HNCO is still not clear (Hasegawa \& Herbst 1993; Turner et al. 1999; Garrod et al. 2008; Tideswell et al. 2010), its distribution 
is observed to be correlated with shock tracing molecules $\mathrm{SiO}$ (e.g., Zinchenko et al. 2000) and $\mathrm{CH}_{3} \mathrm{OH}$ (e.g., Meier \& Turner 2005), suggesting that it is also produced in shocks.

4. In contrast, highly saturated $\mathrm{COMs}^{14}$ are the typical COMs detected in HMSFRs, and they exhibit a variety of morphological structures in Orion-KL. In general, N-bearing COMs have a similar peak to $\mathrm{HNCO}$, at or near $\mathrm{HC}$; while most of the O-bearing COMs are co-spatial with $\mathrm{CH}_{2} \mathrm{CO}$ peaking at $\mathrm{mm} 3 \mathrm{a}$ and $\mathrm{mm} 3 \mathrm{~b}$, and extending even to southern CR (e.g., $\mathrm{HCOOCH}_{3}, \mathrm{CH}_{3} \mathrm{OCH}_{3}$, and $\mathrm{CH}_{3} \mathrm{CHO}$ ). This spatial segregation of the $\mathrm{N}$ - and $\mathrm{O}$-bearing $\mathrm{COM}$ peaks has also been reported by other single-dish (Blake et al. 1987; Persson et al. 2007) and interferometric studies with high spatial resolution (e.g., Wright et al. 1996; Beuther et al. 2005; Favre et al. 2011a; Friedel \& Widicus Weaver 2012). In addition, there are several other O-bearing COMs identified in this study, which have unique morphologies. To understand the spatial differentiation of COMs on small scales (Fig. 5), we compared the distributions of these molecules between our observations to other recent interferometric studies (Table 2).

- $\mathrm{CH}_{3} \mathrm{CN}$ and $\mathrm{CH}_{3}^{13} \mathrm{CN}$ (methyl cyanide) are two abundant N-bearing COMs. In our dataset, we detect the $J=12 \rightarrow 11$ ladder of both isotopologues around $220 \mathrm{GHz}$. Most of the $\mathrm{CH}_{3}^{13} \mathrm{CN}$ lines are blended, but lines with $K=2,4,5,6$ are clearly detected in the $\mathrm{HC}$ (Fig. 8). In particular, $K=2$ line of $\mathrm{CH}_{3}^{13} \mathrm{CN}$ and $\mathrm{CH}_{3} \mathrm{CN}$ are clearly co-spatial (Fig. A.3), peaking at SMA1 $\left(V_{\text {peak }} \sim 5-7 \mathrm{~km} \mathrm{~s}^{-1}\right)$ and extending to $\mathrm{mm} 2$, mm3a, mm3b $\left(V_{\text {peak }} \sim 8-10 \mathrm{~km} \mathrm{~s}^{-1}\right)$, with $>3 \sigma$ detection in NE $\left(V_{\text {peak }} \sim 10-13 \mathrm{~km} \mathrm{~s}^{-1}\right)$, exhibiting a V-shaped morphology. $\mathrm{CH}_{3} \mathrm{CN}$ is detected in SR and OF1N (S), but not for $\mathrm{CH}_{3}^{13} \mathrm{CN}$. This $\mathrm{V}$-shaped morphology of $\mathrm{CH}_{3} \mathrm{CN}$ has also been detected in e.g., Beuther et al. (2005), Zapata et al. (2011), Widicus Weaver \& Friedel (2012), Bell et al. (2014), as well as the NE extension in Wright et al. (1996). This species is considered to be a warm and dense gas tracer (e.g., Kalenskii et al. 2000; Araya et al. 2005), with gas phase and grain surface chemical models predicting that it is only detected in an environment with elevated temperatures (e.g., Purcell et al. 2006). We use the $\mathrm{CH}_{3} \mathrm{CN}$ ladder and the $\mathrm{CH}_{3}^{13} \mathrm{CN}\left(12_{2} \rightarrow 11_{2}\right)$ line to estimate the temperature of the substructures where these lines are detected (see Sect. 4.1.1).

- $\mathrm{CH}_{2} \mathrm{CHCN}$ (vinyl cyanide) exhibits strong compact emission north of the hotcore, with $V_{\text {peak }} \sim 5 \mathrm{~km} \mathrm{~s}^{-1}$, and a slight extension towards $\mathrm{mm} 2$. The northern peak is also coincident with the one in higher resolution maps of this species from SMA at $865 \mu \mathrm{m}$ (Beuther et al. 2005), CARMA at $1 \mathrm{~mm}$ (Friedel \& Snyder 2008), and PdBI at $3 \mathrm{~mm}$ (Guélin et al. 2008).

- $\mathrm{CH}_{3} \mathrm{CH}_{2} \mathrm{CN}$ (ethyl cyanide) emission also makes a $\mathrm{V}$-shaped structure. Unlike the single-peaked structure of $\mathrm{CH}_{2} \mathrm{CHCN}, \mathrm{CH}_{3} \mathrm{CH}_{2} \mathrm{CN}$ exhibits a dual-peaked

\footnotetext{
14 A COM that has a chain of carbon atoms linked together by single bonds and has rich hydrogen atoms filling the other bonding orbitals of the carbon atoms.
}

structure, with a first emission peak at the hotcore $\left(\sim 6 \mathrm{~km} \mathrm{~s}^{-1}\right)$, and a second peak at $\mathrm{mm} 2\left(\sim 9 \mathrm{~km} \mathrm{~s}^{-1}\right)$. Although there are two velocity components in both $\mathrm{mm} 3 \mathrm{a}$ and $\mathrm{mm} 3 \mathrm{~b}\left(6 \mathrm{~km} \mathrm{~s}^{-1}, 10 \mathrm{~km} \mathrm{~s}^{-1}\right)$, there is no detection in southern $\mathrm{CR}$, which is consistent with Blake et al. (1987). This observed morphology is consistent with other interferometric observations at high spatial resolution (Wright et al. 1996; Beuther et al. 2005; Friedel \& Snyder 2008; Guélin et al. 2008; Widicus Weaver \& Friedel 2012; Peng et al. 2013).

- $\mathrm{CH}_{3} \mathrm{OH}$ (methanol) has multiple transitions in our data (Fig. A.2). We obtained the channel maps of all the unblended strong $\mathrm{CH}_{3} \mathrm{OH}$ transitions in our dataset and found two types of spatial distributions (Fig. A.6). One type of line clearly shows extended emission from $\mathrm{mm} 2$ and SR (7.2-8.4 $\mathrm{km} \mathrm{s}^{-1}$, e.g., lines of $8_{0,8} \rightarrow 7_{1,6} \mathrm{E}$, $8_{-1,8} \rightarrow 7_{0,7} \mathrm{E}$, and $3_{-2,2} \rightarrow 4_{-1,4} \mathrm{E}$ ) towards the highvelocity outflow $\mathrm{OF} 1 \mathrm{~N}(\mathrm{~S})$, exhibiting a similar SE tail to $\mathrm{H}_{2}^{13} \mathrm{CO}$ at $7-10 \mathrm{~km} \mathrm{~s}^{-1}$, which has not been detected before; whereas the other type of line (e.g., lines of $15_{4,11} \rightarrow 16_{3,13} \mathrm{E}, 22_{4,18} \rightarrow 21_{5,17} \mathrm{E}$, and $19_{5,14} \rightarrow$ $20_{4,17} \mathrm{~A}--$ ) does not show such a SE tail. However, both of them show multiple velocity-dependent emission peaks: towards SMA1 at $5 \mathrm{~km} \mathrm{~s}^{-1}$, mm3a at $8 \mathrm{~km} \mathrm{~s}^{-1}$, and $\mathrm{mm} 3 \mathrm{~b}$ at $10 \mathrm{~km} \mathrm{~s}^{-1}$. The multiple-peak structure is different from the traditional segregated dichotomous distribution of COMs in Orion-KL (i.e., the main emission peak is either at $\mathrm{HC}$ or $\mathrm{mm} 3 \mathrm{a}(\mathrm{b})$ ), though it has also been observed in other interferometric surveys (Wright et al. 1996; Beuther et al. 2005; Friedel \& Snyder 2008; Guélin et al. 2008; Friedel \& Widicus Weaver 2012; Peng et al. 2012a). Methanol has previously been detected in the Orion outflow (Wang et al. 2011), and furthermore, line $8_{-1,8} \rightarrow 7_{0,7}$ is known to be a potential Class I maser that traces shocks (Fish et al. 2011). As a result, the SE tail can be reasonably explained as a feature of the outflow. In addition, it is also suggested that the SMA1-Source $\mathrm{N}$ area is the launching point of the Orion-KL outflows (e.g., Menten \& Reid 1995; Beuther \& Nissen 2008), which may explain $\mathrm{CH}_{3} \mathrm{OH}$ and $\mathrm{H}_{2}^{13} \mathrm{CO}$ peaking in this area.

- $\mathrm{HCOOCH}_{3}$ (methyl formate) has the largest number of detected lines in our data (Fig. A.2). There are two types of $\mathrm{HCOOCH}_{3}$ in the whole observational band, torsionally excited lines $(v=1)$ and lines in ground state $(v=0)$. Both of them have identical distributions (Fig. 5) that peak at both mm3a and mm3b, with a clear detection in the southern $\mathrm{CR}$, which is similar to $\mathrm{CH}_{2} \mathrm{CO}$. This morphology has also been reported by Wright et al. (1996), Beuther et al. (2005), Guélin et al. (2008), Friedel \& Snyder (2008), Favre et al. (2011a), Widicus Weaver \& Friedel (2012), indicating that this species arises in a cooler and less dense environment than the $\mathrm{HC}$.

- $\mathrm{CH}_{3} \mathrm{OCH}_{3}$ (dimethyl ether) peaks towards mm3a and $\mathrm{mm} 3 \mathrm{~b}$ and extends to the southern $\mathrm{CR}$, similar to $\mathrm{HCOOCH}_{3}$. Unlike the other COMs, its emission in $\mathrm{mm} 2$ is considerably stronger than towards the HC. This morphology has also been imaged with other interferometric studies (Liu et al. 2002; Beuther et al. 2005; Guélin et al. 2008; Friedel \& Snyder 2008; Favre et al. 2011b; Brouillet et al. 2013). 
- $\mathrm{CH}_{3} \mathrm{CH}_{2} \mathrm{OH}$ (ethanol), the functional isomer ${ }^{15}$ of $\mathrm{CH}_{3} \mathrm{OCH}_{3}$, exhibits extended emission from the vicinity of $\mathrm{HC}$ to $\mathrm{mm} 2, \mathrm{~mm} 3 \mathrm{a}$, and $\mathrm{mm} 3 \mathrm{~b}$, indicating the same distinctive $\mathrm{V}$-shaped molecular emission as the N-bearing COMs. However, unlike $\mathrm{CH}_{3} \mathrm{OCH}_{3}$ and the other COMs, it peaks in between the hotcore and mm3a. The difference in the location of their peaks is exactly the same as reported by Guélin et al. (2008), Brouillet et al. (2013) from PdBI and Friedel \& Snyder (2008) with the EVLA and CARMA at high spatial resolution. In the grain mantle chemistry models, both isomers are produced from two radicals involved in the synthesis of $\mathrm{CH}_{3} \mathrm{OH}$ ice (Garrod et al. 2008). Therefore, their spatial differentiation may result from the chemical differences between the $\mathrm{CH}_{2} \mathrm{OH}$ and $\mathrm{CH}_{3} \mathrm{O}$ radicals across Orion-KL, e.g., variations in production rates or chemical evolution (Brouillet et al. 2013).

- $\mathrm{CH}_{3} \mathrm{COCH}_{3}$ (acetone) is an O-bearing COM, though, it has a similar distribution to $\mathrm{CH}_{3} \mathrm{CH}_{2} \mathrm{CN}$, which peaks at SMA1, extends to $\mathrm{mm} 2$, and has no detection in the southern CR. Although its nature as a hot molecular core tracer has been confirmed by detections in both Orion-KL (Friedel et al. 2005; Friedel \& Snyder 2008; Widicus Weaver \& Friedel 2012; Peng et al. 2013) and Sagittarius B2 (N-LHM) (Snyder et al. 2002), whether this species mainly forms on dust grains or in the gas phase is still not clear.

- $\mathrm{CH}_{3} \mathrm{CHO}$ (acetaldehyde) is tentatively detected, as all lines except one at $220.446 \mathrm{GHz}$ (Table A.1) are blended with $\mathrm{HCOOCH}_{3}$. Morphologically, this line has a similar distribution to $\mathrm{CH}_{2} \mathrm{CO}$, peaking at $\mathrm{mm} 3 \mathrm{a}$ and $\mathrm{mm} 3 \mathrm{~b}$, with extended emission to the NE and SE tail. Although its origin is still unclear (Charnley 2004), it has been detected in Orion-KL with single-dish telescopes (Turner 1989). Previous interferometric observations failed to detect it because of filtering out the extended structure (e.g., Friedel \& Snyder 2008; Widicus Weaver \& Friedel 2012). However, its detection has been reported by recent CARMA and ALMA observations (Loomis, priv. comm.), which shows the same distribution as our single-dish complemented interferometric observation result.

- There are also several other organics, such as $\mathrm{CH}_{2} \mathrm{OHCHO}$ (glycolaldehyde), $\left(\mathrm{CH}_{2} \mathrm{OH}\right)_{2}$ (ethylene glycol), and $\mathrm{HCOOH}$ (formic acid), which we do not detect in Orion-KL at $1.3 \mathrm{~mm}$ in the SMA-30 $\mathrm{m}$ combined data. Of the first two, although there are some candidate detections from our synthetic fitting, they are blended with other nearby lines, and so cannot be confirmed. Although Liu et al. (2002) claimed to detect $\mathrm{HCOOH}$ in the $\mathrm{CR}$, in their data the line at $1 \mathrm{~mm}$ is only a $2 \sigma$ feature. Widicus Weaver \& Friedel (2012) also did not detect any compact concentrations of $\mathrm{HCOOH}$ in our studied region either, and they conclude that the $\mathrm{HCOOH}$ emission must be extended on $>1700 \mathrm{AU}$ scales.

In brief, from our detections, $\mathrm{N}$ - and O-bearing COMs not only show two segregated peaks, but also exhibit more

\footnotetext{
15 Two compounds with the same molecular formula but different functional groups (radicals) are called functional isomers.
}

complicated spatial distributions. Most of the N-bearing species (except $\mathrm{CH}_{2} \mathrm{CHCN}$ ) are co-spatial and peak at the hotcore. While some O-bearing species $\left(\mathrm{HCOOCH}_{3}\right.$, $\mathrm{CH}_{3} \mathrm{OCH}_{3}$, and $\mathrm{CH}_{3} \mathrm{CHO}$ ) peak at mm3a and $\mathrm{mm} 3 \mathrm{~b}$, and others present more unusual features (i.e., $\mathrm{CH}_{3} \mathrm{OH}$ has velocity dependent peaks at both hotcore and $\mathrm{mm} 3 \mathrm{a} / \mathrm{mm} 3 \mathrm{~b}$; $\mathrm{CH}_{3} \mathrm{CH}_{2} \mathrm{OH}$ peaks in between mm3a and hotcore; and $\mathrm{CH}_{3} \mathrm{COCH}_{3}$ peaks in a similar location to $\mathrm{N}$-bearing COMs). This challenges the traditional hypothesis that all of these molecules are forming in the same chemical and physical conditions (Garrod et al. 2008; Laas et al. 2011).

5. Unlike the saturated COMs, long carbon chains (organics with carbon-carbon multiple bonds) are less likely to be detected in star-forming regions (Thorwirth 2001; Rice \& Maier 2013).

- $\mathrm{C}_{6} \mathrm{H}$ is the parent radical of $\mathrm{C}_{6} \mathrm{H}^{-}$(the first molecular anion identified in the gas phase interstellar and circumstellar media; McCarthy et al. 2006), after its presence was predicted by Herbst (1981). It had not been detected in Orion-KL before. In our dataset, there is one unblended line at $230.109 \mathrm{GHz}$ with $J=165 / 2 \rightarrow 163 / 2$, although most transitions identified by the synthetic fitting of $\mathrm{C}_{6} \mathrm{H}$ are blended. Emission from this tentative detected species appears to be confined to the HC.

- $\mathrm{HC}_{3} \mathrm{~N}$ and $\mathrm{HC}_{7} \mathrm{~N}$ are two cyanopolyynes $\left(\mathrm{HC}_{2 n+1} \mathrm{~N}, n\right.$ is an integer). We confirmed the detection of $\mathrm{HC}_{3} \mathrm{~N}\left(v_{7}=1\right)$ to the north of the hotcore and also tentatively detect $\mathrm{HC}_{7} \mathrm{~N}$. Although only two out of four of the $\mathrm{HC}_{7} \mathrm{~N}$ lines predicted by the synthetic fitting programme are detected in our observations ${ }^{16}$, these lines are unblended and peak at a similar location to $\mathrm{HC}_{3} \mathrm{~N}$. The excitedvibrational state $\left(v_{7}=1\right)$ of $\mathrm{HC}_{3} \mathrm{~N}$ shows a similar confined structure north-east of the $\mathrm{HC}$ in Wright et al. (1996); Beuther et al. (2005) and Zapata et al. (2011), where it is said to form a shell around the $\mathrm{HC} . \mathrm{HC}_{3} \mathrm{~N}$ is an especially useful dense gas tracer due to its relatively low optical depth transitions, large dipole moment, and simple linear structure (Bergin et al. 1996). The $\mathrm{HC}_{3} \mathrm{~N}\left(v_{7}=\right.$ 1) lines are believed to be primarily excited by a strong mid-infrared radiation field (e.g., Goldsmith et al. 1982). A gas-phase chemical model of a hot molecular core with high but transitory abundances of cyanopolyynes has been presented in Chapman et al. (2009), where these species are formed from reactions between the precursor $\mathrm{C}_{2 \mathrm{n}} \mathrm{H}_{2}$ (e.g., $\mathrm{C}_{2} \mathrm{H}_{2}$, acetylene) and $\mathrm{N}$-bearing species via gas phase chemistry (e.g., $\mathrm{C}_{2 n} \mathrm{H}_{2}+\mathrm{CN} \rightarrow \mathrm{HC}_{2 n+1} \mathrm{~N}+\mathrm{H}$ ).

\section{Rotational temperature and abundance estimates}

The above qualitative comparison of the morphology of different species show the chemical variations in the above-mentioned substructures. A more precise approach to studying these variations is to measure the quantitative differentiations in chemical parameters towards individual substructures, i.e., the temperatures, molecular column densities, and abundances.

\footnotetext{
${ }^{16}$ Lines with $J=196 \rightarrow 195$ and $J=205 \rightarrow 204$ are detected, but neither $J=195 \rightarrow 194$ nor $J=204 \rightarrow 203$ are detected, so these potential detected lines are marked with "?" in Table A.1.
} 
S. Feng et al.: Resolving the chemical substructure of Orion-KL

Table 2. Identification of saturated COMs and complex carbon chains in Orion-KL.

\begin{tabular}{c|cll|l}
\hline \hline Species & Identification & Peak & $\begin{array}{l}\text { Column density } \\
\text { at peak }\left(\mathrm{cm}^{-2}\right)\end{array}$ & Other interferometric detections \\
\hline $\mathrm{CH}_{3} \mathrm{CN}, \mathrm{CH}_{3}^{13} \mathrm{CN}$ & $\sqrt{ }$ & SMA1 & $1.4(17), 9.4(14)$ & $\mathrm{a}, \mathrm{b}, \mathrm{e}, \mathrm{i}$ \\
$\mathrm{CH}_{2} \mathrm{CHCN}$ & $\sqrt{ }$ & North to hotcore & $\geq 5.0(15)$ & $\mathrm{b}, \mathrm{d}, \mathrm{e}$ \\
$\mathrm{CH}_{3} \mathrm{CH}_{2} \mathrm{CN}$ & $\sqrt{ }$ & hotcore & $\geq 1.1(16)$ & $\mathrm{a}, \mathrm{b}, \mathrm{d}, \mathrm{e}, \mathrm{i}, \mathrm{k}$ \\
$\mathrm{CH}_{3} \mathrm{OH}$ & $\sqrt{ }$ & SMA1 \& mm3a & $\geq 2.5(17)$ & $\mathrm{a}, \mathrm{b}, \mathrm{d}, \mathrm{e}, \mathrm{f}, \mathrm{i}, \mathrm{m}$ \\
$\mathrm{HCOOCH}_{3}$ & $\sqrt{ }$ & mm3a \& mm3b & $\geq 9.7(16)$ & $\mathrm{b}, \mathrm{d}, \mathrm{e}, \mathrm{g}, \mathrm{i}, \mathrm{k}$ \\
$\mathrm{CH}_{3} \mathrm{COCH}_{3}$ & $\sqrt{ }$ & SMA1 & $\geq 1.5(16)$ & $\mathrm{c}, \mathrm{i}, \mathrm{k}$ \\
$\mathrm{CH}_{3} \mathrm{OCH}_{3}$ & $\sqrt{ }$ & mm3a \& mm3b & $\geq 2.0(17)$ & $\mathrm{b}, \mathrm{d}, \mathrm{e}, \mathrm{h}, \mathrm{i}, \mathrm{j}$ \\
$\mathrm{CH}_{3} \mathrm{CH}_{2} \mathrm{OH}$ & $\sqrt{ }$ & near SMA1 \& mm3a & $\geq 1.5(16)$ & $\mathrm{d}(\times), \mathrm{e}$ and j (only 1 line) \\
$\left(\mathrm{CH}_{2} \mathrm{OH}\right)_{2}$ & $\times$ & - & - & - \\
$\mathrm{CH}_{2} \mathrm{OHCHO}$ & $\times$ & - & - & g. (some lines $<3 \sigma)$ \\
$\mathrm{HCOOH}$ & $\times$ & - & - & $\mathrm{d}(\times), 1(<3 \sigma), \mathrm{i}($ only from the extended \\
& & & & configuration $)$ \\
$\mathrm{CH}_{3} \mathrm{CHO}$ & $*$ & mm3a \& mm3b & $\geq 1.3(16)$ & $\mathrm{d}(\times), \mathrm{i}(\times), \mathrm{n}(\mathrm{CARMA}$ and ALMA) \\
$\mathrm{C}_{6} \mathrm{H}$ & $*$ & SMA1 & $\geq 1.0(15)$ & - \\
$\mathrm{HC}_{7} \mathrm{~N}$ & $*$ & North to hotcore & $\geq 1.0(16)$ & - \\
\hline
\end{tabular}

Notes. Confirmed COMs are marked with " $\sqrt{ }$ ", tentative detections with “*”, and nondetections with " $\times$ ". At each of their main emission peak (Fig. 5), column density of $\mathrm{CH}_{3} \mathrm{CN}$ is obtained with optical depth correction, while column densities of the rest molecules are obtained at the temperature of an adjacent substructure from synthetic fittings, with an assumption that the species lines are optically thin (we denote them by " $\geq "), x(y)=x \times 10^{y}$.

References. a) Wright et al. (1996); b) Beuther et al. (2005); c) Friedel et al. (2005); d) Friedel \& Snyder (2008); e) Guélin et al. (2008); f) Zapata et al. (2011); g) Favre et al. (2011a); h) Favre et al. (2011b); i) Widicus Weaver \& Friedel (2012); j) Brouillet et al. (2013); k) Peng et al. (2013); 1) Liu et al. (2002); m) Peng et al. (2012a); n) Loomis (priv. comm.).

\subsection{Temperature estimates}

Temperature is commonly used as a benchmark for comparing model results and observations. Since N-, O-, and S-bearing species show different spatial distributions, we estimate their temperatures in each substructure separately.

\subsection{1. $\mathrm{CH}_{3} \mathrm{CN}$}

A good tracer of temperature in high-density gas $\left(n \gtrsim 10^{5} \mathrm{~cm}^{-3}\right)$ is $\mathrm{CH}_{3} \mathrm{CN}$, which is a symmetric-top molecule with no dipole moment perpendicular to the molecular axis, and its $\mathrm{K}$ ladders in rotational levels are excited solely by collisions. Provided that the collision rate is sufficient to thermalize the rotational levels within each $\mathrm{K}$ ladder, the rotational excitation temperature of $\mathrm{CH}_{3} \mathrm{CN}$ is equal to the gas kinetic temperature (Boucher et al. 1980; Wright et al. 1995). Our observation band covers the $J=12 \rightarrow 11$ transitions of $\mathrm{CH}_{3} \mathrm{CN}$ around $220 \mathrm{GHz}$, with upper state energy levels from $\sim 70 \mathrm{~K}$ to $\sim 500 \mathrm{~K}$, yielding a wide range for determining temperatures. If we assume that the emission from all of these transitions originate in the same parcels of gas along the line of sight in LTE, having the same size, velocity, and a single temperature, then rotational temperatures can be derived from the rotational diagrams (Hollis 1982; Loren \& Mundy 1984; Olmi et al. 1993; Goldsmith \& Langer 1999). Traditionally, in the optically thin limit, the level populations are then directly proportional to the line intensities of the $\mathrm{K}$ components. The parameters used to estimate the level populations of $\mathrm{CH}_{3} \mathrm{CN}$ transitions (i.e., the line strength $S_{\mathrm{ul}} \mu^{2}$ (Debye ${ }^{2}$ ) and the lower state energy $\left.E_{\mathrm{L}}\left(\mathrm{cm}^{-1}\right)\right)$ in our data are listed in Table A.2.

The $K=3,6$ lines are from ortho (o-) transitions, which have a statistical weight that is twice as strong as those of the other para (p-) lines. Radiative transitions between o-/p-types are usually prohibited, so we exclude these lines in the optically thin rotation diagram fitting (shown in black lines in Fig. 8, also see Andersson et al. 1984).
Panel 1 of Fig. 8 shows the synthesised fitting under the assumption of optically thin emission in LTE for the HC with the dashed lines. To reduce the number of free parameters, we also assume that all the $K=0-8$ components $^{17}$ have the same line width. A satisfactory fit is obtained to all lines except $K=0,1,2$. Since all transitions were observed simultaneously, calibration errors due to poor pointing or amplitude scaling are not responsible, and the remaining explanations for the deviations are as follows: (1) the $K=0,1$ lines are so close in frequency that they are blended in our fitting by the uniform line width we assumed; (2) it is also likely that lines at $K=2$ are more optically thick.

Compared to $\mathrm{CH}_{3} \mathrm{CN}$, lines of its rare isotopologue $\mathrm{CH}_{3}^{13} \mathrm{CN}$ should be optically thin. However, $\mathrm{CH}_{3}^{13} \mathrm{CN}\left(12_{2} \rightarrow 11_{2}\right)$ is the only transition that is not blended with the broad line wings of $\mathrm{CH}_{3} \mathrm{CN}$ in substructures other than $\mathrm{HC}$. Even in the $\mathrm{HC}$, only the $K=2,6$, and 7 lines are unblended (Panel 7 of Fig. 8). Excluding the o-type lines $(K=3,6)$, the rotation temperatures in $\mathrm{HC}$ we derive from the main and rare isotopologues of $\mathrm{CH}_{3} \mathrm{CN}$ have large differences (black fittings in Panels 2 and 8 of Fig. 8). Optical depth seems to be the most likely reason for such inconsistency, so to investigate this hypothesis, we use the following iterative approach to correct the optical depth of $\mathrm{CH}_{3} \mathrm{CN}$ lines and derive the opacity-corrected rotation temperature $T_{\text {rot, } p}(p=$ $\mathrm{HC}, \mathrm{mm} 2, \mathrm{~mm} 3 \mathrm{a}, \mathrm{mm} 3 \mathrm{~b}, \mathrm{NE}$ ) of each substructure (red fittings in Panels 2-6 of Fig. 8):

(1) Obtain the rotation temperature $T_{1, p}$ from weighted least squares fit to Boltzmann diagram, assuming that all the p-type $\mathrm{CH}_{3} \mathrm{CN}$ lines are optically thin (see fit to the black dots in Panels 2-6 of Fig. 8);

(2) Estimate the optical depth of $\mathrm{CH}_{3} \mathrm{CN}\left(12_{2} \rightarrow 11_{2}\right) \tau_{K, 1}(K=2)$ by comparing its main beam temperature with that of $\mathrm{CH}_{3}^{13} \mathrm{CN}\left(12_{2} \rightarrow 11_{2}\right)$, and calculate the column density of line $12_{2} \rightarrow 11_{2}$ at $T_{1, p}$ with the optical depth correction.

${ }^{17}$ The $K=9$ line is blended by strong ${ }^{13} \mathrm{CO}(2 \rightarrow 1)$ emission. 

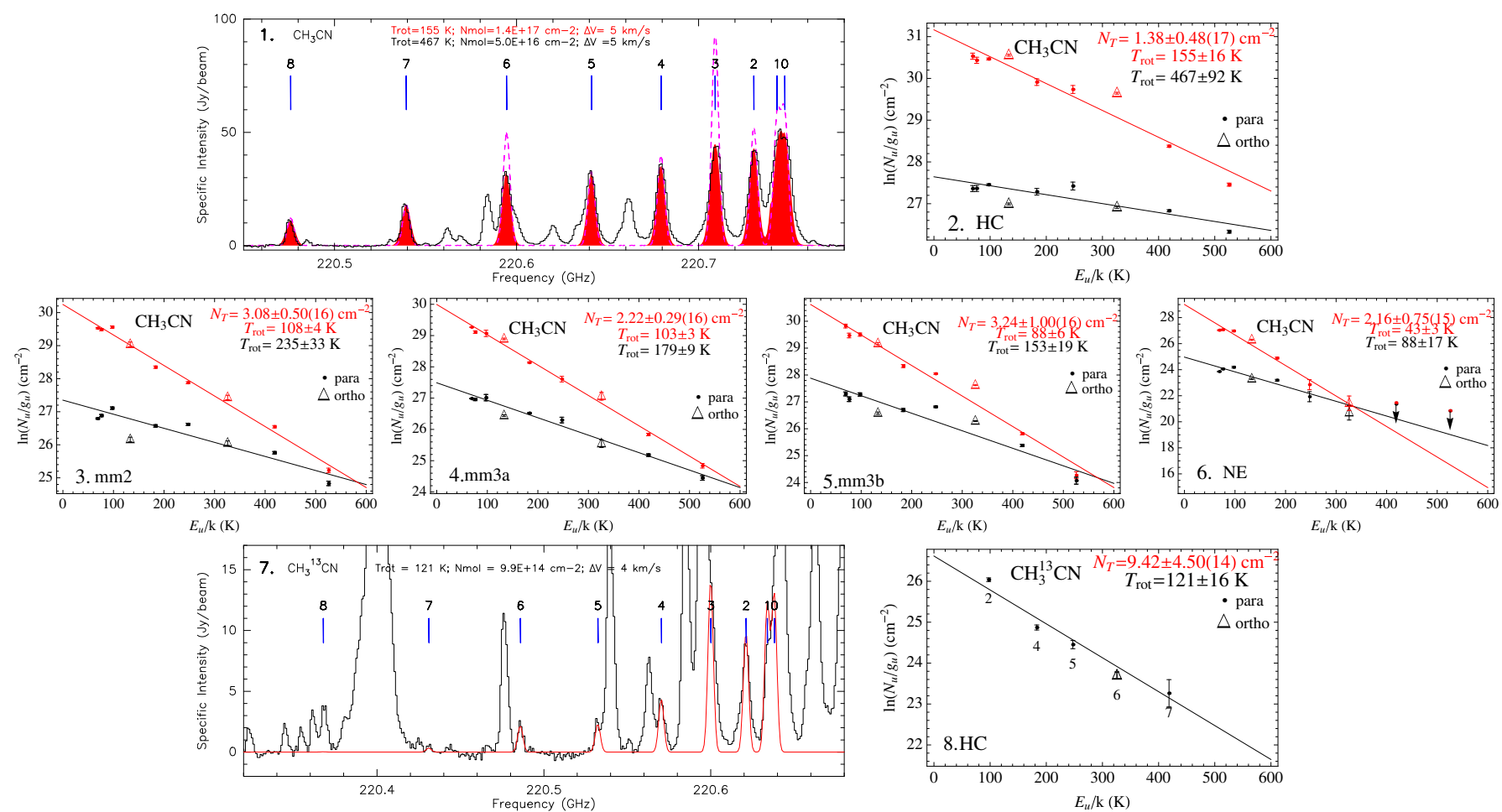

Fig. 8. Panel 1): $\mathrm{CH}_{3} \mathrm{CN}$ spectrum towards the $\mathrm{HC}$ in black, LTE model fit in dashed purple, and fitting with optical depth correction in filled red. Panels 2)-6): rotation diagram of $\mathrm{CH}_{3} \mathrm{CN}$ derived for each substructure, with dots for $\mathrm{p}-\mathrm{CH}_{3} \mathrm{CN}$ and triangles for o- $\mathrm{CH}_{3} \mathrm{CN}$. Black fittings are only for the p-lines when assuming they are optically thin, and red fittings are for both o- and p-lines by optical depth correction. Panel 7): $\mathrm{CH}_{3}^{13} \mathrm{CN}$ spectrum towards the $\mathrm{HC}$ in black and the LTE model fit in red. Panel 8): rotation diagram of $\mathrm{CH}_{3}^{13} \mathrm{CN}$ in $\mathrm{HC}$ with black dots (p-) and triangles (o-) from observation, and fitting is o-lines excluded by assuming lines are optically thin. For lines without detection, an upper limit equal to the $3 \sigma \mathrm{rms}$ is shown as arrow. The estimated temperature and total column density from each fitting is indicated in each panel. Errors are derived from scatter in the data points.

Then, estimate the total column density of $\mathrm{CH}_{3} \mathrm{CN}$ as $\mathrm{N}_{1, p}$ assuming LTE (see Sect. 4.2 for more details about the correction);

(3) With $T_{1, p}$ and $N_{1, p}$ as trial input parameters, estimate the optical depth for the remaining transitions in the $\mathrm{CH}_{3} \mathrm{CN}$ ladder $\tau_{K, 1(K=0-8)}$ with the RADEX code package ${ }^{18}$ (van der Tak et al. 2007), then correct their column densities line by line (dots and triangles in red in Fig. 8) and obtain a new rotation temperature $T_{2, p}$;

(4) Repeat step (2)-(3) until $\left(\left|T_{\mathrm{n}, p}-T_{n-1, p}\right|\right) / T_{n-1, p} \leq 10 \%(n=$ 2, 3,..; see fittings in red in Fig. 8).

The above approach ${ }^{19}$ can be applied to sources HC, mm2 mm3a, mm3b, and $\mathrm{NE}$, where the $\mathrm{CH}_{3} \mathrm{CN}$ ladders and $\mathrm{CH}_{3}^{13} \mathrm{CN}\left(12_{2} \rightarrow 11_{2}\right)$ can be clearly detected ${ }^{20}$. In SR and OF1N(S), the $\mathrm{CH}_{3} \mathrm{CN}$ lines are below the $3 \sigma$ detection threshold, so we assume the rotation temperature in these positions to be the same as that in NE.

\footnotetext{
18 RADEX is a one-dimensional non-LTE radiative transfer code, providing an alternative to the widely used rotation diagram method. Without requiring the observation of many optically thin emission lines, it can be used to roughly constrain the excitation temperature in addition to the column density.

19 In reality, molecules do not distribute homogeneously in the clouds, and they are far from being homogeneous in density and temperature. The ultimate best-fit requires a sophisticated, fully 3D physico-chemoLRT modelling, which is beyond the scope of our paper. Our iterative approach is between realism and feasibility of the modelling, which fits both o-/p- $\mathrm{CH}_{3} \mathrm{CN}$ well.

20 The detection of $\mathrm{CH}_{3}^{13} \mathrm{CN}\left(12_{2} \rightarrow 11_{2}\right)$ in NE is $4 \sigma$.
}

\subsection{2. $\mathrm{HCOOCH}_{3}, \mathrm{CH}_{3} \mathrm{OH}$, and ${ }^{34} \mathrm{SO}_{2}$}

$\mathrm{HCOOCH}_{3}$ has the largest number of lines detected in our dataset. From satisfactory synthetic fitting (especially for lines in the torsionally excited state $v=1$, Fig. A.2), we can reasonably assume that most of its lines are optically thin. Line emissions from this species are not contaminated by $\mathrm{N}$-bearing species owing to their different spatial distributions, so we use them to derive a second set of temperatures.

On the other hand, $\mathrm{CH}_{3} \mathrm{OH}$ and $\mathrm{H}_{2}^{13} \mathrm{CO}$ have an unusual spatial distribution (dual peaks /extended emissions at both $\mathrm{HC}$ and $\mathrm{mm} 3 \mathrm{a}(\mathrm{b})$, and tail towards the SE), which may be affected by their different temperatures. Since multiple transitions of $\mathrm{CH}_{3} \mathrm{OH}$ are available in our data, we can use them to derive a third temperature set.

For S-bearing species, their extended emission indicates they may be sensitive to the ambient shock environment, so their temperature in each substructure may be different from the other species. However, in our data, some of the S-bearing species have only a single transition $\left(\mathrm{O}^{13} \mathrm{CS},{ }^{13} \mathrm{CS}\right)$, and other lines may not be optically thin ( $\mathrm{SO}_{2}, \mathrm{SO}$, and OCS). Because ${ }^{34} \mathrm{SO}_{2}$ is the only S-bearing rare isotopologue that has multiple transitions in our observations, we applied temperatures derived from transitions of ${ }^{34} \mathrm{SO}_{2}$ to the other S-bearing species.

Using the lines list in Table A.3, we derived the rotation temperatures in each substructure from $\mathrm{HCOOCH}_{3}, \mathrm{CH}_{3} \mathrm{OH}$, and ${ }^{34} \mathrm{SO}_{2}$ (Figs. 9I-III) and list these temperature sets in bold face in Table 3. Temperatures derived from $\mathrm{HCOOCH}_{3}$ lines in the ground and torsionally excited states separately have some differences, but they do not deviate significantly from temperatures 
derived from all of its lines. Therefore, we use the "mixed" temperatures in the end.

\subsubsection{The other organics}

In addition, since different organic molecules have specific spatial distributions, we also derive the rotation temperatures for the species that have multiple transitions with significantly different $E_{\mathrm{u}} / k_{\mathrm{B}}$ detected in our dataset (Figs. 9IV-VI, listed in Table A.3). In the condensations $\mathrm{HC}, \mathrm{mm} 2, \mathrm{~mm} 3 \mathrm{a}$, and $\mathrm{mm} 3 \mathrm{~b}$, rotation temperatures of $\mathrm{HNCO}$ are similar to those derived from $\mathrm{CH}_{3} \mathrm{CN}$ within uncertainties; and rotation temperatures derived from $\mathrm{CH}_{3} \mathrm{OCH}_{3}$ and $\mathrm{CH}_{3} \mathrm{CH}_{2} \mathrm{OH}$ are close to $\mathrm{HCOOCH}_{3}(v=1)$ within uncertainties. Therefore, temperature sets from $\mathrm{CH}_{3} \mathrm{CN}$ and $\mathrm{HCOOCH}_{3}$ are applicable to the rest $\mathrm{N}$ - and O-bearing species (including simple molecules and COMs) in estimating their column densities.

Although temperatures derived from different species at the same substructure vary, $\mathrm{HC}$ is consistently the warmest substructure, while $\mathrm{mm} 2, \mathrm{~mm} 3 \mathrm{a}$, and $\mathrm{mm} 3 \mathrm{~b}$ are slightly cooler. In the outflow regions, there are no sufficiently strong lines for species other than $\mathrm{CH}_{3} \mathrm{CN}$ to derive the temperature, which may suggest these regions are cooler, exhibiting similar chemical properties.

\subsection{Column densities}

\subsubsection{Molecular column densities}

For all the species we detected, we calculate their column densities (from both main and rare isotopologues) from the integrated spectral line intensities as below.

The optical depth $\tau_{v}$ (in velocity) along the line of sight is (Zeng et al. 2006, Eq. (A.25)):

$\tau_{v}=\frac{c^{3}}{8 \pi v^{3}} A_{\mathrm{ul}} N_{\mathrm{u}}\left(\mathrm{e}^{\frac{h v}{k_{\mathrm{B}} T_{\mathrm{rot}}}}-1\right) \Phi(v)$,

where $c$ is the speed of light, $k_{\mathrm{B}}$ the Boltzmann constant, $h$ the Planck constant, $v$ the line rest frequency, $\Phi(v)$ the distribution function of the line shape in terms of velocity $v$. Here, $A_{\mathrm{ul}}$ is the average spontaneous emission rate from the upper state $E_{\mathrm{u}}$ into the lower state $E_{1}$, and it is calculated from line strength $S_{\mathrm{ul}}$ and dipole moment $\mu$ as

$A_{\mathrm{ul}}=\frac{64 \pi^{4} v^{3}}{3 h c^{3}} \frac{S_{\mathrm{ul}} \mu^{2}}{g_{\mathrm{u}}}$.

After integrating the optical depth over the observed linewidth, $\int \Phi(v) \mathrm{d} v=1$, and the column density $N_{\mathrm{u}}$ of the line at $v \mathrm{~Hz}$ is

$N_{\mathrm{u}}=\frac{8 \pi v^{3}}{c^{3} A_{\mathrm{ul}}} \frac{1}{\exp \left(\frac{h v}{k_{\mathrm{B}} T_{\text {rot }}}\right)-1} \int \tau_{v} \mathrm{~d} v . \quad\left(\mathrm{cm}^{-2}\right)$.

When assuming LTE, the total column density of all transitions for a molecule $\alpha$ is

$N_{T, \alpha}=\frac{N_{\mathrm{u}}}{g_{\mathrm{u}}} Q\left(T_{\mathrm{rot}}\right) \mathrm{e}^{\frac{E_{\mathrm{u}}}{k_{\mathrm{B}} T_{\mathrm{rot}}}} \quad\left(\mathrm{cm}^{-2}\right)$

where $Q\left(T_{\text {rot }}\right)$ is the partition function for the given (rotational) excitation temperature of each sources, which is interpolated from a table of partition functions for fixed temperatures, obtained from CDMS/JPL.
Here, $S_{\mathrm{ul}} \mu^{2}$ can be calculated from CDMS/JPL line intensity $\ell\left(T_{\text {rot }}\right)$ (Pickett et al. 1998),

$$
\begin{aligned}
S_{\mathrm{ul}} \mu^{2}= & \frac{3 h c}{8 \pi^{3} v} \ell\left(T_{\text {rot }}\right) Q\left(T_{\text {rot }}\right)\left(\mathrm{e}^{-\frac{E_{1}}{k_{\mathrm{B}} T_{\mathrm{rot}}}}-\mathrm{e}^{-\frac{E_{\mathrm{u}}}{k_{\mathrm{B}} T_{\mathrm{rot}}}}\right)^{-1} \\
= & 2.4025 \times 10^{10}\left[\frac{v}{\mathrm{~Hz}}\right]^{-1}\left[\frac{\ell(300 \mathrm{~K})}{\mathrm{nm}^{2} \mathrm{MHz}}\right] Q(300 \mathrm{~K}) \\
& \times\left[\mathrm{e}^{-\frac{E_{1}}{k_{\mathrm{B}} 300 \mathrm{~K}}}-\mathrm{e}^{-\frac{E_{\mathrm{u}}}{k_{\mathrm{B}} 300 \mathrm{~K}}}\right]^{-1} \text { Debye }^{2}
\end{aligned}
$$

For the emission lines, we assume the Rayleigh-Jeans approximation $\left(\frac{h v}{k_{\mathrm{B}} T_{\mathrm{rot}}} \ll 1\right)$ is valid, therefore Eqs. (3) and (4) can be simplified as (Zeng et al. 2006, Eq. (4.26); also see Wilson et al. 2009),

$\frac{N_{\mathrm{u}}}{g_{\mathrm{u}}} \cong \frac{3 k_{\mathrm{B}}}{8 \pi^{3} v S_{\mathrm{ul}} \mu^{2}} T_{\text {rot }} \int \tau_{v} \mathrm{~d} v \quad\left(\mathrm{~cm}^{-2}\right)$
$N_{\mathrm{T}, \alpha}=\frac{k_{\mathrm{B}}}{h c} \frac{\left(\mathrm{e}^{\frac{h \nu}{k_{\mathrm{B}} T_{\mathrm{rot}}}}-1\right)}{\ell\left(T_{\text {rot }}\right)} T_{\text {rot }} \int \tau_{v} \mathrm{~d} v \quad\left(\mathrm{~cm}^{-2}\right)$.

Assuming that the observed emission in each substructure is homogeneous and fills the combined beam, the integration of measured main beam brightness temperature within the velocity range $\int T_{\mathrm{B}}(v) \mathrm{d} v$ can be substituted for the last term in the above equation:

$T_{\text {rot }} \int \tau_{v} \mathrm{~d} v \cong \frac{\tau_{\alpha, 0}}{1-\mathrm{e}^{-\tau_{\alpha, 0}}} \int T_{\mathrm{B}}(v) \mathrm{d} v \quad\left(\mathrm{~K} \mathrm{~cm} \mathrm{~s}^{-1}\right)$

where $\tau_{\alpha, 0}$ is the optical depth at line centre, and $\int T_{\mathrm{B}}(v) \mathrm{d} v$ is measured from Gaussian/hyperfine structure (HFS) fitting by using Gildas software package.

The column densities and related uncertainties of species listed in Table $3\left(\mathrm{CH}_{3} \mathrm{CN}\left(\mathrm{CH}_{3}^{13} \mathrm{CN}\right), \mathrm{HNCO}, \mathrm{HCOOCH}_{3}\right.$, $\mathrm{CH}_{3} \mathrm{OH}, \mathrm{CH}_{3} \mathrm{OCH}_{3}, \mathrm{CH}_{3} \mathrm{CH}_{2} \mathrm{OH}$, and ${ }^{34} \mathrm{SO}_{2}$ ) can be estimated directly from their rotation diagrams and the scatterings of the data points (Fig. 9). For the species whose transitions are not sufficient to derive the rotation diagrams, we estimate their column densities by assuming that transitions of related species have the same $T_{\text {rot }}$ :

- N-bearing species have similar temperatures to those derived from the opacity-corrected $\mathrm{CH}_{3} \mathrm{CN}$,

- S-bearing species have similar temperatures to ${ }^{34} \mathrm{SO}_{2}$,

- O-bearing species have similar temperatures to $\mathrm{HCOOCH}_{3}$,

- given the similarly unusual spatial distribution between $\mathrm{H}_{2}^{13} \mathrm{CO}$ and $\mathrm{CH}_{3} \mathrm{OH}$, we also estimate the column densities of $\mathrm{H}_{2}^{13} \mathrm{CO}$ at temperatures derived from $\mathrm{CH}_{3} \mathrm{OH}$.

In general, we assume that the observed transitions are optically thin $\left(\tau_{\alpha, 0} \leq 1, \frac{\tau_{\alpha, 0}}{1-\mathrm{e}^{-\tau_{\alpha, 0}}} \approx 1\right)$. However, for several molecules with large abundances, we observe the corresponding transitions of their main and rare isotopologues, e.g., OCS $(J=19 \rightarrow 18)-$ $\mathrm{O}^{13} \mathrm{CS}(19 \rightarrow 18),{ }^{13} \mathrm{CO}(2 \rightarrow 1)-\mathrm{CO}(2 \rightarrow 1)-\mathrm{C}^{18} \mathrm{O}(2 \rightarrow 1)$ and $\mathrm{CH}_{3}^{13} \mathrm{CN}\left(12_{2} \rightarrow 11_{2}\right)-\mathrm{CH}_{3} \mathrm{CN}\left(12_{2} \rightarrow 11_{2}\right)$. By measuring the ratio between main beam brightness temperature of the main line $T_{\mathrm{B}, \alpha, 0}$ and its rare isotopologue $T_{\mathrm{B}, \beta, 0}$, we can estimate the optical depth at line centre of a given transition $\tau_{\alpha, 0}$ (Myers et al. 1983):

$\frac{1-\exp \left(-\tau_{\alpha, 0} / \mathfrak{R}^{\alpha}\right)}{1-\exp \left(-\tau_{\alpha, 0}\right)} \approx \frac{T_{\mathrm{B}, \beta, 0}}{T_{\mathrm{B}, \alpha, 0}}$ 


\section{I. $\mathrm{HCOOCH}_{3}$}
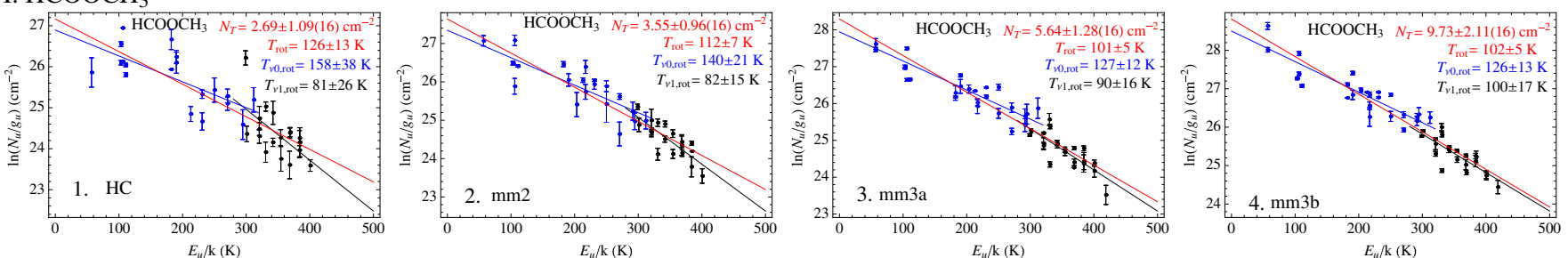

\section{II. $\mathrm{CH}_{3} \mathrm{OH}$}
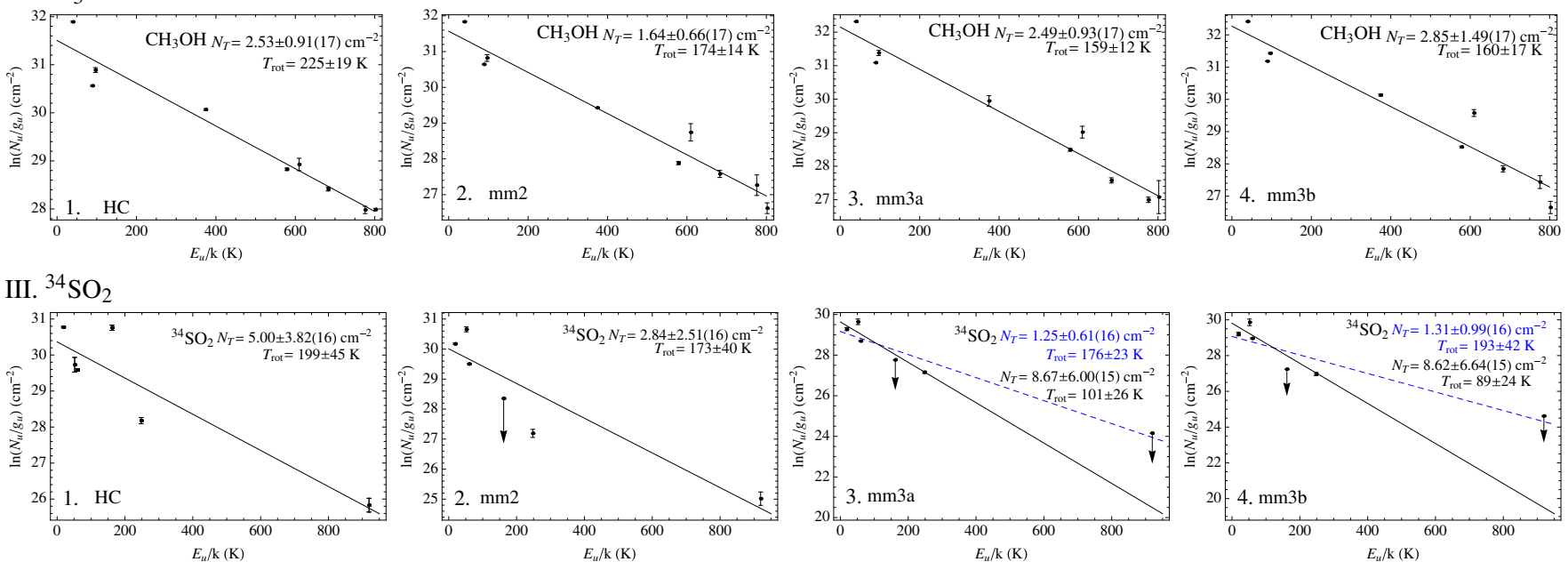

\section{HNCO}
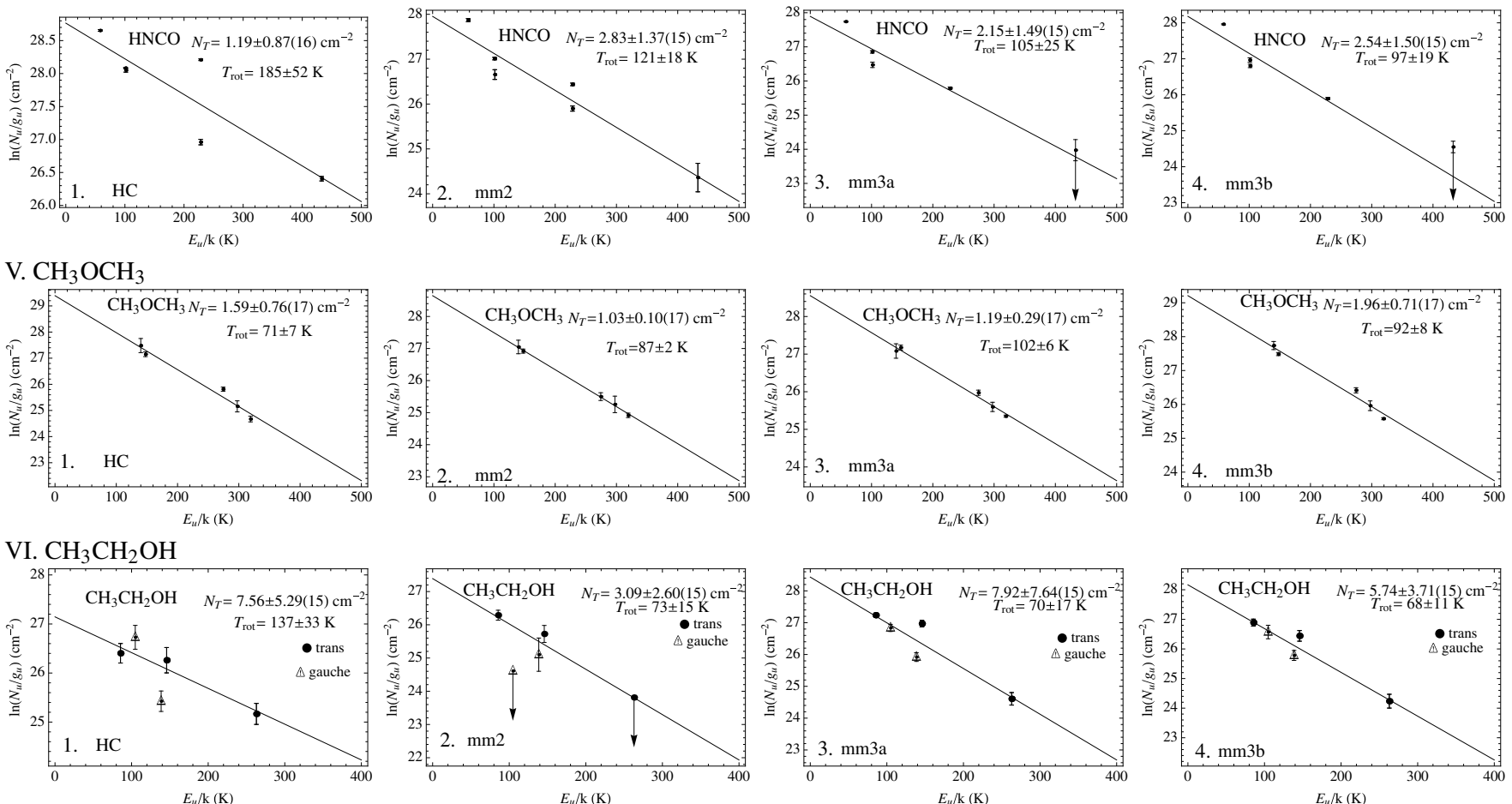

Fig. 9. Rotation diagrams of the N-, O-, and S-bearing species with multiple unblended transitions detection in $\mathrm{HC}$, $\mathrm{mm} 2$, mm3a, and $\mathrm{mm} 3 \mathrm{~b}$. Panels in row I show two states of $\mathrm{HCOOCH}_{3}$ lines, with the blue and black dots from observation of ground state $(v=0)$ and torsionally excited state $(v=1)$ lines, respectively. With the optically thin assumption, fittings for each state are plotted in blue or black, respectively, and fittings for all the transitions are plotted in red. Non-detection of $\mathrm{HCOOCH}_{3}$ lines are not shown. Panels in rows II-VI show fittings with the optically thin assumption, with $3 \sigma$ upper limits from non-detections plotted as arrows. Blue dashed lines in row III are fittings with upper limits included. The estimated temperature and total column density from each fitting is indicated in each panel. Errors are derived from scatters in the data points.

where $\mathfrak{R}_{\alpha}$ is the intrinsic abundance of the main isotope (e.g., ${ }^{12} \mathrm{C}$ ) compared to its rare isotope (e.g., ${ }^{13} \mathrm{C}$ ) in the ISM (Table 4, e.g., Wilson \& Rood 1994; Chin et al. 1996).
Using the above method, we calculate the column densities of 25 isotopologues (for both optically thin and thick cases) in each substructure, and present them in Tables A.5I-A.7I. 
S. Feng et al.: Resolving the chemical substructure of Orion-KL

Table 3. Rotation temperature sets for each substructure derived from species, each of which has $>4$ transitions with significantly different $E_{\mathrm{u}} / k_{\mathrm{B}}$ detected in our data.

\begin{tabular}{c|lllll}
\hline \hline Species & $\mathrm{HC}(\mathrm{K})$ & $\mathrm{mm} 2(\mathrm{~K})$ & $\mathrm{mm} 3 \mathrm{a}(\mathrm{K})$ & $\mathrm{mm} 3 \mathrm{~b}(\mathrm{~K})$ & $\mathrm{NE}(\mathrm{K})$ \\
\hline $\mathbf{C H}_{\mathbf{3}} \mathbf{C N}$ & $\mathbf{1 5 5} \pm \mathbf{1 6}$ & $\mathbf{1 0 8} \pm \mathbf{4}$ & $\mathbf{1 0 3} \pm \mathbf{3}$ & $\mathbf{8 8} \pm \mathbf{6}$ & $\mathbf{4 3} \pm \mathbf{3}$ \\
$\mathrm{CH}_{3}^{13} \mathrm{CN}$ & $\mathbf{1 2 1} \pm \mathbf{1 6}$ & - & - & - & - \\
$\mathrm{HNCO}$ & $185 \pm 52$ & $121 \pm 18$ & $105 \pm 25$ & $97 \pm 19$ & - \\
\hline $\mathbf{H C O O C H}_{\mathbf{3}}$ & $\mathbf{1 2 6} \pm \mathbf{1 3}$ & $\mathbf{1 1 2} \pm \mathbf{7}$ & $\mathbf{1 0 1} \pm \mathbf{5}$ & $\mathbf{1 0 2} \pm \mathbf{5}$ & - \\
$\mathbf{C H}_{\mathbf{3}} \mathbf{O H}$ & $\mathbf{2 2 5} \pm \mathbf{1 9}$ & $\mathbf{1 7 4} \pm \mathbf{1 4}$ & $\mathbf{1 5 9} \pm \mathbf{1 2}$ & $\mathbf{1 6 0} \pm \mathbf{1 7}$ & - \\
$\mathrm{CH}_{3} \mathrm{OCH}_{3}$ & $71 \pm 7$ & $87 \pm 2$ & $102 \pm 6$ & $92 \pm 8$ & - \\
$\mathrm{CH}_{3} \mathrm{CH}_{2} \mathrm{OH}$ & $137 \pm 33$ & $73 \pm 15$ & $70 \pm 17$ & $68 \pm 11$ & - \\
\hline${ }^{\mathbf{3 4}} \mathbf{S O}_{\mathbf{2}}$ & $\mathbf{1 9 9} \pm \mathbf{4 5}$ & $\mathbf{1 7 3} \pm \mathbf{4 0}$ & $\mathbf{1 0 1} \pm \mathbf{2 6}$ & $\mathbf{8 9} \pm \mathbf{2 4}$ & - \\
\hline
\end{tabular}

Notes. The substructures where there are not enough sufficient lines to derive the rotation temperatures are denoted by "-". Temperatures in bold face are used to estimate the column densities and abundances of species whose rotation diagrams are unable to be derived.

In Table 4, we list the transitions for which we corrected their optical depths. We found that CO $(2 \rightarrow 1)$, OCS $(19 \rightarrow 18)$, and $\mathrm{CH}_{3} \mathrm{CN}\left(12_{2} \rightarrow 11_{2}\right)$ are significantly optically thick $(\tau=$ $6-117)$ in all substructures where they are detected. The validity of the assumption that the remaining molecular lines are optically thin is discussed in Sect. 4.4.

In addition, the main emission peak(s) of some COMs $\left(\mathrm{CH}_{3} \mathrm{COCH}_{3}, \mathrm{CH}_{3} \mathrm{CH}_{2} \mathrm{OH}, \mathrm{CH}_{2} \mathrm{CHCN}, \mathrm{C}_{6} \mathrm{H}\right.$, and $\left.\mathrm{HC}_{7} \mathrm{~N}\right)$ do not strictly coincide with either of the continuum peaks. In determining their column densities at their main emission peaks, we use their strongest transitions (shown in Fig. 5) and the temperature from an adjacent substructure. Lines are assumed to be optically thin, and molecular column densities could be underestimated if this assumption is incorrect, so the lower limits are given in Table 2.

\subsection{2. $\mathrm{H}_{2}$ column densities}

In Orion-KL, at $1.3 \mathrm{~mm}$ wavelength, the continuum emission is dominated by dust-neutral gas emission (Beuther et al. 2004). Assuming the dust emission at mm wavelengths is optically thin and coupled with the gas, which has a single excitation temperature, $T_{\text {ex }} \sim T_{\text {rot }}=T_{\text {dust }}$ (Hildebrand 1983), we use the SMA-only data to calculate the $\mathrm{H}_{2}$ column densities for different substructures, according to Schuller et al. (2009) :

$N_{\mathrm{H}_{2}}=\frac{I_{v} R}{B_{v}\left(T_{\text {dust }}\right) \Omega_{a} \kappa_{v} \mu m_{\mathrm{H}}} \quad\left(\mathrm{cm}^{-2}\right)$

where $I_{v}$ is the peak specific intensity for each substructure on the continuum (in Jy beam ${ }^{-1}$, taken to be the $3 \sigma$ upper limit in the outflow regions); $R$ is the isothermal gas to dust mass ratio (taken to be 150 , from Draine 2011); $B_{v}\left(T_{\text {dust }}\right)$ is the Planck function for a dust temperature $T_{\text {dust }} ; \Omega_{\mathrm{a}}$ is the solid angle of the SMA beam (in $\operatorname{rad}^{2}$ ); $\kappa_{v}=1.0 \mathrm{~cm}^{2} \mathrm{~g}^{-1}$ is the dust absorption coefficient at $1.3 \mathrm{~mm}$ (assuming a model of agglomerated grains with thin ice mantles ${ }^{21}$ for densities $10^{7} \mathrm{~cm}^{-3}$ (Blake et al. 1987), extrapolated from Ossenkopf \& Henning (1994); $\mu$ is the mean

\footnotetext{
21 At around 100-150 K, water ice starts to be evaporated. However, in the line of sight, the envelope of the protostar may not have the same temperature at different radii, so ice may not be completely evaporated. Therefore, without knowing the real geometry of the source, we assume the dust absorption coefficient according to the "thin ice mantle" case. Moreover, this assumption is consistent with the modelling result in Gerner et al. (2014). In the extremely case, if we assume the ice is completely evaporated, the $\mathrm{H}_{2}$ column density derived from continuum will be $2-4$ times lower than our current estimation.
}

molecular weight of the ISM, which is assumed to be 2.33; and $m_{\mathrm{H}}$ is the mass of an hydrogen atom $\left(1.67 \times 10^{-24} \mathrm{~g}\right)$. The results are reported in column $N_{\mathrm{H}_{2}, 1}$ of Table 1 .

From the SMA-only observations, the total continuum flux at $1.3 \mathrm{~mm}$ is $22.6 \mathrm{Jy}$. However, extrapolating the flux $S_{v}$ from existing SCUBA data (Di Francesco et al. 2008) at $450 \mu \mathrm{m}$ (28 $700 \mathrm{Jy})$ and $850 \mu \mathrm{m}(2090 \mathrm{Jy})$, the total flux should be closer to $\sim 400 \mathrm{Jy}$ at $1.3 \mathrm{~mm}$ (assuming $S_{v} \propto v^{4}$, Johnstone \& Bally 1999). The difference implies that $>90 \%$ of the flux has been filtered out by the SMA observations. In contrast, $\mathrm{C}^{18} \mathrm{O}(2 \rightarrow 1)$ from a combination of $30 \mathrm{~m}$ and SMA observations is detected towards all the substructures, and it should not suffer from the same spatial filtering as the SMA-only continuum data. As a result, it is a more reliable probe of the total column density of gas in the cloud at all spatial scales. Moreover, as a rare isotopologue line, it can be reasonably assumed to be optically thin, so we use it to make a second independent estimation of the $\mathrm{H}_{2}$ column density in all the substructures. By assuming a constant abundance in all substructures $\frac{N_{\mathrm{C}^{18} \mathrm{O}}}{N_{\mathrm{H}_{2}}}=\frac{N_{\mathrm{C}^{18} \mathrm{O}}}{N_{\mathrm{CO}}} \frac{N_{\mathrm{CO}}}{N_{\mathrm{H}_{2}}} \sim \frac{10^{-4}}{560} \sim 1.79 \times 10^{-7}$ (Wilson \& Rood 1994), $\mathrm{H}_{2}$ column densities can be calculated from the column densities of optically thin $\mathrm{C}^{18} \mathrm{O}$ emission (reported in the column $N_{\mathrm{H}_{2}, 2}$ of Table 1).

\subsection{Molecular abundances}

Subsequently, to study the chemical properties on the small scale of Orion-KL, we derived the molecular abundances with respect to $\mathrm{H}_{2}$ (converted from $\mathrm{C}^{18} \mathrm{O}$ ) in each substructure (Tables A.5II-A.7II).

Figure 10 presents the molecular column densitiy and the abundance of each species with respect to $N_{\mathrm{H}_{2}, 2}$. Generally, all the molecules have higher column densities in the central condensations ( $\mathrm{HC}, \mathrm{mm} 2, \mathrm{~mm} 3 \mathrm{a}$, and $\mathrm{mm} 3 \mathrm{~b}$ ) than the extended regions in the outflows (NE, SR, OF1N, and OF1S). While the abundances of some species have relatively small variations among the substructures (e.g., $\mathrm{SO}, \mathrm{CO}, \mathrm{C}^{18} \mathrm{O}$, and ${ }^{13} \mathrm{CO}$ ), some vary substantially. Specifically, N-bearing species are significantly underabundant in the SR and the outflow regions; O-bearing organic molecules containing $\leq 6$ atoms (e.g., $\mathrm{H}_{2}^{13} \mathrm{CO}, \mathrm{CH}_{2} \mathrm{CO}, \mathrm{CH}_{3} \mathrm{OH}$ ) have more extended emission across all the substructures, with nearly constant large abundances in all the substructures except for NE. The S-bearing molecules are an intermediate case: most of them are not detected in the high-velocity outflow, but are clearly detected in $\mathrm{NE}\left(\mathrm{OCS}, \mathrm{SO}_{2}\right.$, 
${ }^{34} \mathrm{SO}_{2},{ }^{13} \mathrm{CS}$ ) and (or) the SR (SO), with lower abundance. Such abundance differentiation among species is consistent with the spatial differentiation shown in Fig. 4.

\subsection{Error budget}

In this section, we consider the effect that the various assumptions that we made in our quantitative analysis have on the results presented in this paper.

1. $T_{\text {rot }}$ (rotation temperature): the traditional rotation diagram method is subject to a number of uncertainties owing to various assumptions, e.g, line emission is assumed to be optically thin and LTE is assumed to apply (see discussion in Snyder et al. 2005; Herbst \& van Dishoeck 2009). If these assumptions are valid, the points on the population diagram will be well-fit by a straight line, with the slope proportional to the negative reciprocal of the temperature. However, this is not the case (see Fig. 8). According to Table 2, the volumn density in each substructure is $\sim 10^{7} \mathrm{~cm}^{-3}$ (assuming a source size $\sim 10$ times of the beam size, see also Blake et al. 1987; Plume et al. 2012), so LTE can be a reasonable assumption (except for such extreme cases as masing and sub/super thermal excited conditions), and the main reason for the fittings with uncertainties is the optical depth of the used lines.

(1) In Sect. 4.1.1, we obtain the optical depth of $\mathrm{CH}_{3} \mathrm{CN}\left(12_{2} \rightarrow 11_{2}\right)$ by comparing its observed main beam brightness temperature to that of $\mathrm{CH}_{3}^{13} \mathrm{CN}\left(12_{2} \rightarrow 11_{2}\right)$. Then, we use this optical depth as a standard in non-LTE radiative transfer fitting and derive a lower $\mathrm{T}_{\text {rot }}$ in each substructure. In the $\mathrm{HC}$, the rotational temperatures derived from $\mathrm{CH}_{3} \mathrm{CN}(155 \pm 16 \mathrm{~K})$ and $\mathrm{CH}_{3}^{13} \mathrm{CN}(121 \pm 16 \mathrm{~K})$ are slightly different, but they agree within the uncertainties, and column densities of N-bearing species are insensitive to temperature uncertainties of this magnitude (Table A.6I). On the other hand, temperatures derived from HNCO agree with the above $T_{\text {rot }}$ within the uncertainties, so our approach should be applicable.

(2) In Sect. 4.1.2, $T_{\text {rot }}$ for most O-bearing species are assumed to be similar to those derived from $\mathrm{HCOOCH}_{3}$. In general, the temperatures derived from two different states of $\mathrm{HCOOCH}_{3}$ lines are consistent, while the torsionally excited $\mathrm{HCOOCH}_{3}(v=1)$ lines appear to trace lower temperatures than lines in the ground state $(v=0)$, because LTE assumption may not be applied in sub-thermal conditions (see also Demyk et al. 2008). In addition, we derived a third temperature set from $\mathrm{CH}_{3} \mathrm{OH}$, which is in general higher than those derived from $\mathrm{HCOOCH}_{3}, \mathrm{CH}_{3} \mathrm{OCH}_{3}$, and $\mathrm{CH}_{3} \mathrm{CH}_{2} \mathrm{OH}$. Synthetic spectrum fitting (Fig. A.2) indicates that some lines of $\mathrm{CH}_{3} \mathrm{OH}$ may be optically thick, which may explain the higher measured $T_{\text {rot }}$. On the other hand, $\mathrm{CH}_{3} \mathrm{OH}$ lines have broader line widths $\left(\sim 5-7 \mathrm{~km} \mathrm{~s}^{-1}\right)$ than $\mathrm{HCOOCH}_{3}$ lines $\left(\sim 3-5 \mathrm{~km} \mathrm{~s}^{-1}\right)$ in the central condensations, implying that they trace gas with different temperatures.

(3) For S-bearing species, $T_{\text {rot }}$ derived from ${ }^{34} \mathrm{SO}_{2}$ varies from $\mathrm{HC}$ through $\mathrm{mm} 2$ to $\mathrm{mm} 3 \mathrm{a}$ and $\mathrm{mm} 3 \mathrm{~b}$. However, detected lines in $\mathrm{mm} 3 \mathrm{a}$ and $\mathrm{mm} 3 \mathrm{~b}$ are not sufficient to derive a reliable temperature, because we lose the constraint of fittings from the non-detection line with high $E_{\mathrm{u}} / k_{\mathrm{B}}$ at $229.987 \mathrm{GHz}$ (Fig. 9III). Nevertheless, we estimate the temperature upper limits for both substructures by including the non-detected lines (dashed blue fittings), and find the differences for column densities derived at temperatures from 89-101 K (non-detection are excluded in fittings) to 176-193 K (upper limits, columns in italic font in Table A.7I) are by a factor of $<3$. The insensitivity of S-bearing column densities to this temperature regime may also explain the slightly higher $T_{\text {rot }}$ with large uncertainties in $\mathrm{HC}$ and $\mathrm{mm} 2$.

2. $N_{\mathrm{T}}$ (molecular column density): in Sect. 4.2, we first assume that all the identified molecular lines are optically thin. Then, we use Eq. (10) to obtain the optical depth of those main isotopologue lines whose rare isotopologue lines with the same transition are detected. Finally, using Eq. (9), we correct the column density of the main isotopologue. In this approach, by assuming the rare isotopologue lines are optically thin, we list the optical depths and abundance ratios for ${ }^{13} \mathrm{CO}(2 \rightarrow 1)$, $\mathrm{CO}(2 \rightarrow 1)$, OCS $(19 \rightarrow 18)$, and $\mathrm{CH}_{3} \mathrm{CN}\left(12_{2} \rightarrow 11_{2}\right)$ with respect to the corresponding rare isotopologue lines with and without optical depth correction in Table 4.

However, Eq. (9) is based on the assumption that the main and rare isotopologue lines have the same excitation temperature, which is often not the case. Therefore, a more accurate calculation requires the large velocity gradient (LVG) approach and proper chemical modelling. Nevertheless, our analytic calculations suggest that, the optical depth is the main situation driving the error in the estimated molecular column density. For abundant molecules such as $\mathrm{CH}_{3} \mathrm{OH}, \mathrm{HNCO}$, $\mathrm{SO}$, and $\mathrm{SO}_{2}$, where we do not observe a corresponding transition of their rare isotopologues, our assumption that their lines are optically thin may result in underestimating their column densities by a factor of 7-10 (coming from the average differences for $\mathrm{OCS}, \mathrm{CO}$, and $\mathrm{CH}_{3} \mathrm{CN}$ column density values before and after optical depth correction in Fig. 10). Aside from the optical depth, the errors also come from our assumption of LTE. Assuming that the transitions of one species have ideal Boltzmann distributions at the same $T_{\text {rot }}$, molecular total column density can be derived from one transition. However, as represented by points on their rotation diagrams, multiple transitions of certain species (e.g., ${ }^{34} \mathrm{SO}_{2}$, $\mathrm{HNCO}$, and $\mathrm{CH}_{3} \mathrm{CH}_{2} \mathrm{OH}$, Fig. 9) show wide scatter, indicating that uncertainties in the estimation of $\mathrm{T}_{\text {rot }}$ lead to the over-/under-estimation of the total column densities by a factor of 0.5-2 (bold face in Tables A.5I,-A.7I). For the species whose total column densities are not able to be estimated from rotation diagram fittings owing to the lack of sufficient transitions, our LTE assumption (using Eq. (4)) may also result in the under/over-estimation of their total column densities of the same magnitude.

In addition, $\int T_{\mathrm{B}}(v) \mathrm{d} v$ measured from Gaussian/HFS fittings brings on average $<10 \%$ uncertainties (Table A.4), which is negligible compared to the chemical differentiation amongst the substructures (written as the subscript in Tables A.5I-A.7I).

3. $N_{\mathrm{H}_{2}}$ and molecular abundance: because no continuum information is available from the combined data, we used two methods to calculate the column density of $\mathrm{H}_{2}$ (Table 1): from SMA-only continuum $\left(N_{\mathrm{H}_{2}, 1}\right)$ and from SMA-30 m $\mathrm{C}^{18} \mathrm{O}\left(N_{\mathrm{H}_{2}, 2}\right)$. The molecular abundances were derived with respect to $\mathrm{H}_{2,2}$. Comparing the column density of $\mathrm{H}_{2,1}$ and 
S. Feng et al.: Resolving the chemical substructure of Orion-KL
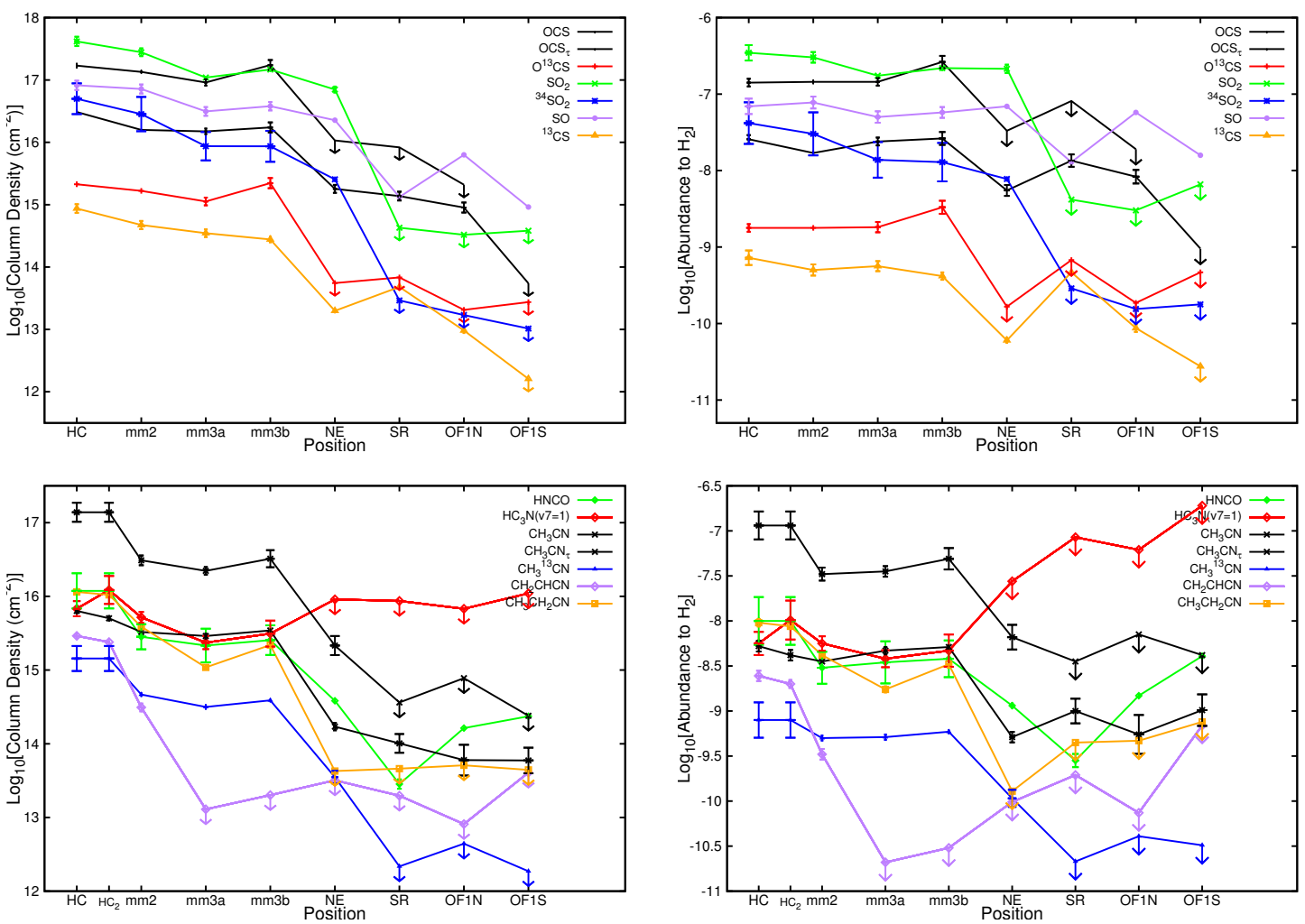

$* \mathrm{HC}_{7} \mathrm{~N}$ is not given because of large uncertainties of its tentative detection.
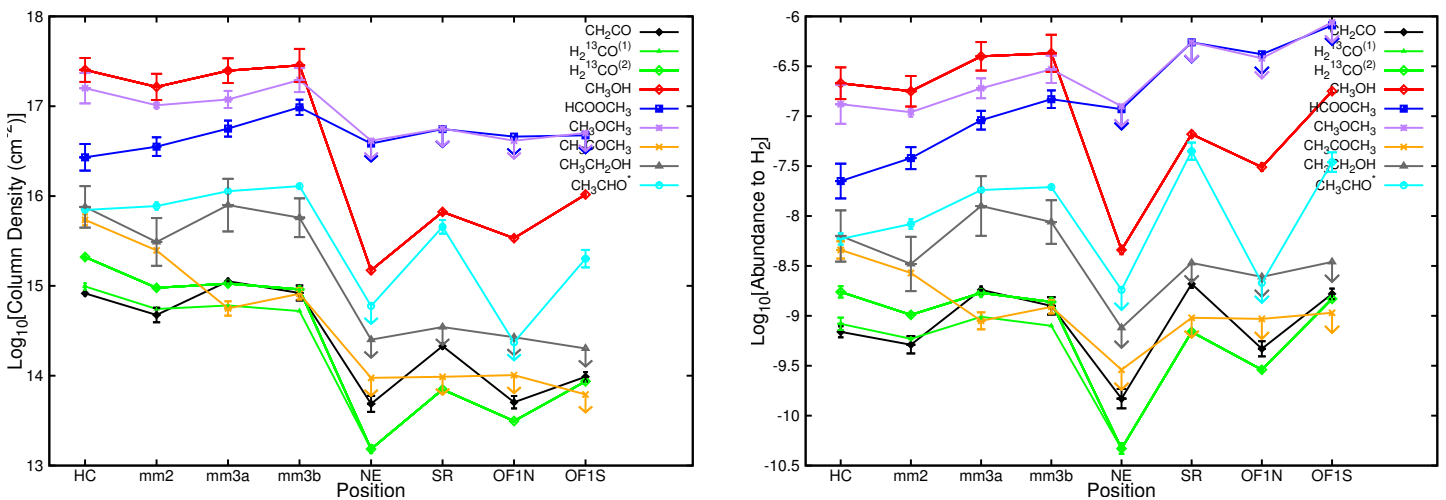

* Values of $\mathrm{H}_{2}^{13} \mathrm{CO}^{(1)}$ are derived from the temperatures the same as $\mathrm{HCOOCH}_{3}$; Values of $\mathrm{H}_{2}^{13} \mathrm{CO}^{(2)}$ are derived from the temperatures the same as $\mathrm{CH}_{3} \mathrm{OH}$.

$* \mathrm{C}_{6} \mathrm{H}$ is not given because of large uncertainties of its tentative detection.
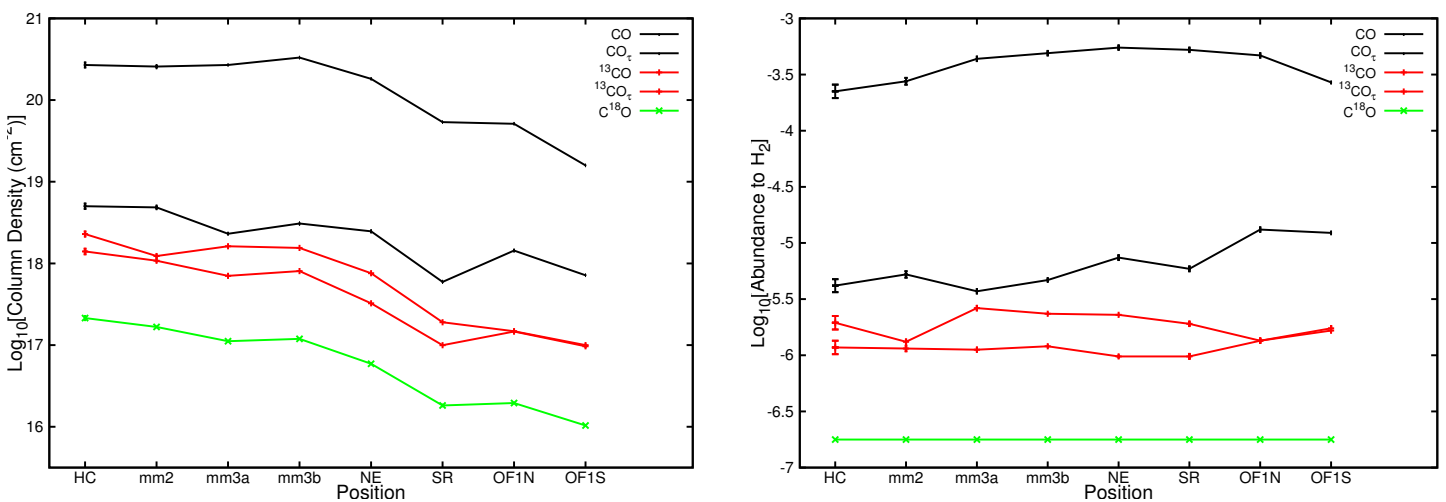

Fig. 10. Molecular column densities (panels in the left column) and abundances (panels in the right column) towards 8 positions in Orion-KL. The molecular abundances of each species with respect to $\mathrm{H}_{2}$ are converted from $\mathrm{C}^{18} \mathrm{O}$, assuming a constant $N_{\mathrm{C}^{18} \mathrm{O}} / N_{\mathrm{H}_{2}} \sim 1.79 \times 10^{-7}$ ratio. Column densities and abundances with the uncertainties are measured as mentioned in Sect. 4.4 and Tables A.5-A.7. Coloured solid lines show the differentiation for each isotopologue calculated from optically thin assumption (arrows highlight the upper limits), while dashed lines show the differentiation calculated with optical depth correction from Eq. (10). For abundant molecules (e.g., $\mathrm{HNCO}, \mathrm{SO}, \mathrm{SO}_{2}, \mathrm{CH}_{3} \mathrm{OH}$ ) which we have not measured their line optical depths, their densities could be underestimated by a factor of $7-10$. 
Table 4. Optical depth and abundance ratios for $\mathrm{CO},{ }^{13} \mathrm{CO}$, OCS, and $\mathrm{CH}_{3} \mathrm{CN}$ with respect to their rare isotopologues in each substructure of Orion KL.

\begin{tabular}{|c|c|c|c|c|c|c|c|c|c|c|}
\hline Species & & $\mathfrak{R}_{\alpha}$ & $\mathrm{HC}$ & $\mathrm{mm} 2$ & $\mathrm{~mm} 3 \mathrm{a}$ & $\mathrm{mm} 3 \mathrm{~b}$ & $\mathrm{NE}$ & S & OF1N & OF1S \\
\hline$\tau_{\mathrm{CO}(2 \rightarrow 1)}$ & & & 54 & 53 & 117 & 107 & 74 & 90 & 36 & 22 \\
\hline $\mathrm{N}_{\mathrm{CO}_{\tau}} / \mathrm{N}_{\mathrm{C}^{18} \mathrm{O}}$ & $\dagger$ & $560 \pm 25$ & $1263.0_{ \pm 21.8}$ & $1541.0_{ \pm 57.1}$ & $2422.0_{ \pm 46.6}$ & $2765.0_{ \pm 72.3}$ & $3100.0_{ \pm 52.9}$ & $2934.0_{ \pm 18.8}$ & $2640.0_{ \pm 33.7}$ & $1517.0_{ \pm 29.8}$ \\
\hline $\mathrm{N}_{\mathrm{CO}} / \mathrm{N}_{\mathrm{C}^{18} \mathrm{O}}$ & $\star$ & & $23.4_{ \pm 0.4}$ & $29.1_{ \pm 1.1}$ & $20.7_{ \pm 0.4}$ & $25.8_{ \pm 0.7}$ & $41.9_{ \pm 0.7}$ & $32.6_{ \pm 0.2}$ & $73.3_{ \pm 0.9}$ & $68.9_{ \pm 1.4}$ \\
\hline$\tau_{13} \mathrm{CO}(2 \rightarrow 1)$ & & & 1.1 & 0.25 & 2 & 1.5 & 2 & 1.5 & 0.02 & 0.08 \\
\hline $\mathrm{N}_{13} \mathrm{CO}_{\tau} / \mathrm{N}_{\mathrm{C}^{18} \mathrm{O}}$ & $\dagger$ & $7.2 \pm 0.9$ & $10.8_{ \pm 0.2}$ & $7.3_{ \pm 0.2}$ & $14.6_{ \pm 0.2}$ & $13.1_{ \pm 0.2}$ & $12.8_{ \pm 0.0}$ & $10.6_{ \pm 0.0}$ & $7.6_{ \pm 0.0}$ & $9.7_{ \pm 0.3}$ \\
\hline $\mathrm{N}_{{ }^{13} \mathrm{CO}} / \mathrm{N}_{\mathrm{C}^{18} \mathrm{O}}$ & 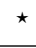 & & $6.5_{ \pm 0.1}$ & $6.5_{ \pm 0.2}$ & $6.3_{ \pm 0.1}$ & $6.8_{ \pm 0.1}$ & $5.5_{ \pm 0.0}$ & $5.5_{ \pm 0.0}$ & $7.5_{ \pm 0.0}$ & $9.3_{ \pm 0.3}$ \\
\hline$\tau_{\text {OCS }(19 \rightarrow 18)}$ & & & 5.5 & 8.5 & 6 & 10 & $\leq 6$ & $\leq 6$ & $\leq 2$ & - \\
\hline $\mathrm{N}_{\mathrm{OCS}_{\tau}} / \mathrm{N}_{\mathrm{O}^{13} \mathrm{CS}}$ & $\dagger$ & $77 \pm 7$ & $79.7_{ \pm 0.7}$ & $81.1_{ \pm 2.0}$ & $80.1_{ \pm 3.0}$ & $78.2_{ \pm 0.0}$ & $\leq 195.2$ & $\leq 121.3$ & $\leq 101.1$ & - \\
\hline $\mathrm{N}_{\mathrm{OCS}} / \mathrm{N}_{\mathrm{O}^{13} \mathrm{CS}}$ & $\star$ & & $14.4_{ \pm 0.1}$ & $9.5_{ \pm 0.2}$ & $13.3_{ \pm 0.5}$ & $7.8_{ \pm 0.0}$ & $\geq 32.4$ & $\geq 20.2$ & $\geq 43.7$ & - \\
\hline$\tau_{\mathrm{CH}_{3} \mathrm{CN}\left(12_{2} \rightarrow 11_{2}\right)}$ & & & 18 & 11 & 8.5 & 9 & 17 & $\leq 3.5$ & $\leq 13$ & $\leq 4$ \\
\hline $\mathrm{N}_{\mathrm{CH}_{3} \mathrm{CN}_{\tau}} / \mathrm{N}_{\mathrm{CH}_{3}^{13} \mathrm{CN}}$ & $\dagger$ & $77 \pm 7$ & $79.6_{ \pm 3.0}\left(76.6_{ \pm 2.4}\right)^{*}$ & $77.7_{ \pm 3.1}$ & $78.1_{ \pm 1.9}$ & $79.8_{ \pm 0.3}$ & $82.3_{ \pm 6.7}$ & $\leq 168.3$ & $\leq 176.3$ & $\leq 130.0$ \\
\hline $\mathrm{N}_{\mathrm{CH}_{3} \mathrm{CN}} / \mathrm{N}_{\mathrm{CH}_{3}^{13} \mathrm{CN}}$ & * & & $4.4_{ \pm 0.2}\left(4.3_{ \pm 0.1}\right)^{*}$ & $7.1_{ \pm 0.3}$ & $9.2_{ \pm 0.2}$ & $8.9_{ \pm 0.0}$ & $4.8_{ \pm 0.4}$ & $\geq 46.6$ & $\geq 13.6$ & $\geq 31.9$ \\
\hline
\end{tabular}

Notes. The values are derived from the single strongest transition of each species using Eqs. (3) and (4). $\dagger$ or $\star$ mark the estimations with or without optical depth correction. " $\geq(\leq)$ " come from the $3 \sigma$ limit of the non-detection of $\mathrm{O}^{13} \mathrm{CS}$ (OCS) and $\mathrm{CH}_{3}^{13} \mathrm{CN}$. The ratios between isotopes $\mathfrak{R}_{\alpha}$ of ${ }^{12} \mathrm{C} /{ }^{13} \mathrm{C}$ and ${ }^{16} \mathrm{O} /{ }^{18} \mathrm{O}$ are provided from the local ISM ratio measured in Wilson \& Rood (1994). Uncertainties on the measured values are typically $\leq 10 \%$ (written as the subscript), as determined from $T_{\text {rot }}$, partition function $Q\left(T_{\text {rot }}\right.$ ), and $\int T_{\mathrm{B}}(v) \mathrm{d} v$ of the transition. "**" denotes the ratio temperature derived from $\mathrm{CH}_{3}^{13} \mathrm{CN}$.

$\mathrm{H}_{2,2}$, we found $N_{\mathrm{H}_{2}, 2} / N_{\mathrm{H}_{2}, 1}$ is $\sim 0.9$ in $\mathrm{mm} 2, \sim 0.5$ in $\mathrm{mm} 3 \mathrm{a}$ and $\mathrm{mm} 3 \mathrm{~b}, \sim 0.4$ in $\mathrm{HC}^{22}, \sim 0.2$ in NE, but 0.1 in SR. Several reasons may lead to such difference: (1) the specific intensity $\left(\mathrm{Jy} \mathrm{beam}^{-1}\right)$ used to calculate the column density depends on the beam. The combined beam is larger than the SMA-only beam by a factor of $\sim 2.5$, so it is reasonable that for larger beam size, we obtain lower $\mathrm{H}_{2}$ column density (similar case in Beuther et al. 2007a). (2) In producing the continuum, we select the "line-free" part, but there are still lines with low emission (Fig. A.2), which may contaminate the continuum; (3) we simply assume that the gas-to-dust ratio and the volume densities in all substructures are similar within an order of magnitude $\left(\sim 150, \sim 10^{7} \mathrm{~cm}^{-3}\right)$, hence also the dust opacity. Since we do not know the size of each substructure, gas is possibly much denser in a warmer source (HC, mm2, mm3a, and $\mathrm{mm} 3 \mathrm{~b}$ ) than in the other cooler substructures (NE, SR), so the homogeneous volume density assumption may not be accurate in the whole region. (4) Grain growth in the envelopes can also be a reason for difference in dust emissivity from centre to extended substructures at (sub-)mm (Miotello et al. 2014). (5) We also note that $\mathrm{C}^{18} \mathrm{O}$ column density in the central region is a factor of 30 higher than in the outflow. Judging that $N_{\mathrm{H}_{2}, 2}$ is derived with the assumption that $N_{\mathrm{C}^{18} \mathrm{O}} / N_{\mathrm{H}_{2}}=$ const. in all the studied substructures, the variation of $N_{\mathrm{C}^{18} \mathrm{O}} / N_{\mathrm{H}_{2}}$ ratio in different substructures can be another factor that leads to error in our estimation.

4. In addition, another simplification in our calculation is an assumed constant beam-filing factor. Determination of molecular abundances in Orion-KL substructures relies upon an accurate source structure model for each molecule. When the source is smaller than the beam size, the main beam brightness temperature is diluted, leading to an underestimated brightness temperature for more compact structures. To correct this, an appropriate correction factor needs to be applied. Since we do not know the accurate sizes of the substructures, we simply assume that the filling factor is unity in all the calculations.

22 In the extreme case, the ratio is $0.8-1$, if assuming the ice mantle is completely evaporated.

\section{Discussion}

These observations comprise a large set of molecular lines (over 160 lines from 20 species, including 25 isotopologues) from combined SMA and IRAM $30 \mathrm{~m}$ data towards Orion-KL. Resolving Orion-KL into eight substructures and comparing S-, N-, and O-bearing molecules, we find complicated spatial morphologies for COMs and strong chemical differentiation over the observed substructures.

\subsection{Comparison with the other results}

Previous quantitative studies of Orion-KL mainly focused on the $\mathrm{HC}, \mathrm{CR}$ (with mm3a and $\mathrm{mm} 3 \mathrm{~b}$ treated as a single substructure), and plateau (OF1N, OF1S), therefore comparison of our estimated molecular abundances with previous results is limited to these substructures.

1. Since different molecules have different chemistry, the rotation temperatures derived using different tracers in the same substructure vary. Comparing with the results of others studies, the variation in $T_{\text {rot }}$ for different species can be explained.

- Our opacity-corrected temperature in HC derived from $\mathrm{CH}_{3} \mathrm{CN}$ is $120-160 \mathrm{~K}$, which is a factor of 1.5 lower than reported by Goddi et al. (2011a) from $\mathrm{NH}_{3}$ at a higher angular resolution $\left(\sim 0.8^{\prime \prime}\right)$. Given that $\mathrm{HNCO}$ in our dataset probes the similar temperatures, our slightly lower temperatures for $\mathrm{N}$-bearing molecules may trace the envelope region of the $\mathrm{HC}$.

- Our adopted temperatures from $\mathrm{HCOOCH}_{3}$ agree with the temperatures estimated by Favre et al. (2011a) from observations of this species at similar spatial resolution $^{23}$. However, they are lower than those reported in Brouillet et al. (2013) owing to our spectral

${ }^{23}$ MF1-5 are the $\mathrm{HCOOCH}_{3}$ emission peaks in Favre et al. (2011a), which coincide with mm3b (MF1), HC (MF2), mm3a (MF3), and mm2 (MF4-5) in our continuum. 
resolution. Multiplets of $\mathrm{CH}_{3} \mathrm{OCH}_{3}$ and $\mathrm{CH}_{3} \mathrm{CH}_{2} \mathrm{OH}$ are not resolved in this paper, so we treat each detected line of these species as a single component, and temperatures obtained from rotation diagrams are potentially underestimated $^{24}$.

- Temperatures probed by $\mathrm{CH}_{3} \mathrm{OH}(>200 \mathrm{~K}$ in $\mathrm{HC}, \sim 170$ in $\mathrm{mm} 2$, and $\sim 160 \mathrm{~K}$ in $\mathrm{CR}$ ) are higher than those probed by the other molecules, probably because of the lack of optical depth corrections, or because they trace gas with different temperatures (discussed in Sect. 4.4 part 1 (2)). Nevertheless, these temperatures agree with those derived from the same species by Sutton et al. (1995) $\left({ }^{13} \mathrm{CH}_{3} \mathrm{OH}\right)$ and Friedel \& Widicus Weaver (2012) in HC and CR, and by Beuther et al. (2005) in $\mathrm{HC}$ and $\mathrm{mm} 2$.

- We note that S-bearing species may be affected by shocks in the environment. Although they were not often derived previously, and the high $E_{\mathrm{u}} / k_{\mathrm{B}}$ transition leads to large uncertainties in the rotation temperatures derived from ${ }^{34} \mathrm{SO}_{2}$, our estimated temperatures are within the uncertainty of those derived by Esplugues et al. (2013) from $\mathrm{SO}_{2}$ in $\mathrm{HC}(190 \pm 60 \mathrm{~K})$ and $\mathrm{CR}(80 \pm 30 \mathrm{~K})$.

2. Due to the compactness of the detected substructures, column densities of $\mathrm{CO},{ }^{13} \mathrm{CO}$, and $\mathrm{C}^{18} \mathrm{O}$ are ten times higher than those from lower resolution observations in single-dish observations of Wilson et al. (2011) $\left(F W H P \sim 13^{\prime \prime}\right)$ and in Plume et al. (2012) (Herschel/HIFI beam ranges from 13" to $\left.40^{\prime \prime}\right)$. If taking the beam ratio into account, our results are consistent with previous measurement.

3. In both $\mathrm{HC}$ and $\mathrm{CR}$, the abundances of $\mathrm{SO}, \mathrm{SO}_{2}, \mathrm{HNCO}$, $\mathrm{HC}_{3} \mathrm{~N}, \mathrm{CH}_{3} \mathrm{OH}, \mathrm{CH}_{2} \mathrm{CHCN}, \mathrm{CH}_{3} \mathrm{CH}_{2} \mathrm{CN}$, and $\mathrm{CO}$ are comparable to the values in Blake et al. (1987), Wright et al. (1996), and Esplugues et al. (2013), within our factor of 1.5-2 uncertainties. $\mathrm{H}_{2}^{13} \mathrm{CO}$ is lower than the value derived from $\mathrm{H}_{2} \mathrm{CO}$ in Blake et al. (1987), assuming a standard ${ }^{12} \mathrm{C} /{ }^{13} \mathrm{C}$ ratio, but is comparable to the value in Wright et al. (1996). After the optical depth corrections, our OCS and $\mathrm{CH}_{3} \mathrm{CN}$ abundances are ten times higher than those in Blake et al. (1987), because the latter estimation was made assuming lines of these species to be optically thin. However, our abundances of OCS and ${ }^{13} \mathrm{CS}$ are comparable to those in Tercero et al. (2010), within our factor of 2 uncertainty.

4. Similar to the gradient in Sutton et al. (1995) and Wright et al. (1996), CO and SO have stronger emission and higher abundances in the plateau of both the highand low-velocity outflows, which has been suggested to be due to shock enhancement in these regions. Although molecules in general have around 1-2 mag lower abundances in our low-velocity outflow than the values reported in the NE-SW plateau from previous interferometric study (Wright et al. 1996), their abundances in our high-velocity outflow are comparable to the single dish results in the NW-SE plateau (Sutton et al. 1995).

\footnotetext{
24 Brouillet et al. (2013) resolves the $\mathrm{CH}_{3} \mathrm{OCH}_{3}$ multiplets and uses them to estimate temperatures as well as column densities. However, if each multiplet is treated as a single component, it would derive temperatures consistent with those we estimate.
}

5. The estimated column densities of COMs $\left(\mathrm{CH}_{3} \mathrm{CH}_{2} \mathrm{CN}\right.$, $\mathrm{CH}_{3} \mathrm{COCH}_{3}, \mathrm{CH}_{3} \mathrm{OCH}_{3}$, and $\mathrm{HCOOCH}_{3}$ ) in $\mathrm{HC}$ are found to be lower than the values in Friedel \& Snyder (2008) by a factor of 10 , because their observations have a smaller synthetic beam. However, $\mathrm{HCOOCH}_{3}$ has comparable column densities to Favre et al. (2011a) in mm2 (MF4-5), mm3a (MF3), and mm3b (MF1), as does $\mathrm{CH}_{3} \mathrm{OCH}_{3}$ compared to Brouillet et al. (2013) at all the central condensations (HC, $\mathrm{mm} 2, \mathrm{~mm} 3 \mathrm{a}$, and $\mathrm{mm} 3 \mathrm{~b}$ ), and the abundance of $\mathrm{CH}_{3} \mathrm{COCH}_{3}$ compared to Peng et al. (2013) at HC and mm2 (Ace-3).

To sum up, the abundance of most species from our study are comparable to previous single-dish studies especially in the central condensations. Therefore, molecular abundance estimation from single-dish observations for the farther sources (e.g., ten times farther than Orion-KL) should be trustworthy at least in the source centre after optical depth correction.

\subsection{Chemistry in the hot molecular core}

At a spatial resolution of $\sim 1200 \mathrm{AU}$, our data resolves Orion-KL into several condensations. The specific intensities $\left(\mathrm{Jy}_{\mathrm{beam}}{ }^{-1}\right)$ of the dust continuum peaks exhibit a gradient from the central condensations to the extended structures, and the gas temperatures exhibit the same gradient: The HC is the strongest continuum substructure, because it has both the highest gas temperature and column density; $\mathrm{mm} 2, \mathrm{~mm} 3 \mathrm{a}$, and $\mathrm{mm} 3 \mathrm{~b}$ are slightly fainter and less dense; while NE and SR have weaker continuum and line emission than the former sources, but stronger emission than $\mathrm{OF} 1 \mathrm{~N}(\mathrm{~S})$.

Differentiation in the spatial distribution of $\mathrm{S}-, \mathrm{N}-$, and O-bearing species clearly shows that chemistry in the central condensations of Orion-KL is not homogeneous. Simple $\mathrm{N}$ - and O-bearing molecules show the strongest emission towards the central condensations. However, unlike most of the $\mathrm{N}$-bearing species (including $\mathrm{N}$-bearing COMs) which are not detected in SR, NE, or the outflow, simple organic molecules $\left(\mathrm{H}_{2}^{13} \mathrm{CO}, \mathrm{CH}_{2} \mathrm{CO}\right)$ and $\mathrm{CO}\left({ }^{13} \mathrm{CO}, \mathrm{C}^{18} \mathrm{O}\right)$ have bright extended emission even towards the cooler and less dense regions (SR, OF1N, and OF1S). Compared to the above extreme cases, the S-bearing molecules are widely distributed over the central condensations, NE and (or) SR. A possible reason for this is that the S-bearing molecules are mainly formed via warm gasphase chemistry or shock chemistry (e.g., Pineau des Forets et al. 1993; Charnley et al. 1997), and then coincide with both $\mathrm{N}$ and O-bearing molecules in the plateau (NE) of BN/KL (e.g., Chandler \& Wood 1997; Schreyer \& Henning 1999, see discussion in Sect. 5.3).

For the COMs, it has been widely suggested that "N-/Ochemical differentiation" can be caused by lower or higher temperatures during the cold pre-hot molecular core phase and different paces of warm-up in these regions (Caselli et al. 1993; Garrod et al. 2008; Neill et al. 2011; Laas et al. 2011). Besides confirming the $\mathrm{HC}$ peak of $\mathrm{N}$-bearing $\mathrm{COMs}\left(\mathrm{CH}_{2} \mathrm{CHCN}\right.$, $\mathrm{CH}_{3} \mathrm{CH}_{2} \mathrm{CN}, \mathrm{CH}_{3} \mathrm{CN}$, and $\mathrm{CH}_{3}^{13} \mathrm{CN}$ ) and the mm3a (or/and $\mathrm{mm} 3 \mathrm{~b}$ ) peak of several O-bearing COMs (e.g., $\mathrm{HCOOCH}_{3}$ and $\mathrm{CH}_{3} \mathrm{OCH}_{3}$ ), we found several other COMs which cannot be classified within these dichotomous emission peaks groups (also see Guélin et al. 2008; Friedel \& Widicus Weaver 2012; Peng et al. 2013). For example, the peak of $\mathrm{CH}_{3} \mathrm{OH}$ is velocity dependent, shifting from the $\mathrm{HC}\left(3.6 \mathrm{~km} \mathrm{~s}^{-1}\right)$ through $\mathrm{mm} 3 \mathrm{a}\left(6 \mathrm{~km} \mathrm{~s}^{-1}\right)$ to $\mathrm{mm} 3 \mathrm{~b}\left(7.2 \mathrm{~km} \mathrm{~s}^{-1}\right)$; the peak of $\mathrm{CH}_{3} \mathrm{CH}_{2} \mathrm{OH}$ is in between the $\mathrm{HC}$ and $\mathrm{mm} 3 \mathrm{a}$. In contrast, $\mathrm{CH}_{3} \mathrm{COCH}_{3}$ shares a morphology with $\mathrm{N}$-bearing molecules-peaking towards the $\mathrm{HC}$ with 
a V-shaped distribution. This similarity in morphologies may indicate a link between the HC-peaking N-bearing species and the $\mathrm{mm} 3 \mathrm{a}$ (or/and $\mathrm{mm} 3 \mathrm{~b}$ )-peaking species, therefore their formation pathways likely involve $\mathrm{NH}_{3}$ (ammonia) or another major N-bearing molecule (e.g., Chen \& Woon 2011), or need similar physical conditions appropriate to their production and/or sublimation, such as shocks (Peng et al. 2013). Moreover, although $\mathrm{CH}_{3} \mathrm{CHO}$ is only tentatively detected, it peaks in a similar location to $\mathrm{HCOOCH}_{3}$. Furthermore, like $\mathrm{CH}_{2} \mathrm{CO}$, it exhibits extended emission from the NE to the SE tail, suggesting these three species may share a common or at least a related formation path.

The favoured model of hot molecular core chemistry so far is the gas-grain model, where frozen radicals, partly produced by photolysis, become mobile during the warm-up phase, and their reactions produce COMs (Markwick et al. 2000; Garrod et al. 2008). Three phases are usually modelled, the cold, warm-up, and hot molecular core stages. During these stages, different species are defined as zeroth-generation (e.g., $\mathrm{CH}_{3} \mathrm{OH}$ ), firstgeneration (e.g., $\mathrm{HCOOCH}_{3}$ ), and second-generation organic molecules (see Herbst \& van Dishoeck 2009). In such a scenario, since the critical densities are fairly similar for $\mathrm{N}$ - and O-bearing species, the distribution diversity of species in OrionKL may also be explained by different initial compositions or different chemical "ages" (evolutionary stage, the time that the gas phase species has had to evolve from an initial composition after being released from ice mantles, Friedel \& Snyder 2008). If that is the case, $\mathrm{COMs}$ like $\mathrm{CH}_{3} \mathrm{OCH}_{3} / \mathrm{CH}_{3} \mathrm{OH}$ can act as chemical clocks, implying that the different substructures in BN/KL could have different chemical ages (e.g., Charnley et al. 1997; Wakelam et al. 2004).

In addition to grain-surface and gas-phase chemical processing, it is also possible that varying physical conditions, including temperature, density, and kinematics cause the differentiation in the spatial distributions of molecular line emission seen in the Orion-KL region. Probably due to the difference in the central protostars, the warm-up paces of different substructures vary. The modelling result from Garrod et al. (2008, especially their Fig. 8) is an example. In a particular substructure during the warm-up process (e.g., from $100 \mathrm{~K}$ to $200 \mathrm{~K}$ ), the abundances of some molecules increase (e.g., $\mathrm{HNCO}, \mathrm{CH}_{3} \mathrm{CN}$, $\mathrm{CH}_{3} \mathrm{OH}$ ) because of their evaporation from the grain surface to the gas phase, while the abundances of some large molecules cannot be synthesized on grains any longer because their precursor species have desorbed to the gas phase. They decrease (e.g., $\left.\mathrm{HCOOCH}_{3}, \mathrm{CH}_{3} \mathrm{OCH}_{3}\right)$ or start to decrease $\left(\mathrm{CH}_{3} \mathrm{OH}\right)$. Another possibility is that these species are destroyed by ion-molecule chemistry (e.g., by protonation via $\mathrm{XH}^{+}$molecules), followed by dissociative recombination into multiple small fragments. In this scenario, the different emission peaks of different species can be explained by the higher temperature in HC $(120-160 \mathrm{~K})$ than in $\mathrm{mm} 3 \mathrm{a}$ and $\mathrm{mm} 3 \mathrm{~b}(80-100 \mathrm{~K})$, meaning that $\mathrm{N}$-bearing species are still evaporating in $\mathrm{HC}$ but formation of molecules such as $\mathrm{HCOOCH}_{3}$ is stopped or it is dissociating there.

\subsection{Chemistry in the outflow}

Outflows from young stars can destroy grain material and/or liberate ices when they impact the surrounding cold matter, meaning that chemical reactions can be driven by the associated shocks even in cold gas (Herbst \& van Dishoeck 2009). For years, the outflows in Orion-KL have been the main subject of chemical studies of this region (Wright et al. 1996 and references therein). Although COMs are less abundant or not detected in these outflows, the chemistry of simple molecules especially shock tracers can provide an alternative way to investigate the gas structure of the outflows.

Two outflows have been reported in Orion-KL, almost perpendicular to each other. In addition, along the NE-SW direction, a large-scale "integral-shaped" filament has been detected aligned with the low-velocity outflow (Wright et al. 1992; Lis et al. 1998; Di Francesco et al. 2008). To confirm whether the NE clump is associated with the filament or the outflow, we selected several widespread species, and use a Gaussian function to fit their line profile extracted from NE (Fig. 7). Broad line wings $\left(\mathrm{C}^{18} \mathrm{O}\right)$ and a second component $\left(\mathrm{HNCO}, \mathrm{SO}_{2}\right.$, and ${ }^{34} \mathrm{SO}$ ) with $V_{\text {peak }} \sim 20-23 \mathrm{~km} \mathrm{~s}^{-1}$ indicate that line emission in $\mathrm{NE}$ contains significant contribution from the outflow and is part of the plateau.

To study the chemistry in the outflows, we image the blueand red-shifted gas of several shock-tracing molecules along the outflow direction (Fig. A.4). To avoid contamination from blending, we image as broad a velocity range as possible (labelled in each panel), though, $-20 \sim+30 \mathrm{~km} \mathrm{~s}^{-1}$ is the limit for most lines other than SO, CO, and ${ }^{13} \mathrm{CO}$. Because of the range limit and the limits of the $52^{\prime \prime}$ primary beam, the highvelocity outflow (30-100 $\mathrm{km} \mathrm{s}^{-1}$ ) cannot be imaged in its entirety. However, the low-velocity outflow $\left(\sim 18 \mathrm{~km} \mathrm{~s}^{-1}\right)$ with a NE-SW elongated structure is clearly detected, showing bipolar structure in the intensity-integrated map of $\mathrm{H}_{2}^{13} \mathrm{CO}$. In addition, intensity-integrated maps of such molecules as OCS, ${ }^{34} \mathrm{SO}_{2}, \mathrm{SO}, \mathrm{HNCO}, \mathrm{CH}_{2} \mathrm{CO}, \mathrm{CO}$, and ${ }^{13} \mathrm{CO}$ all have a "butterfly" morphology, with blue-shifted radial velocities of the moving gas towards the NW and the red-shifted velocities in the SE. This morphology is exactly the same as found in SiO (Wright et al. 1995; Plambeck et al. 2009; Zapata et al. 2012; Niederhofer et al. 2012) and $\mathrm{H}_{2} \mathrm{O}$ maser observations (Greenhill et al. 2013) on scales of tens of AU. Whether this morphology is due to the bipolar lobes of the high-velocity outflow or to the expanding low-velocity outflow is still not clear. Possibilities are discussed (e.g., two bipolar outflows from a precessing binary, ballistic ejecta from a rotating disk, a single expanding wide-angle NE-SW outflow; Zapata et al. 2012). The asymmetry shape of the lobes (e.g., "U" shape blue-shifted and half "U" red-shifted lobes in the map of SO) is said to come from some density inhomogeneities in the surroundings (Zapata et al. 2012) or the interaction between outflow and the environment (Niederhofer et al. 2012).

Therefore, it can be suggested that a picture of explosive NW-SE outflow explains the excitation temperature gradient from the central condensations to the extended outflows in Orion-KL (Zapata et al. 2012): the decelerated bullets can be absorbed by the BN/KL cloud, and their kinetic energy can be transferred to thermal energy (Peng et al. 2012b); the closer a gas parcel is to the explosion centre (e.g., the closer encounter of BN, source I and maybe also source N, suggested by proper motion studies from Rodríguez et al. 2005; Gómez et al. 2005; 2008 and 3D modelling from Nissen et al. 2012), the higher the thermal energy available to affect the chemical properties of the gas in the $\mathrm{HC}$ and $\mathrm{mm} 2$.

Of all the shock-related molecules, S-bearing species are of special interest, not only because their abundance may be enhanced by shocks (Martin-Pintado et al. 1992; Pineau des Forets et al. 1993; Chernin et al. 1994; Bachiller \& Pérez 1997; Schilke et al. 1997; Charnley et al. 1997; Bachiller et al. 2001; Viti et al. 2004; Garrod 2013), but also because they have been proposed as potential chemical clocks to date outflows (and hence their protostellar driving source; 
e.g., Charnley et al. 1997; Wakelam et al. 2004). The angular resolution of our study does not allow us to investigate the post/pre-shocked chemistry. In spite of that, we do find that some S-bearing molecules (e.g., $\mathrm{SO}_{2},{ }^{34} \mathrm{SO}_{2}$, and $\mathrm{SO}$ ) have almost the same abundances in NE as in the central sources, which are higher than those in $\mathrm{OF} 1 \mathrm{~N}(\mathrm{~S})$, even though we assume the temperatures in $\mathrm{NE}$ and $\mathrm{OF} 1 \mathrm{~N}(\mathrm{~S})$ are equally low. The enhancement of their abundance may indicate stronger shocks in this direction than the rest of Orion-KL. On the other hand, although we cannot tell the precise ages of different substructures, we can compare the variations in abundance ratios $\mathrm{SO}_{2} / \mathrm{SO}$ in our observations to the gas-grain modelling result. To simplify the case, we assume that the temperatures in all substructures are $100 \mathrm{~K}$ and that all substructures have the same density $\sim 10^{7} \mathrm{~cm}^{-3}$; and we estimate the value of $\mathrm{SO}_{2}$ (lines are possibly optically thick) by using ${ }^{34} \mathrm{SO}_{2}$ and a ratio of ${ }^{32} \mathrm{~S} /{ }^{34} \mathrm{~S}=22$ (Wilson \& Rood 1994). Comparing the observed $\mathrm{SO}_{2} / \mathrm{SO}$ ratio to modelling of Wakelam et al. $(2004)^{25}$ in all substructures (Fig. 11), we find that the HC has the highest $\mathrm{SO}_{2} / \mathrm{SO}$ ratio, which suggests it is the most evolved substructure. Also, $\mathrm{mm} 2, \mathrm{~mm} 3 \mathrm{a}$, and $\mathrm{mm} 3 \mathrm{~b}$ have lower ratios and are younger. The lowest ratios are in the outflows, which indicate the age of the low-velocity outflow should be $\ll 1 \times 10^{4}$ years, while the high-velocity outflow should be $\ll 1 \times 10^{3}$ years $^{26}$.

The shocks could also be responsible for the observed chemical differentiation in COMs (e.g., by stripping ices from grains). The CR (together with $\mathrm{mm} 3 \mathrm{a}$ and $\mathrm{mm} 3 \mathrm{~b}$ ) is thought to be the place where icy grains are released into the gas phase by an outflow from the HC (Blake et al. 1987; Liu et al. 2002). Therefore, based on the reported association between the emissions of $\mathrm{HCOOCH}_{3}$ and the $2.12 \mu \mathrm{m}$ vibrationally excited $\mathrm{H}_{2}$ (Lacombe et al. 2004), it has been suggested that shocks between the ISM and high-velocity gas "bullets" from the outflow (Zapata et al. 2009; Bally et al. 2011) explains the production of COMs, which has the same morphology as $\mathrm{HCOOCH}_{3}$ (Favre et al. 2011b). In a similar scenario, N-bearing COMs may be released to the gas phase north-east of the $\mathrm{HC}$ (e.g., $\mathrm{HC}_{3} \mathrm{~N}, \mathrm{CH}_{2} \mathrm{CHCN}$ ), where the low-velocity outflow is impacting the ambient dusty material, and the weak emission /nondetection of these molecules along the high-velocity outflow may be due to lack of dusty material in the NW-SE direction (Friedel \& Widicus Weaver 2012).

\subsection{Search for COMs}

The chemistry of Orion-KL is rich, not only in high-density tracers and shock tracers, but also with numerous transitions of COMs, which are less abundant than the other tracers and which have a less well understood formation mechanism.

Three possibilities have been discussed for the formation of the COMs: (1) ion-molecule chemistry involving large radicals (Ehrenfreund \& Charnley 2000); (2) formation on dust grains and then released to the gas at higher temperatures in the later stages of star formation (Garrod et al. 2008; Herbst \& van Dishoeck 2009); (3) a hybrid approach that COMs

25 In Wakelam et al. (2004), $\mathrm{SO} / \mathrm{H}_{2}$ ratio is sensitive to the grain components. However, $\mathrm{SO}_{2} / \mathrm{SO}$ ratios from different grain component models are similar at a particular time (yr), so we compare this ratio to the observations.

${ }^{26} \mathrm{NE}$ is part of the filamentary structure, so the age of the low-velocity outflow is implied from the upper limit ratio in SR. Due to the nondetection of $\mathrm{SO}_{2}\left({ }^{34} \mathrm{SO}_{2}\right)$ in OF1N(S), the age of the high-velocity outflow is implied from the upper limit ratio in OF1N.
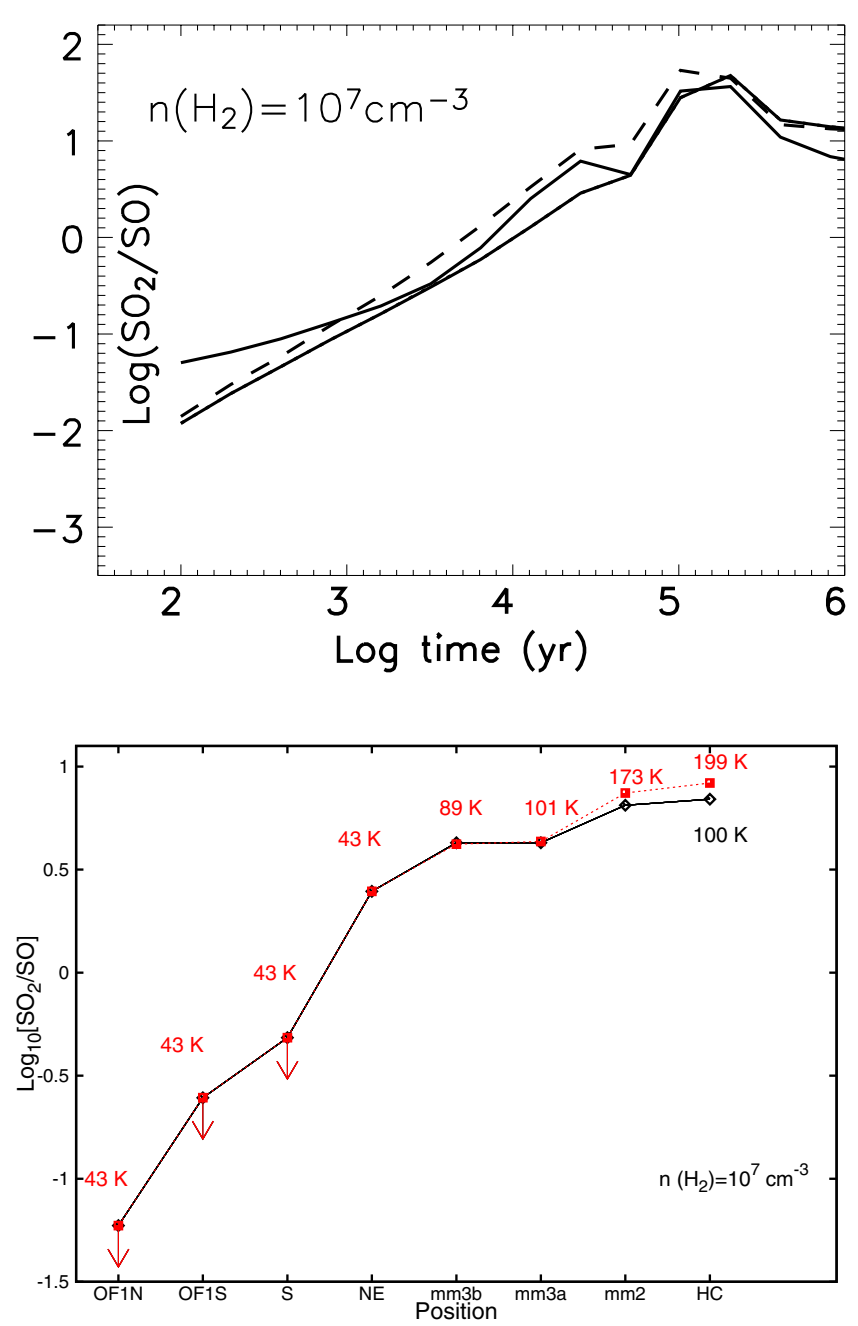

Fig. 11. Observed $\mathrm{SO}_{2} / \mathrm{SO}$ ratio compared to the model prediction. The upper panel is adopted from Fig. 5 of Wakelam et al. (2004) (100 K, $10^{7} \mathrm{~cm}^{-3}$ ), where different line types are from different grain component models. The lower panel is from our observed $\mathrm{SO}_{2} / \mathrm{SO}$ ratio by assuming (1) all substructures have similar density $10^{7} \mathrm{~cm}^{-3}$ and (2) $N_{\mathrm{SO}_{2}} / N_{34} \mathrm{SO}_{2}=22$ (Wilson \& Rood 1994). The black solid line comes from the assumption that all substructures have a temperature close to $100 \mathrm{~K}$, and the red dotted line comes from different temperatures derived in Sect. 4.1 (labelled in red).

are ejected into the gas via efficient reactive desorption, after a sequence of gas-phase reactions between precursor species formed on grain surfaces (Vasyunin \& Herbst 2013). In our dataset, we search for several COMs (Table 2) by using the synthetic fitting programme described in Sect. 3.2.2. $\mathrm{HC}_{7} \mathrm{~N}, \mathrm{C}_{6} \mathrm{H}$, and $\mathrm{CH}_{3} \mathrm{CHO}$ are tentatively detected because of missing predicted lines. For the remaining species, if not taking the blending problem into account, all the predicted transitions are robustly detected. In addition, we find that saturated molecules are likely to be more abundant than unsaturated molecules (e.g., $\mathrm{HC}_{3} \mathrm{~N}$, $\mathrm{C}_{6} \mathrm{H}, \mathrm{HC}_{7} \mathrm{~N}$ ). This indicates that these saturated species may be formed on dust grain surfaces by subsequent hydrogenation of their precursor species (such as $\mathrm{CO}, \mathrm{O}, \mathrm{N}$ or their more complex combinations).

Unfortunately, we lack the sensitivity to detect $\mathrm{HCOOH}$ (formic acid), which has been previously reported in OrionKL by Liu et al. (2002). Line blending also prevents us from robustly detecting $\mathrm{CH}_{2} \mathrm{OHCHO}$ (glycolaldehyde) and 
$\left(\mathrm{CH}_{2} \mathrm{OH}\right)_{2}$ (ethylene glycol) in our data. We also fail to detect other prebiotic molecules, e.g., $\mathrm{NH}_{2} \mathrm{CH}_{2} \mathrm{COOH}$ (glycine; Garrod 2013), $\mathrm{CH}_{3} \mathrm{COOH}$ (acetic acid), $\mathrm{NH}_{2} \mathrm{CH}_{2} \mathrm{CN}$ (amino acetonitrile; Belloche et al. 2008), $\mathrm{CH}_{3} \mathrm{CONH}_{2}$ (acetamide; van Dishoeck 2009), and the ring molecules. More sensitive observations (e.g., with ALMA) are required to determine whether these molecules exist in the Orion-KL environment.

\section{Conclusions}

Based on the analysis of combined SMA and $30 \mathrm{~m}$ observations of Orion-KL, we have drawn the following conclusions:

1. From interferometric observations with the SMA, Orion-KL has been resolved into several continuum substructures with different peak flux densities, at the angular resolution of $3^{\prime \prime}$ (1200 AU at a distance of $414 \mathrm{pc}$ ). In addition to the previous well-known compact continuum substructures like the HC and $\mathrm{mm} 2$, our data resolves the clump south-west of $\mathrm{HC}$ into two cores: $\mathrm{mm} 3 \mathrm{a}$ and $\mathrm{mm} 3 \mathrm{~b}$. In addition, we confirmed the detection of strong continuum emissions $(>5 \sigma)$ from two resolved clumps (SR and NE), which lie along the axis of both the low-velocity outflow and the large-scale dusty filament.

2. For the first time, we have combined interferometric and single-dish observations of Orion-KL, to correct missing short spacings, and achieved high sensitivity to all spatial scales. Covering a field of view of 52", we mapped the distribution of all the identified species. In particular, we obtained the first maps of molecules such ${ }^{34} \mathrm{SO}_{2}, \mathrm{O}^{13} \mathrm{CS}$, HNCO, $\mathrm{H}_{2}^{13} \mathrm{CO},{ }^{13} \mathrm{CO}$, and $\mathrm{CH}_{2} \mathrm{CO}$ with both high resolution and sensitivity to spatial information on all scales. We also identified several transitions of low abundance $\left(<10^{-8}\right.$ with respect to $\mathrm{H}_{2}$ ) $\mathrm{COMs}$, such as $\mathrm{CH}_{3} \mathrm{COCH}_{3}$ and $\mathrm{CH}_{3} \mathrm{CH}_{2} \mathrm{OH}$, as well as made the tentative detection of $\mathrm{CH}_{3} \mathrm{CHO}$ and long carbon chains like $\mathrm{C}_{6} \mathrm{H}$ and $\mathrm{HC}_{7} \mathrm{~N}$.

3. The Orion KL region exhibits clear chemical differentiation on the scales probed by the SMA- $30 \mathrm{~m}$ observations. The emission from S-bearing molecules, $\mathrm{CO}$ isotopologues, and simple organic molecules are extended, covering not only the central condensations ( $\mathrm{HC}, \mathrm{mm} 2, \mathrm{~mm} 3 \mathrm{a}$, and $\mathrm{mm} 3 \mathrm{~b}$ ), but also the NE clump, the SR, and even the outflow regions. In comparison, the distributions of COMs are more concentrated and hard to group. The segregated emission peaks at either the $\mathrm{HC}$ (e.g., N-bearing) or $\mathrm{CR}$ (together with mm3a and mm3b, e.g., $\mathrm{HCOOCH}_{3}, \mathrm{CH}_{3} \mathrm{OCH}_{3}$ ) indicates different formation pathways for O-bearing and $\mathrm{N}$ bearing molecules. However, some COMs peak in-between the $\mathrm{HC}$ and $\mathrm{mm} 3 \mathrm{a}\left(\mathrm{CH}_{3} \mathrm{CH}_{2} \mathrm{OH}\right)$, or have dual velocity dependent peaks $\left(\mathrm{CH}_{3} \mathrm{OH}\right)$, or share the same morphology as $\mathrm{N}$-bearing species $\left(\mathrm{CH}_{3} \mathrm{COCH} 3\right)$, which may indicate the linking of some formation pathways between O-bearing and $\mathrm{N}$-bearing molecules. Moreover, line widths of the Nbearing, S-bearing species, $\mathrm{HCOOCH}_{3}$ and $\mathrm{CH}_{3} \mathrm{OH}$ vary, and so do the derived rotation temperatures, indicating they trace different gas.

4. By studying the chemistry within outflows of Orion-KL, we found higher abundances of such shock tracers as ${ }^{34} \mathrm{SO}_{2}$, $\mathrm{SO}_{2}$, and $\mathrm{SO}$ in the plateau of the low-velocity outflow (NE), indicating stronger shocks in this direction than the rest of Orion-KL.

5. Molecular rotation temperatures and abundances show large gradience between central condensations and the outflow regions, indicating difference between hot molecular core and shock chemistry.
Acknowledgements. We would like to thank the SMA staff and IRAM $30 \mathrm{~m}$ staff for their helpful support during the reduction of the SMA data, the performance of the IRAM $30 \mathrm{~m}$ observations in service mode, Z. Y. Zhang and Y. Wang for helping with code development, for useful discussion, and the referee for constructive suggestions. This research made use of NASA's Astrophysics Data System. The Submillimetre Array is a joint project between the Smithsonian Astrophysical Observatory and the Academia Sinica Institute of Astronomy and Astrophysics, and it is funded by the Smithsonian Institution and the Academia Sinica. S.F. acknowledges financial support by the European Community Seventh Framework Programme [FP7/2007-2013] under grant agreement No. 238258. D.S. acknowledges support by the Deutsche Forschungsgemeinschaft through SPP 1385: "The first ten million years of the solar system - a planetary materials approach" (SE 1962/1-2).

\section{References}

Allen, D. A., \& Burton, M. G. 1993, Nature, 363, 54

Andersson, M., Askne, J., \& Hjalmarson, A. 1984, A\&A, 136, 243

Araya, E., Hofner, P., Kurtz, S., Bronfman, L., \& DeDeo, S. 2005, ApJS, 157, 279

Bachiller, R., \& Pérez Gutiérrez, M. 1997, ApJ, 487, L93

Bachiller, R., Pérez Gutiérrez, M., Kumar, M. S. N., \& Tafalla, M. 2001, A\&A, 372,899

Bajaja, E., \& van Albada, G. D. 1979, A\&A, 75, 251

Bally, J., Cunningham, N. J., Moeckel, N., et al. 2011, ApJ, 727, 113

Beckwith, S., Persson, S. E., Neugebauer, G., \& Becklin, E. E. 1978, ApJ, 223, 464

Bell, T. A., Cernicharo, J., Viti, S., et al. 2014, A\&A, 564, A114

Belloche, A., Menten, K. M., Comito, C., et al. 2008, A\&A, 482, 179

Belloche, A., Garrod, R. T., Müller, H. S. P., et al. 2009, A\&A, 499, 215

Bergin, E. A., Snell, R. L., \& Goldsmith, P. F. 1996, ApJ, 460, 343

Bergin, E. A., Phillips, T. G., Comito, C., et al. 2010, A\&A, 521, L20

Beuther, H., \& Nissen, H. D. 2008, ApJ, 679, L121

Beuther, H., Zhang, Q., Greenhill, L. J., et al. 2004, ApJ, 616, L31

Beuther, H., Zhang, Q., Greenhill, L. J., et al. 2005, ApJ, 632, 355

Beuther, H., Zhang, Q., Reid, M. J., et al. 2006, ApJ, 636, 323

Beuther, H., Churchwell, E. B., McKee, C. F., \& Tan, J. C. 2007a, in Protostars and Planets V, eds. B. Reipurth, D. Jewitt \& K. Keil, 165

Beuther, H., Leurini, S., Schilke, P., et al. 2007b, A\&A, 466, 1065

Beuther, H., Linz, H., Bik, A., Goto, M., \& Henning, T. 2010, A\&A, 512, A29

Blake, G. A., Sutton, E. C., Masson, C. R., \& Phillips, T. G. 1987, ApJ, 315, 621

Blake, G. A., Mundy, L. G., Carlstrom, J. E., et al. 1996, ApJ, 472, L49

Boucher, D., Burie, J., Bauer, A., Dubrulle, A., \& Demaison, J. 1980, J. Phys. Chem. Ref. Data, 9, 659

Bronfman, L., Nyman, L.-A., \& May, J. 1996, A\&AS, 115, 81

Brouillet, N., Despois, D., Baudry, A., et al. 2013, A\&A, 550, A46

Caselli, P., Hasegawa, T. I., \& Herbst, E. 1993, ApJ, 408, 548

Chandler, C. J., \& Wood, D. O. S. 1997, MNRAS, 287, 445

Chapman, J. F., Millar, T. J., Wardle, M., Burton, M. G., \& Walsh, A. J. 2009, MNRAS, 394, 221

Charnley, S. B. 2004, Adv. Space Res., 33, 23

Charnley, S. B., Tielens, A. G. G. M., \& Rodgers, S. D. 1997, ApJ, 482, L203

Chen, L., \& Woon, D. E. 2011, J. Phys. Chem. A, 115, 5166

Chernin, L. M., \& Wright, M. C. H. 1996, ApJ, 467, 676

Chernin, L. M., Masson, C. R., \& Fuller, G. A. 1994, ApJ, 436, 741

Chin, Y.-N., Henkel, C., Whiteoak, J. B., Langer, N., \& Churchwell, E. B. 1996, A\&A, 305, 960

Cohen, R. J., Gasiprong, N., Meaburn, J., \& Graham, M. F. 2006, MNRAS, 367, 541

Comito, C., Schilke, P., Phillips, T. G., et al. 2005, ApJS, 156, 127

Crockett, N. R., Bergin, E. A., Neill, J. L., et al. 2014, ApJ, 787, 112

Demyk, K., Wlodarczak, G., \& Carvajal, M. 2008, A\&A, 489, 589

Di Francesco, J., Johnstone, D., Kirk, H., MacKenzie, T., \& Ledwosinska, E. 2008, ApJS, 175, 277

Doeleman, S. S., Lonsdale, C. J., \& Pelkey, S. 1999, ApJ, 510, L55

Doi, T., O’Dell, C. R., \& Hartigan, P. 2002, AJ, 124, 445

Dougados, C., Lena, P., Ridgway, S. T., Christou, J. C., \& Probst, R. G. 1993, ApJ, 406, 112

Downes, D., Genzel, R., Becklin, E. E., \& Wynn-Williams, C. G. 1981, ApJ, 244, 869

Draine, B. T. 2011, Physics of the Interstellar and Intergalactic Medium (Princeton University Press)

Ehrenfreund, P., \& Charnley, S. B. 2000, ARA\&A, 38, 427

Eisner, J. A., Plambeck, R. L., Carpenter, J. M., et al. 2008, ApJ, 683, 304 
S. Feng et al.: Resolving the chemical substructure of Orion-KL

Erickson, N. R., Goldsmith, P. F., Snell, R. L., et al. 1982, ApJ, 261, L103

Esplugues, G. B., Tercero, B., Cernicharo, J., et al. 2013, A\&A, 556, A143

Favre, C., Despois, D., Brouillet, N., et al. 2011a, A\&A, 532, A32

Favre, C., Wootten, H. A., Remijan, A. J., et al. 2011b, ApJ, 739, L12

Fish, V. L., Muehlbrad, T. C., Pratap, P., et al. 2011, ApJ, 729, 14

Friedel, D. N., \& Snyder, L. E. 2008, ApJ, 672, 962

Friedel, D. N., \& Widicus Weaver, S. L. 2011, ApJ, 742, 64

Friedel, D. N., \& Widicus Weaver, S. L. 2012, ApJS, 201, 17

Friedel, D. N., Snyder, L. E., Remijan, A. J., \& Turner, B. E. 2005, ApJ, 632, L95

Garrod, R. T. 2013, ApJ, 765, 60

Garrod, R. T., Weaver, S. L. W., \& Herbst, E. 2008, ApJ, 682, 283

Gaume, R. A., Wilson, T. L., Vrba, F. J., Johnston, K. J., \& Schmid-Burgk, J. 1998, ApJ, 493, 940

Genzel, R., \& Stutzki, J. 1989, ARA\&A, 27, 41

Genzel, R., Reid, M. J., Moran, J. M., \& Downes, D. 1981, ApJ, 244, 884

Gerner, T., Beuther, H., Semenov, D., et al. 2014, A\&A, 563, A97

Goddi, C., Greenhill, L. J., Humphreys, E. M. L., Chandler, C. J., \& Matthews, L. D. 2011a, ApJ, 739, L13

Goddi, C., Humphreys, E. M. L., Greenhill, L. J., Chandler, C. J., \& Matthews, L. D. 2011b, ApJ, 728, 15

Goldsmith, P. F., \& Langer, W. D. 1999, ApJ, 517, 209

Goldsmith, P. F., Snell, R. L., Deguchi, S., Krotkov, R., \& Linke, R. A. 1982 ApJ, 260, 147

Gómez, L., Rodríguez, L. F., Loinard, L., et al. 2005, ApJ, 635, 1166

Gómez, L., Rodríguez, L. F., Loinard, L., et al. 2008, ApJ, 685, 333

Greaves, J. S., \& White, G. J. 1991, A\&AS, 91, 237

Greenhill, L. J., Gwinn, C. R., Schwartz, C., Moran, J. M., \& Diamond, P. J. 1998, Nature, 396, 650

Greenhill, L. J., Gezari, D. Y., Danchi, W. C., et al. 2004, ApJ, 605, L57

Greenhill, L. J., Goddi, C., Chandler, C. J., Matthews, L. D., \& Humphreys, E. M. L. 2013, ApJ, 770, L32

Guélin, M., Brouillet, N., Cernicharo, J., Combes, F., \& Wootten, A. 2008, Ap\&SS, 313, 45

Hasegawa, T. I., \& Herbst, E. 1993, MNRAS, 261, 83

Herbst, E. 1981, Nature, 289, 656

Herbst, E., \& van Dishoeck, E. F. 2009, ARA\&A, 47, 427

Hildebrand, R. H. 1983, QJRAS, 24, 267

Ho, P. T. P., Moran, J. M., \& Lo, K. Y. 2004, ApJ, 616, L1

Hollis, J. M. 1982, ApJ, 260, 159

Hollis, J. M., Lovas, F. J., \& Jewell, P. R. 2000, ApJ, 540, L107

Hollis, J. M., Lovas, F. J., Jewell, P. R., \& Coudert, L. H. 2002, ApJ, 571, L59

Hollis, J. M., Jewell, P. R., Lovas, F. J., \& Remijan, A. 2004, ApJ, 613, L45

Johansson, L. E. B., Andersson, C., Ellder, J., et al. 1984, A\&A, 130, 227

Johnstone, D., \& Bally, J. 1999, ApJ, 510, L49

Kalenskii, S. V., Promislov, V. G., Alakoz, A., Winnberg, A. V., \& Johansson, L. E. B. 2000, A\&A, 354, 1036

Kwan, J., \& Scoville, N. 1976, ApJ, 210, L39

Laas, J. C., Garrod, R. T., Herbst, E., \& Widicus Weaver, S. L. 2011, ApJ, 728, 71

Lacombe, F., Gendron, E., Rouan, D., et al. 2004, A\&A, 417, L5

Lee, J.-K., \& Burton, M. G. 2000, MNRAS, 315, 11

Lee, C. W., \& Cho, S.-H. 2002, J. Kor. Astron. Soc., 35, 187

Lerate, M. R., Yates, J., Viti, S., et al. 2008, MNRAS, 387, 1660

Lis, D. C., Serabyn, E., Keene, J., et al. 1998, ApJ, 509, 299

Liu, S.-Y., Girart, J. M., Remijan, A., \& Snyder, L. E. 2002, ApJ, 576, 255

Loren, R. B., \& Mundy, L. G. 1984, ApJ, 286, 232

Lovas, F. J. 2004, Phys. Chem. Ref., 33, 117

Markwick, A. J., Millar, T. J., \& Charnley, S. B. 2000, ApJ, 535, 256

Martin-Pintado, J., Bachiller, R., \& Fuente, A. 1992, A\&A, 254, 315

Masson, C. R., Claussen, M. J., Lo, K. Y., et al. 1985, ApJ, 295, L47

Masson, C. R., Lo, K. Y., Phillips, T. G., et al. 1987, ApJ, 319, 446

Matthews, L. D., Greenhill, L. J., Goddi, C., et al. 2010, ApJ, 708, 80

McCarthy, M. C., Gottlieb, C. A., Gupta, H., \& Thaddeus, P. 2006, ApJ, 652 L141

Meier, D. S., \& Turner, J. L. 2005, ApJ, 618, 259

Menten, K. M., \& Reid, M. J. 1995, ApJ, 445, L157

Menten, K. M., Reid, M. J., Forbrich, J., \& Brunthaler, A. 2007, A\&A, 474, 515

Miotello, A., Testi, L., Lodato, G., et al. 2014, A\&A, 567, A32

Müller, H. S. P., Schlöder, F., Stutzki, J., \& Winnewisser, G. 2005, J. Mol. Struct., 742,215

Mundy, L. G., Scoville, N. Z., Baath, L. B., Masson, C. R., \& Woody, D. P. 1986, ApJ, 304, L51

Murata, Y., Kawabe, R., Ishiguro, M., et al. 1992, PASJ, 44, 381

Myers, P. C., Linke, R. A., \& Benson, P. J. 1983, ApJ, 264, 517
Neill, J. L., Steber, A. L., Muckle, M. T., et al. 2011, J. Phys. Chem. A, 115, 6472

Niederhofer, F., Humphreys, E. M. L., \& Goddi, C. 2012, A\&A, 548, A69

Nissen, H. D., Gustafsson, M., Lemaire, J. L., et al. 2007, A\&A, 466, 949

Nissen, H. D., Cunningham, N. J., Gustafsson, M., et al. 2012, A\&A, 540, A119

Norris, R. P. 1984, MNRAS, 207, 127

Nummelin, A., Bergman, P., Hjalmarson, A., et al. 2000, ApJS, 128, 213

Olmi, L., Cesaroni, R., \& Walmsley, C. M. 1993, BAAS, 25, 1315

Olofsson, A. O. H., Persson, C. M., Koning, N., et al. 2007, A\&A, 476, 791

Ossenkopf, V., \& Henning, T. 1994, A\&A, 291, 943

Palau, A., Fuente, A., Girart, J. M., et al. 2011, ApJ, 743, L32

Peng, T.-C., Despois, D., Brouillet, N., Parise, B., \& Baudry, A. 2012a, A\&A, 543, A152

Peng, T.-C., Zapata, L. A., Wyrowski, F., Güsten, R., \& Menten, K. M. 2012b, A\&A, 544, L19

Peng, T.-C., Despois, D., Brouillet, N., et al. 2013, A\&A, 554, A78

Persson, C. M., Olofsson, A. O. H., Koning, N., et al. 2007, A\&A, 476, 807

Pickett, H. M., Poynter, R. L., Cohen, E. A., et al. 1998, J. Quant. Spectr. Rad. Transf., 60, 883

Pineau des Forets, G., Roueff, E., Schilke, P., \& Flower, D. R. 1993, MNRAS, 262,915

Plambeck, R. L., Wright, M. C. H., Welch, W. J., et al. 1982, ApJ, 259, 617

Plambeck, R. L., Wright, M. C. H., Friedel, D. N., et al. 2009, ApJ, 704, L25

Plume, R., Bergin, E. A., Phillips, T. G., et al. 2012, ApJ, 744, 28

Purcell, C. R., Balasubramanyam, R., Burton, M. G., et al. 2006, MNRAS, 367, 553

Reid, M. J., Menten, K. M., Greenhill, L. J., \& Chandler, C. J. 2007, ApJ, 664, 950

Remijan, A. J., Leigh, D. P., Markwick-Kemper, A. J., \& Turner, B. E. 2008, ArXiv e-prints [arXiv: 0802 .2273]

Rice, C. A., \& Maier, J. P. 2013, J. Phys. Chem. A, 117, 5559

Rizzo, J. R., Martín-Pintado, J., \& de Vicente, P. 2001, in Highlights of Spanish astrophysics II, eds. J. Zamorano, J. Gorgas, \& J. Gallego, 397

Rodríguez, L. F., Poveda, A., Lizano, S., \& Allen, C. 2005, ApJ, 627, L65

Sanchez-Monge, A. 2011, Ph.D. Thesis, University of Barcelona

Sandstrom, K. M., Peek, J. E. G., Bower, G. C., Bolatto, A. D., \& Plambeck, R. L. 2007, ApJ, 667, 1161

Sault, R. J., Teuben, P. J., \& Wright, M. C. H. 1995, in Astronomical Data Analysis Software and Systems IV, eds. R. A. Shaw, H. E. Payne, \& J. J. E. Hayes, ASP Conf. Ser., 77, 433

Schilke, P., Walmsley, C. M., Pineau des Forets, G., \& Flower, D. R. 1997, A\&A, 321,293

Schilke, P., Benford, D. J., Hunter, T. R., Lis, D. C., \& Phillips, T. G. 2001, ApJS, 132,281

Schreyer, K., \& Henning, T. 1999, in The Orion Complex Revisited, ASP Conf. Ser., 99, 1999

Schuller, F., Menten, K. M., Contreras, Y., et al. 2009, A\&A, 504, 415

Scoville, N. Z., Hall, D. N. B., Ridgway, S. T., \& Kleinmann, S. G. 1982, ApJ, 253, 136

Snyder, L. E., Lovas, F. J., Mehringer, D. M., et al. 2002, ApJ, 578, 245

Snyder, L. E., Lovas, F. J., Hollis, J. M., et al. 2005, ApJ, 619, 914

Sugai, H., Usuda, T., Kataza, H., et al. 1994, ApJ, 420, 746

Sutton, E. C., Blake, G. A., Masson, C. R., \& Phillips, T. G. 1985, ApJS, 58, 341

Sutton, E. C., Peng, R., Danchi, W. C., et al. 1995, ApJS, 97, 455

Tan, J. C., Beltran, M. T., Caselli, P., et al. 2014, in Protostars and Planets VI, eds. H. Beuther, R. S. Klessen, C. P. Dullemond, \& T. Henning (Tucson: University of Arizona Press), 149

Tang, Y.-W., Ho, P. T. P., Koch, P. M., \& Rao, R. 2010, ApJ, 717, 1262

Taylor, K. N. R., Storey, J. W. V., Sandell, G., Williams, P. M., \& Zealey, W. J. 1984, Nature, 311, 236

Tercero, B., Cernicharo, J., Pardo, J. R., \& Goicoechea, J. R. 2010, A\&A, 517, A96

Thorwirth, S. 2001, Ph.D. Thesis, I. Physikalisches Institut Universität zu Köln, Germany

Tideswell, D. M., Fuller, G. A., Millar, T. J., \& Markwick, A. J. 2010, A\&A, 510, A85

Turner, B. E. 1989, ApJS, 70, 539

Turner, B. E. 1991, ApJS, 76, 617

Turner, B. E., Terzieva, R., \& Herbst, E. 1999, ApJ, 518, 699

van der Tak, F. F. S., Black, J. H., Schöier, F. L., Jansen, D. J., \& van Dishoeck, E. F. 2007 , A\&A, 468, 627

van Dishoeck, E. F. 2009, Astrochemistry of Dense Protostellar and Protoplanetary Environments, eds. H. A. Thronson, M. Stiavelli, \& A. Tielens, 187

Vasyunin, A. I., \& Herbst, E. 2013, ApJ, 769, 34 
Viti, S., Codella, C., Benedettini, M., \& Bachiller, R. 2004, MNRAS, 350, 1029

Wakelam, V., Caselli, P., Ceccarelli, C., Herbst, E., \& Castets, A. 2004, A\&A, 422, 159

Wang, S., Bergin, E. A., Crockett, N. R., et al. 2011, A\&A, 527, A95

Widicus Weaver, S. L., \& Friedel, D. N. 2012, ApJS, 201, 16

Wilner, D. J., Wright, M. C. H., \& Plambeck, R. L. 1994, ApJ, 422, 642

Wilson, T. L., \& Rood, R. 1994, ARA\&A, 32, 191

Wilson, T. L., Gaume, R. A., Gensheimer, P., \& Johnston, K. J. 2000, ApJ, 538 665

Wilson, T. L., Rohlfs, K., \& Hüttemeister, S. 2009, Tools of Radio Astronomy (Springer-Verlag)

Wilson, T. L., Muders, D., Dumke, M., Henkel, C., \& Kawamura, J. H. 2011, ApJ, 728, 61

Woody, D. P., Scott, S. L., Scoville, N. Z., et al. 1989, ApJ, 337, L41

Wright, M. C. H., \& Plambeck, R. L. 1983, ApJ, 267, L115
Wright, M., Sandell, G., Wilner, D. J., \& Plambeck, R. L. 1992, ApJ, 393, 225 Wright, M. C. H., Plambeck, R. L., \& Wilner, D. J. 1995, in NCSA Astronomy Data Image Library, 2

Wright, M. C. H., Plambeck, R. L., \& Wilner, D. J. 1996, ApJ, 469, 216

Wynn-Williams, C. G., Genzel, R., Becklin, E. E., \& Downes, D. 1984, ApJ, 281,172

Zapata, L. A., Schmid-Burgk, J., Ho, P. T. P., Rodríguez, L. F., \& Menten, K. M. 2009, ApJ, 704, L45

Zapata, L. A., Schmid-Burgk, J., \& Menten, K. M. 2011, A\&A, 529, A24

Zapata, L. A., Rodríguez, L. F., Schmid-Burgk, J., et al. 2012, ApJ, 754, L17

Zeng, Q., Mao, P. Q., \& Pei, C. C. 2006, Microwave spectrum of astrophysics diagnosis (Chinese edition)

Zinchenko, I., Henkel, C., \& Mao, R. Q. 2000, A\&A, 361, 1079

Zinnecker, H., \& Yorke, H. W. 2007, ARA\&A, 45, 481

Ziurys, L. M., \& McGonagle, D. 1993, ApJS, 89, 155

Zuckerman, B., Kuiper, T. B. H., \& Rodriguez Kuiper, E. N. 1976, ApJ, 209, L137 


\section{Appendix A}

Table A.1. Identified emission lines from the SMA-30 m combined dataset.

\begin{tabular}{|c|c|c|c|c|c|c|}
\hline $\begin{array}{l}\text { Freq. } \\
\text { (GHz) }\end{array}$ & Mol. & $\begin{array}{r}E_{\mathrm{u}} / k_{\mathrm{B}} \\
(\mathrm{K}) \\
\end{array}$ & & $\begin{array}{l}\text { Freq. } \\
(\mathrm{GHz})\end{array}$ & Mol. (candidates) & $\begin{array}{r}E_{\mathrm{u}} / k_{\mathrm{B}} \\
(\mathrm{K}) \\
\end{array}$ \\
\hline 218.903 & $\operatorname{OCS}_{(v=0)}(18 \rightarrow 17)$ & 100 & & & & \\
\hline 218.966 & $\mathrm{HCOOCH}_{3(v=1)}\left(18_{12,6} \rightarrow 17_{12,5}\right) \mathrm{E}$ & 384 & & & & \\
\hline 218.981 & $\operatorname{HNCO}_{(v=0)}\left(10_{1,10} \rightarrow 9_{1,9}\right)$ & 101 & & & & \\
\hline 219.068 & $\mathrm{HCOOCH}_{3(v=1)}\left(18_{17,2} \rightarrow 17_{17,1}\right) \mathrm{E}$ & 481 & & & & \\
\hline 219.079 & $\mathrm{HCOOCH}_{3(v=1)}\left(28_{3,25} \rightarrow 28_{2,26}\right) \mathrm{E}$ & 434 & & & & \\
\hline 219.090 & $\mathrm{HCOOCH}_{3(v=0)}\left(34_{7,28} \rightarrow 34_{5,29}\right) \mathrm{E}$ & 387 & $*$ & 219.090 & $\mathrm{HCOOCH}_{3(v=0)}\left(34_{7,28} \rightarrow 34_{5,29}\right) \mathrm{A}$ & 387 \\
\hline 219.154 & $\mathrm{HCOOCH}_{3(v=1)}\left(18_{11,7} \rightarrow 17_{11,6}\right) \mathrm{E}$ & 369 & $*$ & 219.153 & $\mathrm{HCOOCH}_{3(v=1)}\left(10_{4,6} \rightarrow 9_{3,6}\right) \mathrm{E}$ & 230 \\
\hline 219.174 & $\mathrm{HC}_{3} \mathrm{~N}_{\left(v_{7}=1\right)}(24 \rightarrow 23)$ & 452 & $\dagger$ & & & \\
\hline 219.195 & $\mathrm{HCOOCH}_{3(v=1)}\left(18_{16,3} \rightarrow 17_{16,2}\right) \mathrm{E}$ & 459 & $*$ & 219.196 & $\mathrm{HCOOCH}_{3(v=0)}\left(36_{6,30} \rightarrow 36_{6,31}\right) \mathrm{E}$ & 429 \\
\hline \multirow[t]{3}{*}{219.220} & $\mathrm{CH}_{3} \mathrm{COCH}_{3(v=0)}\left(21_{1,20} \rightarrow 20_{2,19}\right) \mathrm{AE}$ & 122 & $*$ & 219.220 & $\mathrm{CH}_{3} \mathrm{COCH}_{3(v=0)}\left(21_{2,20} \rightarrow 20_{1,19}\right) \mathrm{AE}$ & 122 \\
\hline & & & & 219.220 & $\mathrm{CH}_{3} \mathrm{COCH}_{3(v=0)}\left(21_{2,20} \rightarrow 20_{2,19}\right) \mathrm{EA}$ & 122 \\
\hline & & & & 219.220 & $\mathrm{CH}_{3} \mathrm{COCH}_{3(v=0)}\left(21_{1,20} \rightarrow 20_{1,19}\right) \mathrm{EA}$ & 122 \\
\hline \multirow[t]{3}{*}{219.242} & $\mathrm{CH}_{3} \mathrm{COCH}_{3(v=0)}\left(21_{1,20} \rightarrow 20_{2,19}\right) \mathrm{EE}$ & 122 & * & 219.242 & $\mathrm{CH}_{3} \mathrm{COCH}_{3(v=0)}\left(21_{2,20} \rightarrow 20_{1,19}\right) \mathrm{EE}$ & 122 \\
\hline & & & & 219.242 & $\mathrm{CH}_{3} \mathrm{COCH}_{3(v=0)}\left(21_{2,20} \rightarrow 20_{2,19}\right) \mathrm{EE}$ & 122 \\
\hline & & & & 219.220 & $\mathrm{CH}_{3} \mathrm{COCH}_{3(v=0)}\left(21_{1,20} \rightarrow 20_{1,19}\right) \mathrm{EE}$ & 122 \\
\hline 219.264 & $\mathrm{CH}_{3} \mathrm{COCH}_{3(v=0)}\left(21_{1,20} \rightarrow 20_{2,19}\right) \mathrm{AA}$ & 122 & * & 219.264 & $\mathrm{CH}_{3} \mathrm{COCH}_{3(v=0)}\left(21_{2,20} \rightarrow 20_{1,19}\right) \mathrm{AA}$ & 122 \\
\hline 219.276 & $\mathrm{SO}_{2(v=0)}\left(22_{7,15} \rightarrow 23_{6,18}\right)$ & 352 & & & & \\
\hline 219.311 & $\mathrm{CH}_{3} \mathrm{COCH}_{3(v=0)}\left(12_{9,4} \rightarrow 11_{8,3}\right) \mathrm{AA}$ & 66 & & & & \\
\hline 219.325 & UL & & $?$ & & & \\
\hline 219.331 & $\mathrm{HCOOCH}_{3(v=1)}\left(18_{15,4} \rightarrow 17_{15,3)}\right) \mathrm{E}$ & 438 & $*$ & 219.328 & $\mathrm{HCOOCH}_{3(v=1)}\left(36_{9,28} \rightarrow 36_{8,29}\right) \mathrm{E}$ & 635 \\
\hline 219.355 & ${ }^{34} \mathrm{SO}_{2(v=0)}\left(11_{1,11} \rightarrow 10_{0,10}\right)$ & 60 & $\dagger$ & & & \\
\hline 219.401 & $\mathrm{CH}_{2} \mathrm{CHCN}_{(v=0)}\left(23_{3,20} \rightarrow 22_{3,19}\right)$ & 145 & & & & \\
\hline 219.412 & $\mathrm{HCOOCH}_{3(v=1)}\left(18_{10,8} \rightarrow 17_{10,7}\right) \mathrm{E}$ & 355 & & & & \\
\hline 219.417 & $\mathrm{HCOOCH}_{3(v=0)}\left(30_{5,26} \rightarrow 30_{4,27}\right) \mathrm{E}$ & 291 & & & & \\
\hline 219.463 & $\mathrm{CH}_{3} \mathrm{CH}_{2} \mathrm{CN}\left(22_{2,21} \rightarrow 21_{1,20}\right)$ & 112 & & & & \\
\hline 219.479 & $\mathrm{HCOOCH}_{3(v=1)}\left(18_{14,5} \rightarrow 17_{14,4}\right) \mathrm{E}$ & 419 & & & & \\
\hline 219.484 & $\mathrm{HCOOCH}_{3(v=0)}\left(30_{5,26} \rightarrow 30_{4,27}\right) \mathrm{A}$ & 291 & & & & \\
\hline 219.506 & $\mathrm{CH}_{3} \mathrm{CH}_{2} \mathrm{CN}_{(v=0)}\left(24_{2,22} \rightarrow 23_{2,21}\right)$ & 136 & & & & \\
\hline 219.547 & $\operatorname{HNCO}_{(v=0)}\left(10_{4,6} \rightarrow 9_{4,5}\right)$ & 750 & $*$ & 219.547 & $\mathrm{HNCO}_{(v=0)}\left(10_{4,7} \rightarrow 9_{4,6}\right)$ & 750 \\
\hline 219.560 & $\mathrm{C}^{18} \mathrm{O}(2 \rightarrow 1)$ & 16 & $\dagger$ & & & \\
\hline 219.566 & $\mathrm{HCOOCH}_{3(v=1)}\left(18_{15,4} \rightarrow 17_{15,3}\right) \mathrm{A}$ & 438 & * & 219.566 & $\mathrm{HCOOCH}_{3(v=1)}\left(18_{15,3} \rightarrow 17_{15,2}\right) \mathrm{A}$ & 438 \\
\hline 219.568 & $\mathrm{HCOOCH}_{3(v=1)}\left(18_{14,5} \rightarrow 17_{14,4}\right) \mathrm{A}$ & 419 & $*$ & 219.568 & $\mathrm{HCOOCH}_{3(v=1)}\left(18_{14,4} \rightarrow 17_{14,3}\right) \mathrm{A}$ & 419 \\
\hline 219.571 & $\mathrm{HCOOCH}_{3(v=1)}\left(18_{16,3} \rightarrow 17_{16,2}\right) \mathrm{A}$ & 458 & * & 219.571 & $\mathrm{HCOOCH}_{3(v=1)}\left(18_{16,2} \rightarrow 17_{16,1}\right) \mathrm{A}$ & 458 \\
\hline \multirow[t]{3}{*}{219.579} & $\mathrm{HCOOCH}_{3(v=0)}\left(28_{9,19} \rightarrow 28_{8,20}\right) \mathrm{A}$ & 294 & * & 219.579 & $\mathrm{HCOOCH}_{3(v=1)}\left(18_{17,2} \rightarrow 17_{17,1}\right) \mathrm{A}$ & 481 \\
\hline & & & & 219.579 & $\mathrm{HCOOCH}_{3(v=1)}\left(18_{17,1} \rightarrow 17_{17,0}\right) \mathrm{A}$ & 481 \\
\hline & & & & 219.578 & $\mathrm{HCOOCH}_{3(v=1)}\left(29_{9,21} \rightarrow 29_{8,21}\right) \mathrm{E}$ & 498 \\
\hline \multirow[t]{2}{*}{219.584} & $\mathrm{HCOOCH}_{3(v=1)}\left(18_{13,6} \rightarrow 17_{13,5}\right) \mathrm{A}$ & 401 & $*$ & 219.584 & $\mathrm{HCOOCH}_{3(v=1)}\left(18_{13,5} \rightarrow 17_{13,4}\right) \mathrm{A}$ & 401 \\
\hline & & & & 219.586 & $\mathrm{HCOOCH}_{3(v=0}\left(30_{9,22} \rightarrow 30_{8,23}\right) \mathrm{A}$ & 330 \\
\hline 219.592 & $\mathrm{HCOOCH}_{3(v=0}\left(28_{9,19} \rightarrow 28_{8,20}\right) \mathrm{E}$ & 294 & & & & \\
\hline 219.600 & $\mathrm{HCOOCH}_{3(v=0}\left(30_{9,22} \rightarrow 30_{8,23}\right) \mathrm{E}$ & 330 & & & & \\
\hline \multirow[t]{2}{*}{219.607} & $\mathrm{HCOOCH}_{3(v=0}\left(30_{5,26} \rightarrow 30_{3,27}\right) \mathrm{E}$ & 291 & * & 219.610 & $\mathrm{CH}_{3} \mathrm{COCH}_{3(v=0)}\left(33_{8,25} \rightarrow 33_{8,26}\right) \mathrm{EE}$ & 368 \\
\hline & & & & 219.610 & $\mathrm{CH}_{3} \mathrm{COCH}_{3(v=0)}\left(33_{9,25} \rightarrow 33_{7,26}\right) \mathrm{EE}$ & 368 \\
\hline 219.622 & $\mathrm{HCOOCH}_{3(v=1)}\left(18_{12,7} \rightarrow 17_{12,6}\right) \mathrm{A}$ & 384 & $*$ & 219.622 & $\mathrm{HCOOCH}_{3(v=1)}\left(18_{12,6} \rightarrow 17_{12,5}\right) \mathrm{A}$ & 384 \\
\hline 219.642 & $\mathrm{HCOOCH}_{3(v=1)}\left(18_{13,6} \rightarrow 17_{13,5}\right) \mathrm{E}$ & 401 & & & & \\
\hline 219.657 & $\operatorname{HNCO}\left(10_{3,8} \rightarrow 9_{3,7}\right)$ & 447 & $*$ & 219.657 & $\operatorname{HNCO}\left(10_{3,7} \rightarrow 9_{3,6}\right)$ & 447 \\
\hline 219.696 & $\mathrm{HCOOCH}_{3(v=1)}\left(18_{11,8} \rightarrow 17_{11,7}\right) \mathrm{A}$ & 369 & $*$ & 219.696 & $\mathrm{HCOOCH}_{3(v=1)}\left(18_{11,7} \rightarrow 17_{11,6}\right) \mathrm{A}$ & 369 \\
\hline 219.705 & $\mathrm{HCOOCH}_{3(v=1)}\left(18_{4,15} \rightarrow 17_{4,14}\right) \mathrm{A}$ & 299 & & & & \\
\hline 219.734 & $\operatorname{HNCO}_{(v=0)}\left(10_{2,9} \rightarrow 9_{2,8}\right)$ & 231 & & & & \\
\hline 219.737 & $\mathrm{HNCO}_{(v=0)}\left(10_{2,8} \rightarrow 9_{2,7}\right)$ & 231 & & & & \\
\hline 219.764 & $\mathrm{HCOOCH}_{3(v=1)}\left(18_{9,9} \rightarrow 17_{9,8}\right) \mathrm{E}$ & 342 & & & & \\
\hline 219.798 & $\operatorname{HNCO}_{(v=0)}\left(10_{0,10} \rightarrow 9_{0,9}\right)$ & 58 & $\dagger$ & & & \\
\hline 219.822 & $\mathrm{HCOOCH}_{3(v=1)}\left(18_{10,9} \rightarrow 17_{10,8}\right) \mathrm{A}$ & 355 & $*$ & 219.822 & $\mathrm{HCOOCH}_{3(v=1)}\left(18_{10,8} \rightarrow 17_{10,7}\right) \mathrm{A}$ & 355 \\
\hline 219.827 & $\mathrm{HCOOCH}_{3(v=1)}\left(18_{12,7} \rightarrow 17_{12,6}\right) \mathrm{E}$ & 384 & & & & \\
\hline
\end{tabular}

Notes. 1) Due to the frequency resolution $\left(0.8125 \mathrm{MHz}, \sim 1.2 \mathrm{~km} \mathrm{~s}^{-1}\right)$, lines with broad FWHM width can be attributed to different species, so lines with stronger CDMS/JPL intensity are listed here in the left column with "**, and the potential blended weaker transitions are listed in the right column. 2) Tentative detections and unidentified lines are marked with "?". 3) Lines with " $\dagger$ ” are imaged in Fig. 4, with “t” are imaged in Fig. 5. 4) "A(E)A(E)" represents 4 types of transitions are possible: AA, EE, AE, EA. 
Table A.1. continued.

\begin{tabular}{|c|c|c|c|c|c|c|}
\hline \multirow{2}{*}{$\begin{array}{l}\text { Freq. } \\
(\mathrm{GHz}) \\
219.909\end{array}$} & Mol. & \multicolumn{2}{|c|}{$\begin{array}{r}E_{\mathrm{u}} / k_{\mathrm{B}} \\
(\mathrm{K})\end{array}$} & \multirow[t]{2}{*}{$\begin{array}{l}\text { Freq. } \\
(\mathrm{GHz})\end{array}$} & \multicolumn{2}{|c|}{$\begin{array}{r}\text { Mol. (candidates) } E_{\mathrm{u}} / k_{\mathrm{B}} \\
(\mathrm{K})\end{array}$} \\
\hline & $\mathrm{H}_{2}^{13} \mathrm{CO}\left(3_{1,2} \rightarrow 2_{1,1}\right)$ & 33 & $\dagger$ & & & \\
\hline 219.949 & $\mathrm{SO}_{(v=0)}\left(6_{5} \rightarrow 5_{4}\right)$ & 35 & $\dagger$ & & & \\
\hline 219.984 & $\mathrm{CH}_{3} \mathrm{OH}_{\left(v_{\mathrm{t}}=0\right)}\left(25_{3,22} \rightarrow 24_{4,20}\right) \mathrm{E}$ & 802 & & & & \\
\hline 219.994 & $\mathrm{CH}_{3} \mathrm{OH}_{\left(v_{\mathrm{t}}=0\right)}\left(23_{5,19} \rightarrow 22_{6,17}\right) \mathrm{E}$ & 776 & & & & \\
\hline 220.030 & $\mathrm{HCOOCH}_{3(v=1)}\left(18_{9,10} \rightarrow 17_{9,9}\right) \mathrm{A}$ & 342 & $*$ & 220.030 & $\mathrm{HCOOCH}_{3(v=1)}\left(18_{9,9} \rightarrow 17_{9,8}\right) \mathrm{A}$ & 342 \\
\hline 220.043 & $\mathrm{HCOOCH}_{3(v=1)}\left(18_{11,8} \rightarrow 17_{11,7}\right) \mathrm{E}$ & 368 & & & & \\
\hline 220.078 & $\mathrm{CH}_{3} \mathrm{OH}_{\left(v_{\mathrm{t}}=0\right)}\left(8_{0,8} \rightarrow 7_{1,6}\right) \mathrm{E}$ & 97 & & & & \\
\hline 220.154 & $\mathrm{t}-\mathrm{CH}_{3} \mathrm{CH}_{2} \mathrm{OH}\left(24_{3,22} \rightarrow 24_{2,23}\right)$ & 263 & & & & \\
\hline 220.167 & $\mathrm{HCOOCH}_{3(v=0)}\left(17_{4,13} \rightarrow 16_{4,12}\right) \mathrm{E}$ & 103 & $\ddagger$ & & & \\
\hline 220.178 & $\mathrm{CH}_{2} \mathrm{CO}\left(11_{1,11} \rightarrow 10_{1,10}\right)$ & 76 & $\dagger$ & & & \\
\hline 220.190 & $\mathrm{HCOOCH}_{3(v=0)}\left(17_{4,13} \rightarrow 16_{4,12}\right) \mathrm{A}$ & 103 & & & & \\
\hline 220.258 & $\mathrm{HCOOCH}_{3(v=1)}\left(18_{8,10} \rightarrow 17_{8,9}\right) \mathrm{E}$ & 330 & $\ddagger$ & & & \\
\hline 220.296 & $\mathrm{CH}_{3}^{13} \mathrm{CN}\left(12_{9} \rightarrow 11_{9}\right)$ & 646 & & & & \\
\hline 220.307 & $\mathrm{HCOOCH}_{3(v=1)}\left(18_{10,9} \rightarrow 17_{10,8}\right) \mathrm{E}$ & 354 & & & & \\
\hline 220.324 & $\mathrm{CH}_{3} \mathrm{CN}_{(v=0)}\left(12_{10} \rightarrow 11_{10}\right)$ & 782 & & & & \\
\hline 220.355 & $\mathrm{CH}_{3} \mathrm{COCH}_{3(v=0)}\left(22_{0,22} \rightarrow 21_{0,21}\right)$ & 124 & $\mathrm{AE}, \mathrm{EA}^{*}$ & 220.355 & $\mathrm{CH}_{3} \mathrm{COCH}_{3(v=0)}\left(22_{1,22} \rightarrow 21_{1,21}\right)$ & $124 \mathrm{AE}, \mathrm{EA}$ \\
\hline & & & & 220.355 & $\mathrm{C}_{6} \mathrm{H}(\mathrm{J}=159 / 2-157 / 2, \Omega=3 / 2, \mathrm{l}=\mathrm{e})$ & $425 ?$ \\
\hline 220.362 & $\mathrm{CH}_{3} \mathrm{COCH}_{3(v=0)}\left(22_{0,22} \rightarrow 21_{1,21}\right) \mathrm{EE}$ & 124 & $*$ & 220.362 & $\mathrm{CH}_{3} \mathrm{COCH}_{3(v=0)}\left(22_{1,22} \rightarrow 21_{1,21}\right) \mathrm{EE}$ & 124 \\
\hline & & & & 220.362 & $\mathrm{CH}_{3} \mathrm{COCH}_{3(v=0)}\left(22_{1,22} \rightarrow 21_{0,21}\right) \mathrm{EE}$ & 124 \\
\hline & & & & 220.362 & $\mathrm{CH}_{3} \mathrm{COCH}_{3(v=0)}\left(22_{0,22} \rightarrow 21_{0,21}\right) \mathrm{EE}$ & 124 \\
\hline 220.368 & $\mathrm{HCOOCH}_{3(v=1)}\left(18_{8,11} \rightarrow 17_{8,10}\right) \mathrm{A}$ & 331 & $*$ & 220.370 & $\mathrm{HCOOCH}_{3(v=1)}\left(18_{8,10} \rightarrow 17_{8,9}\right) \mathrm{A}$ & 331 \\
\hline & & & & 220.368 & $\mathrm{CH}_{3} \mathrm{COCH}_{3(v=0)}\left(22_{1,22} \rightarrow 21_{1,21}\right) \mathrm{AA}$ & 124 \\
\hline & & & & 220.368 & $\mathrm{CH}_{3} \mathrm{COCH}_{3(v=0)}\left(22_{0,22} \rightarrow 21_{0,21}\right) \mathrm{AA}$ & 124 \\
\hline & & & & 220.368 & $\mathrm{CH}_{3}^{13} \mathrm{CN}\left(12_{8} \rightarrow 11_{8}\right)$ & 526 \\
\hline 220.399 & ${ }^{13} \mathrm{CO}(2 \rightarrow 1)$ & 16 & $\dagger$ & & & \\
\hline 220.404 & $\mathrm{CH}_{3} \mathrm{CN}_{(v=0)}\left(12_{9} \rightarrow 11_{9}\right)$ & 646 & & & & \\
\hline 220.416 & $\mathrm{HCOOCH}_{3(v=1)}\left(18_{3,16} \rightarrow 17_{2,15}\right) \mathrm{A}$ & 293 & & & & \\
\hline 220.431 & $\mathrm{CH}_{3}^{13} \mathrm{CN}\left(12_{7} \rightarrow 11_{7}\right)$ & 417 & * & 220.433 & $\mathrm{HCOOCH}_{3(v=0)}\left(35_{5,28} \rightarrow 33_{5,29}\right) \mathrm{A}$ & 357 \\
\hline 220.446 & $\mathrm{CH}_{3} \mathrm{CHO}\left(13_{3,10} \rightarrow 13_{2,11} v=0\right) \mathrm{E}$ & 105 & $?^{*}$ & & & \\
\hline 220.465 & $\mathrm{CH}_{3} \mathrm{COCH}_{3(v=0)}\left(11_{11,1} \rightarrow 10_{10,0}\right) \mathrm{AE}$ & 63 & $*$ & 220.466 & $\mathrm{CH}_{3} \mathrm{COCH}_{3(v=0)}\left(11_{11,0} \rightarrow 10_{10,1}\right) \mathrm{AE}$ & 63 \\
\hline 220.476 & $\mathrm{CH}_{3} \mathrm{CN}_{(v=0)}\left(12_{8} \rightarrow 11_{8}\right)$ & 526 & & & & \\
\hline 220.486 & $\mathrm{CH}_{3}^{13} \mathrm{CN}\left(12_{6} \rightarrow 11_{6}\right)$ & 326 & $*$ & 220.487 & $\mathrm{C}_{6} \mathrm{H}(\mathrm{J}=159 / 2-157 / 2, \Omega=3 / 2, \mathrm{l}=\mathrm{f})$ & 426 \\
\hline 220.525 & $\mathrm{HCOOCH}_{3(v=1)}\left(10_{4,6} \rightarrow 9_{3,7}\right) \mathrm{A}$ & 231 & & & & \\
\hline 220.533 & $\mathrm{CH}_{3}^{13} \mathrm{CN}\left(12_{5} \rightarrow 11_{5}\right)$ & 247 & & & & \\
\hline 220.539 & $\mathrm{CH}_{3} \mathrm{CN}_{(v=0)}\left(12_{7} \rightarrow 11_{7}\right)$ & 419 & & & & \\
\hline 220.561 & $\mathrm{CH}_{2} \mathrm{CHCN}\left(24_{1,24} \rightarrow 23_{1,23}\right)$ & 134 & & & & \\
\hline 220.570 & $\mathrm{CH}_{3}^{13} \mathrm{CN}\left(12_{4} \rightarrow 11_{4}\right)$ & 183 & & & & \\
\hline 220.585 & $\operatorname{HNCO}_{(v=0)}^{3}\left(10_{1,9} \rightarrow 9_{1,8}\right)$ & 101 & & & & \\
\hline 220.594 & $\mathrm{CH}_{3} \mathrm{CN}_{(v=0)}\left(12_{6} \rightarrow 11_{6}\right)$ & 326 & & & & \\
\hline 220.621 & $\mathrm{CH}_{3}^{13} \mathrm{CN}\left(12_{2} \rightarrow 11_{2}\right)$ & 97 & $\ddagger$ & & & \\
\hline 220.641 & $\mathrm{CH}_{3} \mathrm{CN}_{(v=0)}\left(12_{5} \rightarrow 11_{5}\right)$ & 248 & & & & \\
\hline 220.661 & $\mathrm{CH}_{3} \mathrm{CH}_{2} \mathrm{CN}_{(v=0)}\left(25_{2,24} \rightarrow 24_{2,23}\right)$ & 143 & & & & \\
\hline 220.679 & $\mathrm{CH}_{3} \mathrm{CN}_{(v=0)}\left(12_{4} \rightarrow 11_{4}\right)$ & 183 & & & & \\
\hline 220.709 & $\mathrm{CH}_{3} \mathrm{CN}_{(v=0)}\left(12_{3} \rightarrow 11_{3}\right)$ & 133 & & & & \\
\hline 220.730 & $\mathrm{CH}_{3} \mathrm{CN}_{(v=0)}\left(12_{2} \rightarrow 11_{2}\right)$ & 98 & $\ddagger$ & & & \\
\hline 220.743 & $\mathrm{CH}_{3} \mathrm{CN}_{(v=0)}\left(12_{1} \rightarrow 11_{1}\right)$ & 76 & & & & \\
\hline 220.747 & $\mathrm{CH}_{3} \mathrm{CN}_{(v=0)}\left(12_{0} \rightarrow 11_{0}\right)$ & 69 & & & & \\
\hline 220.764 & $\mathrm{CH}_{3} \mathrm{COCH}_{3(v=0)}\left(11_{11,0} \rightarrow 10_{10,0}\right) \mathrm{EE}$ & 63 & & & & \\
\hline 220.786 & $\mathrm{HCOOCH}_{3(v=0)}\left(28_{3,25} \rightarrow 28_{3,26}\right) \mathrm{E}$ & 248 & & & & \\
\hline 220.812 & $\mathrm{HCOOCH}_{3(v=0)}\left(18_{3,16} \rightarrow 17_{2,15}\right) \mathrm{E}$ & 105 & & & & \\
\hline 220.815 & $\mathrm{HCOOCH}_{3(v=0)}\left(18_{3,16} \rightarrow 17_{2,15}\right) \mathrm{A}$ & 105 & & & & \\
\hline 220.820 & $\mathrm{HCOOCH}_{3(v=0)}\left(24_{2,23} \rightarrow 24_{1,24}\right) \mathrm{A}$ & 169 & & & & \\
\hline 220.848 & $\mathrm{CH}_{3} \mathrm{OCH}_{3}\left(24_{4,20} \rightarrow 23_{5,19}\right)$ & 297 & $\mathrm{~A}(\mathrm{E}) \mathrm{A}(\mathrm{E})$ & & & \\
\hline 220.866 & $\mathrm{HCOOCH}_{3(v=0)}\left(28_{3,25} \rightarrow 28_{3,26}\right) \mathrm{A}$ & 248 & $*$ & 220.866 & $\mathrm{HCOOCH}_{3(v=1)}\left(19_{2,17} \rightarrow 18_{3,16}\right) \mathrm{A}$ & 303 \\
\hline 220.889 & $\mathrm{HCOOCH}_{3(v=0)}\left(18_{17,1} \rightarrow 17_{17,0}\right) \mathrm{A}$ & 293 & $*$ & 220.889 & $\mathrm{HCOOCH}_{3(v=0)}\left(18_{17,2} \rightarrow 17_{17,1}\right) \mathrm{A}$ & 293 \\
\hline 220.893 & $\mathrm{CH}_{3} \mathrm{OCH}_{3}\left(23_{4,20} \rightarrow 23_{3,21}\right)$ & 274 & $A(E) A(E)$ & & & \\
\hline 220.901 & $\mathrm{HCOOCH}_{3(v=0)}\left(18_{17,1} \rightarrow 17_{17,0}\right) \mathrm{E}$ & 293 & & & & \\
\hline 220.913 & $\mathrm{HCOOCH}_{3(v=1)}\left(18_{7,12} \rightarrow 17_{7,11}\right) \mathrm{A}$ & 320 & $*$ & 220.910 & $\mathrm{HCOOCH}_{3(v=0)}\left(18_{17,2} \rightarrow 17_{17,1}\right) \mathrm{E}$ & 293 \\
\hline
\end{tabular}


Table A.1. continued.

\begin{tabular}{|c|c|c|c|c|c|c|}
\hline $\begin{array}{l}\text { Freq. } \\
(\mathrm{GHz})\end{array}$ & Mol. & $\begin{array}{r}E_{\mathrm{u}} / k_{\mathrm{B}} \\
(\mathrm{K})\end{array}$ & & $\begin{array}{l}\text { Freq. } \\
(\mathrm{GHz})\end{array}$ & Mol. (candidates) & $\begin{array}{r}E_{\mathrm{u}} / k_{\mathrm{B}} \\
(\mathrm{K}) \\
\end{array}$ \\
\hline 220.926 & $\mathrm{HCOOCH}_{3(v=0)}\left(18_{16,2} \rightarrow 17_{16,1}\right) \mathrm{A}$ & 271 & * & 220.926 & $\mathrm{HCOOCH}_{3(v=0)}\left(18_{16,3} \rightarrow 17_{16,2}\right) \mathrm{A}$ & 271 \\
\hline 220.935 & $\mathrm{HCOOCH}_{3(v=0)}\left(18_{16,2} \rightarrow 17_{16,1}\right) \mathrm{E}$ & 271 & & & & \\
\hline 220.946 & $\mathrm{HCOOCH}_{3(v=1)}\left(18_{7,11} \rightarrow 17_{7,10}\right) \mathrm{A}$ & 321 & $*$ & 220.947 & $\mathrm{HCOOCH}_{3(v=0)}\left(18_{16,3} \rightarrow 17_{16,2}\right) \mathrm{E}$ & 271 \\
\hline 220.967 & $\mathrm{HC}_{7} \mathrm{~N}_{(v=0)}(\mathrm{J}=196-195)$ & 1045 & $?$ & & & \\
\hline 220.978 & $\mathrm{HCOOCH}_{3(v=0)}\left(18_{15,4} \rightarrow 17_{15,3}\right) \mathrm{A}$ & 250 & $*$ & 220.978 & $\mathrm{HCOOCH}_{3(v=0)}\left(18_{15,3} \rightarrow 17_{15,2}\right) \mathrm{A}$ & 250 \\
\hline 220.985 & $\mathrm{HCOOCH}_{3(v=1)}\left(18_{7,11} \rightarrow 17_{7,10}\right) \mathrm{E}$ & 320 & $*$ & 220.984 & $\mathrm{HCOOCH}_{3(v=0)}\left(18_{15,3} \rightarrow 17_{15,2}\right) \mathrm{E}$ & 250 \\
\hline 220.998 & $\mathrm{HCOOCH}_{3(v=0)}\left(18_{15,4} \rightarrow 17_{15,3}\right) \mathrm{E}$ & 250 & $*$ & 220.999 & $\mathrm{~g}-\mathrm{CH}_{3} \mathrm{CH}_{2} \mathrm{OH}\left(13_{0,13} \rightarrow 12_{0,12} v_{\mathrm{t}}=0-1\right)$ & 136 \\
\hline \multirow[t]{2}{*}{221.048} & $\mathrm{HCOOCH}_{3(v=0)}\left(18_{14,5} \rightarrow 17_{14,4}\right) \mathrm{A}$ & 231 & $*$ & 221.048 & $\mathrm{HCOOCH}_{3(v=0)}\left(18_{14,4} \rightarrow 1_{14,3}\right) \mathrm{A}$ & 231 \\
\hline & & & & 221.048 & $\mathrm{HCOOCH}_{3(v=0)}\left(18_{14,4} \rightarrow 17_{14,3}\right) \mathrm{E}$ & 231 \\
\hline 221.067 & $\mathrm{HCOOCH}_{3(v=0)}\left(18_{14,5} \rightarrow 17_{14,4}\right) \mathrm{A}$ & 231 & & & & \\
\hline 221.076 & $\mathrm{HCOOCH}_{3(v=0)}\left(29_{9,21} \rightarrow 29_{8,22}\right) \mathrm{A}$ & 312 & & & & \\
\hline 221.086 & $\mathrm{HCOOCH}_{3(v=0)}\left(29_{9,21} \rightarrow 29_{8,22}\right) \mathrm{E}$ & 312 & & & & \\
\hline 221.111 & $\mathrm{HCOOCH}_{3(v=1)}\left(18_{8,11} \rightarrow 17_{8,10}\right) \mathrm{E}$ & 330 & & & & \\
\hline 221.115 & ${ }^{34} \mathrm{SO}_{2(v=0)}\left(22_{2,20} \rightarrow 22_{1,21}\right)$ & 248 & & & & \\
\hline 221.124 & $\mathrm{CH}_{2} \mathrm{CHCN}_{(v=0)}\left(23_{1,22} \rightarrow 22_{1,21}\right)$ & 130 & & & & \\
\hline \multirow[t]{2}{*}{221.140} & $\mathrm{HCOOCH}_{3(v=0)}\left(18_{13,5} \rightarrow 17_{13,4}\right) \mathrm{E}$ & 213 & $*$ & 221.141 & $\mathrm{HCOOCH}_{3(v=0)}\left(18_{13,5} \rightarrow 17_{13,4}\right) \mathrm{A}$ & 213 \\
\hline & & & & 221.141 & $\mathrm{HCOOCH}_{3(v=0)}\left(18_{13,6} \rightarrow 17_{13,5}\right) \mathrm{A}$ & 213 \\
\hline \multirow[t]{4}{*}{229.056} & $\mathrm{CH}_{3} \mathrm{COCH}_{3(v=0)}\left(22_{1,21} \rightarrow 21_{2,20}\right) \mathrm{EE}$ & 133 & $*$ & 229.056 & $\mathrm{CH}_{3} \mathrm{COCH}_{3(v=0)}\left(22_{1,21} \rightarrow 21_{1,20}\right) \mathrm{EE}$ & 133 \\
\hline & & & & 229.056 & $\mathrm{CH}_{3} \mathrm{COCH}_{3(v=0)}\left(22_{2,21} \rightarrow 21_{2,20}\right) \mathrm{EE}$ & 133 \\
\hline & & & & 229.056 & $\mathrm{CH}_{3} \mathrm{COCH}_{3(v=0)}\left(22_{2,21} \rightarrow 21_{1,20}\right) \mathrm{EE}$ & 133 \\
\hline & & & & 229.058 & $\mathrm{CH}_{3} \mathrm{COCH}_{3(v=0)}\left(14_{9,6} \rightarrow 13_{8,5}\right) \mathrm{EE}$ & 85 \\
\hline 229.078 & $\mathrm{CH}_{3} \mathrm{COCH}_{3(v=0)}\left(22_{1,21} \rightarrow 21_{2,20}\right) \mathrm{AA}$ & 133 & $*$ & 229.078 & $\mathrm{CH}_{3} \mathrm{COCH}_{3(v=0)}\left(22_{2,21} \rightarrow 21_{1,20}\right) \mathrm{AA}$ & 133 \\
\hline 229.087 & $\mathrm{CH}_{2} \mathrm{CHCN}_{(v=0)}\left(24_{3,21} \rightarrow 23_{3,20}\right)$ & 156 & & & & \\
\hline 229.117 & $\mathrm{HCOOCH}_{3(v=1)}\left(19_{3,17} \rightarrow 18_{2,16}\right) \mathrm{E}$ & 303 & & & & \\
\hline 229.127 & $\mathrm{CH}_{3} \mathrm{COCH}_{3(v=0)}\left(12_{10,2} \rightarrow 11_{9,2}\right) \mathrm{EE}$ & 68 & & & & \\
\hline 229.134 & $\mathrm{CH}_{3} \mathrm{CHO}\left(9_{3,7} \rightarrow 9_{2,7}\right)$ & 62 & & & & \\
\hline 229.203 & UL & & $?$ & & & \\
\hline 229.224 & $\mathrm{HCOOCH}_{3(v=0)}\left(23_{9,14} \rightarrow 23_{8,15}\right) \mathrm{A}$ & 217 & & & & \\
\hline 229.259 & $\mathrm{HCOOCH}_{3(v=0)}\left(23_{9,14} \rightarrow 23_{8,15}\right) \mathrm{E}$ & 217 & $*$ & 229.258 & $\mathrm{CH}_{3} \mathrm{CHO}\left(10_{3,7} \rightarrow 10_{2,8}\right)$ & 71 \\
\hline 229.265 & $\mathrm{CH}_{3} \mathrm{CH}_{2} \mathrm{CN}_{(v=0)}\left(26_{2,25} \rightarrow 25_{2,24}\right)$ & 154 & $\ddagger$ & & & \\
\hline 229.320 & $\mathrm{HCOOCH}_{3(v=0)}\left(23_{9,15} \rightarrow 23_{8,16}\right) \mathrm{E}$ & 217 & & & & \\
\hline 229.348 & $\mathrm{SO}_{2(v=0)}\left(11_{5,7} \rightarrow 12_{4,8}\right)$ & 122 & $\dagger$ & & & \\
\hline 229.389 & $\mathrm{HCOOCH}_{3(v=0)}\left(23_{9,15} \rightarrow 23_{8,16}\right) \mathrm{A}$ & 217 & & & & \\
\hline 229.405 & $\mathrm{HCOOCH}_{3(v=0)}\left(18_{3,15} \rightarrow 17_{3,14}\right) \mathrm{E}$ & 111 & & & & \\
\hline 229.420 & $\mathrm{HCOOCH}_{3(v=0)}\left(18_{3,15} \rightarrow 17_{3,14}\right) \mathrm{A}$ & 111 & & & & \\
\hline 229.474 & $\mathrm{HCOOCH}_{3(v=0)}\left(20_{3,17} \rightarrow 19_{4,16}\right) \mathrm{E}$ & 134 & & & & \\
\hline \multirow[t]{2}{*}{229.491} & $\mathrm{t}-\mathrm{CH}_{3} \mathrm{CH}_{2} \mathrm{OH}\left(17_{5,12} \rightarrow 17_{4,13}\right)$ & 160 & $*$ & \multirow{2}{*}{\multicolumn{2}{|c|}{ 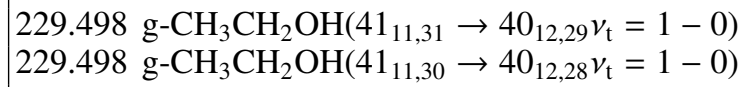 }} & 925 \\
\hline & & & & & & 925 \\
\hline 229.505 & $\mathrm{HCOOCH}_{3(v=0)}\left(20_{3,17} \rightarrow 19_{4,16}\right) \mathrm{A}$ & 134 & & & & \\
\hline 229.539 & $\mathrm{HCOOCH}_{3(v=0)}\left(31_{5,27} \rightarrow 31_{3,28}\right) \mathrm{E}$ & 309 & & & & \\
\hline 229.589 & $\mathrm{CH}_{3} \mathrm{OH}_{\left(v_{\mathrm{t}}=0\right)}\left(15_{4,11} \rightarrow 16_{3,13}\right) \mathrm{E}$ & 374 & & & & \\
\hline 229.607 & $\mathrm{HCOOCH}_{3(v=0)}\left(31_{5,27} \rightarrow 31_{3,28}\right) \mathrm{A}$ & 309 & & & & \\
\hline 229.648 & $\mathrm{CH}_{2} \mathrm{CHCN}_{(v=0)}\left(25_{1,25} \rightarrow 24_{1,24}\right)$ & 146 & & & & \\
\hline 229.661 & $\mathrm{HCOOCH}_{3(v=1)}\left(25_{9,16} \rightarrow 25_{8,17}\right) \mathrm{A}$ & 432 & & & & \\
\hline 229.759 & $\mathrm{CH}_{3} \mathrm{OH}_{\left(v_{\mathrm{t}}=0\right)}\left(8_{-1,8} \rightarrow 7_{0,7}\right) \mathrm{E}$ & 89 & $\ddagger$ & & & \\
\hline 229.858 & ${ }^{34} \mathrm{SO}_{2(v=0)}\left(4_{2,2} \rightarrow 3_{1,3}\right)$ & 19 & & & & \\
\hline 229.864 & $\mathrm{CH}_{3} \mathrm{OH}_{\left(v_{\mathrm{t}}=0\right)}\left(19_{5,15} \rightarrow 20_{4,16}\right) \mathrm{A}++$ & 578 & & & & \\
\hline 229.888 & ${ }^{34} \mathrm{SO}_{2(v=0)}\left(17_{3,15} \rightarrow 18_{0,18}\right)$ & 162 & & & & \\
\hline 229.939 & $\mathrm{CH}_{3} \mathrm{OH}_{\left(v_{\mathrm{t}}=0\right)}\left(19_{5,14} \rightarrow 20_{4,17}\right) \mathrm{A}--$ & 578 & & & & \\
\hline 229.987 & ${ }^{34} \mathrm{SO}_{2(v=0)}\left(42_{6,36} \rightarrow 41_{7,35}\right)$ & 921 & & & & \\
\hline 230.027 & $\mathrm{CH}_{3} \mathrm{OH}_{\left(v_{\mathrm{t}}=0\right)}\left(3_{-2,2} \rightarrow 4_{-1,4}\right) \mathrm{E}$ & 40 & & & & \\
\hline 230.109 & $\mathrm{C}_{6} \mathrm{H}(\mathrm{J}=165 / 2-163 / 2, \Omega=1 / 2,1=\mathrm{f})$ & 482 & $?^{\ddagger}$ & & & \\
\hline 230.140 & $\mathrm{CH}_{3} \mathrm{OCH}_{3}\left(25_{4,22} \rightarrow 25_{3,22}\right) \mathrm{EA}$ & 319 & $\mathrm{~A}(\mathrm{E}) \mathrm{A}(\mathrm{E})^{\ddagger}$ & & & \\
\hline \multirow[t]{3}{*}{230.170} & $\mathrm{CH}_{3} \mathrm{COCH}_{3(v=0)}\left(23_{0,23} \rightarrow 22_{1,22}\right) \mathrm{AE}$ & 135 & $*$ & 230.170 & $\mathrm{CH}_{3} \mathrm{COCH}_{3(v=0)}\left(23_{1,23} \rightarrow 22_{0,22}\right) \mathrm{AE}$ & 135 \\
\hline & & & & 230.170 & $\mathrm{CH}_{3} \mathrm{COCH}_{3(v=0)}\left(23_{0,23} \rightarrow 22_{0,22}\right) \mathrm{EA}$ & 135 \\
\hline & & & & 230.170 & $\mathrm{CH}_{3} \mathrm{COCH}_{3(v=0)}\left(23_{1,23} \rightarrow 22_{1,22}\right) \mathrm{EA}$ & 135 \\
\hline
\end{tabular}


Table A.1. continued.

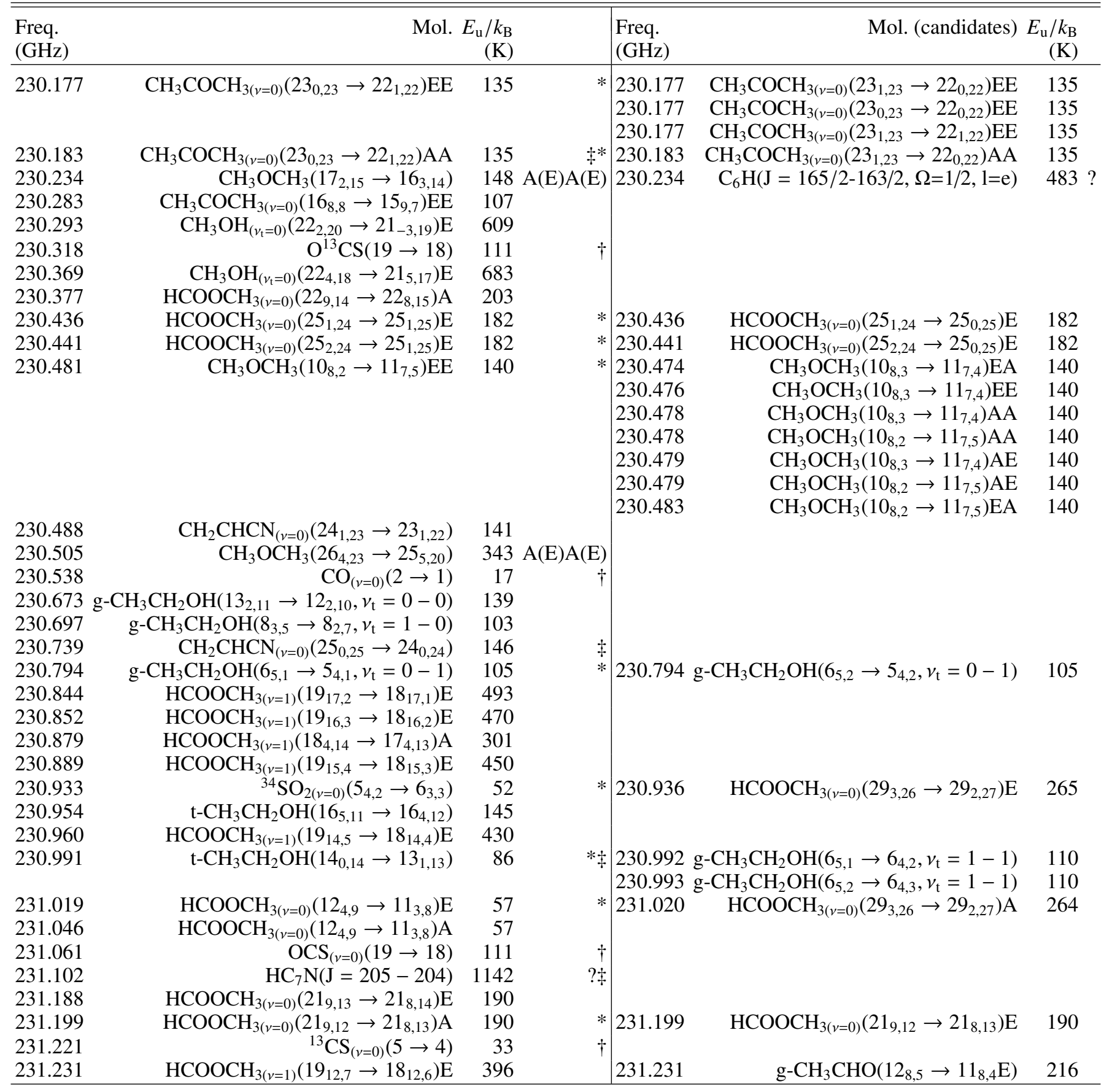


S. Feng et al.: Resolving the chemical substructure of Orion-KL

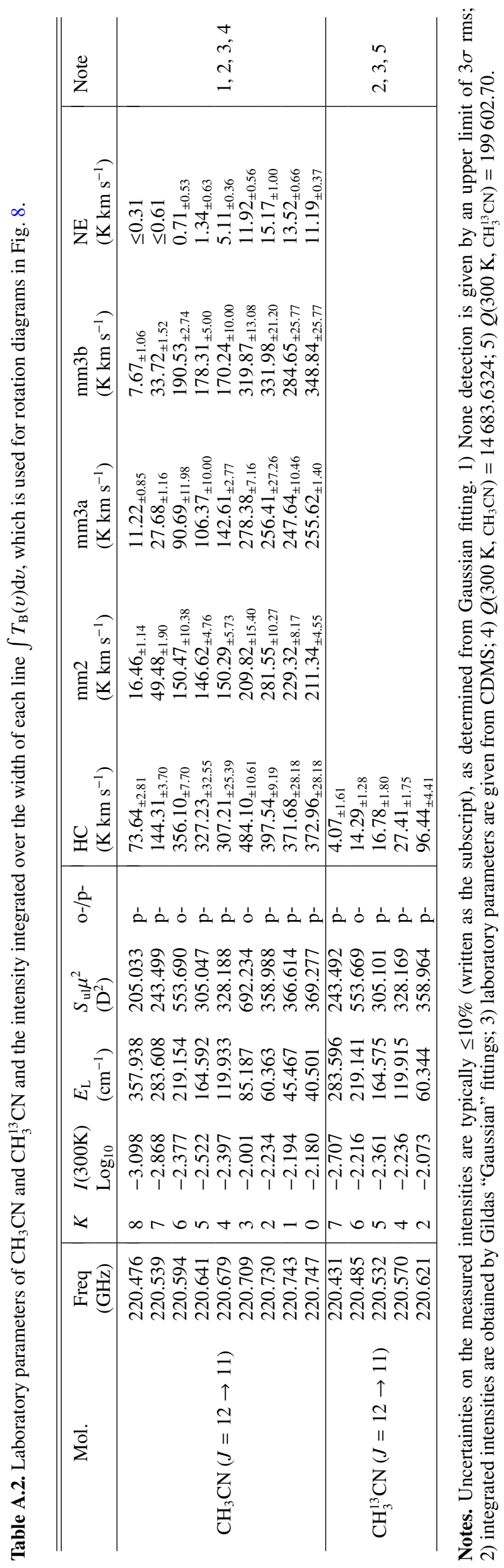




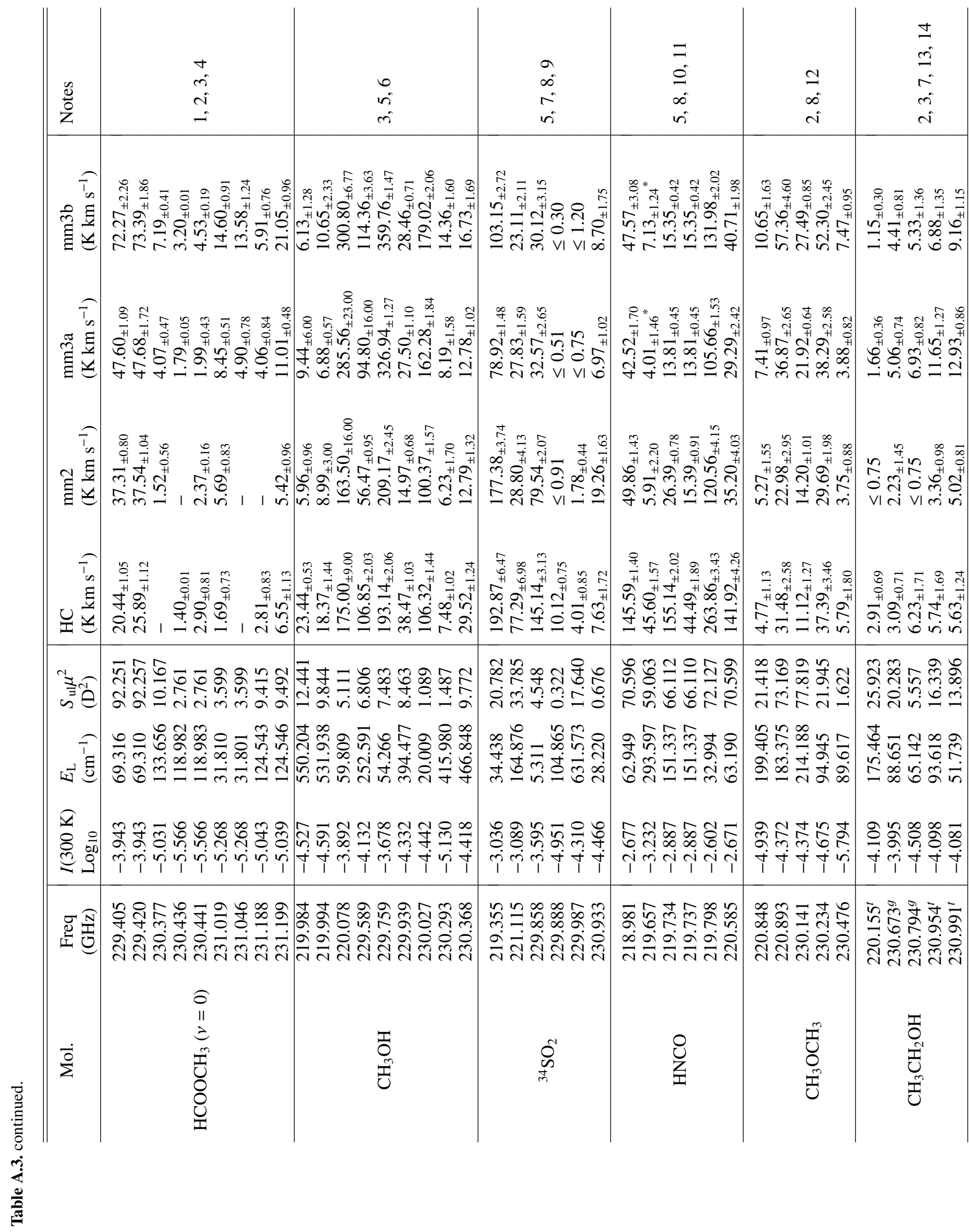




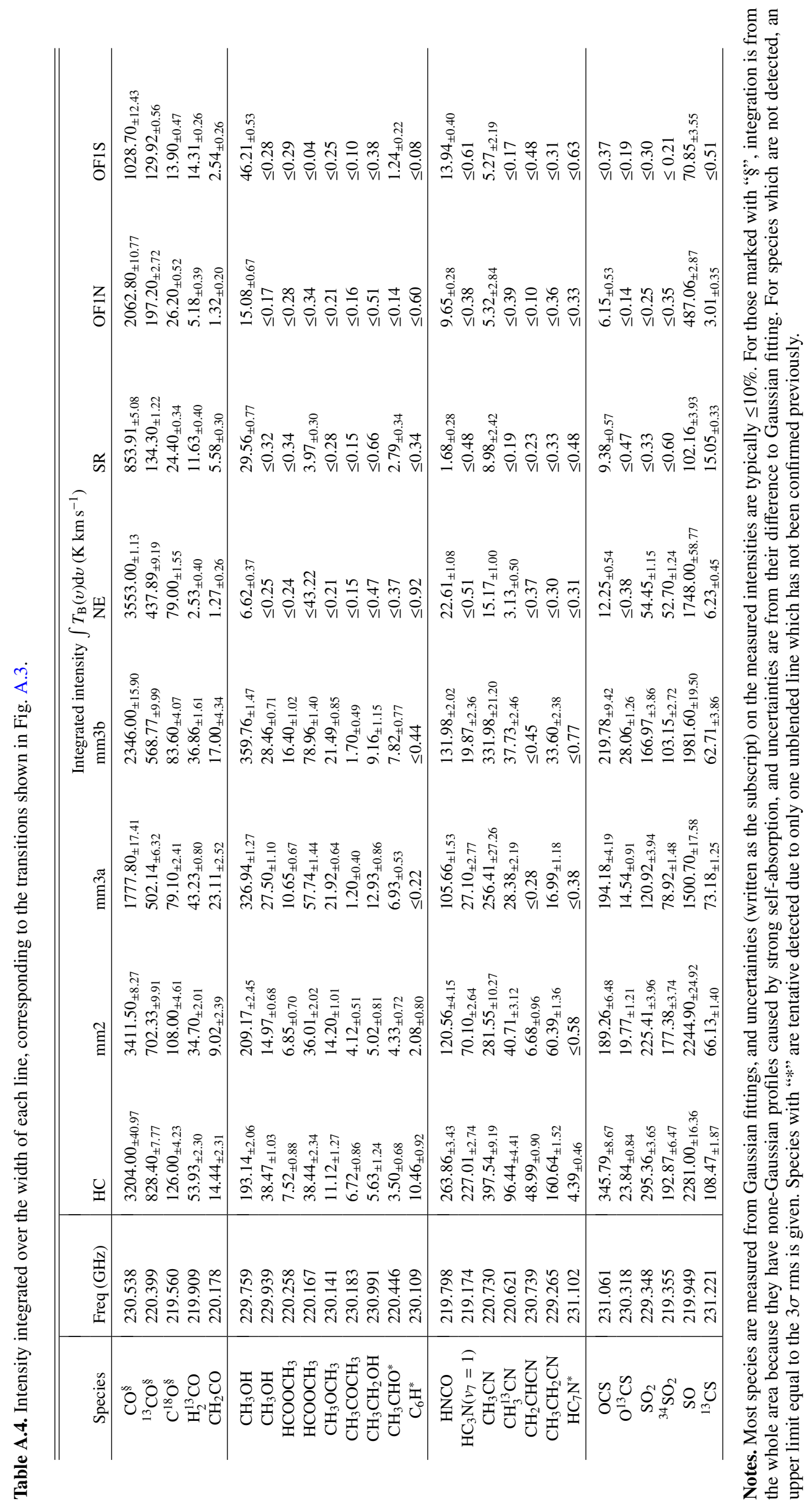


S. Feng et al.: Resolving the chemical substructure of Orion-KL

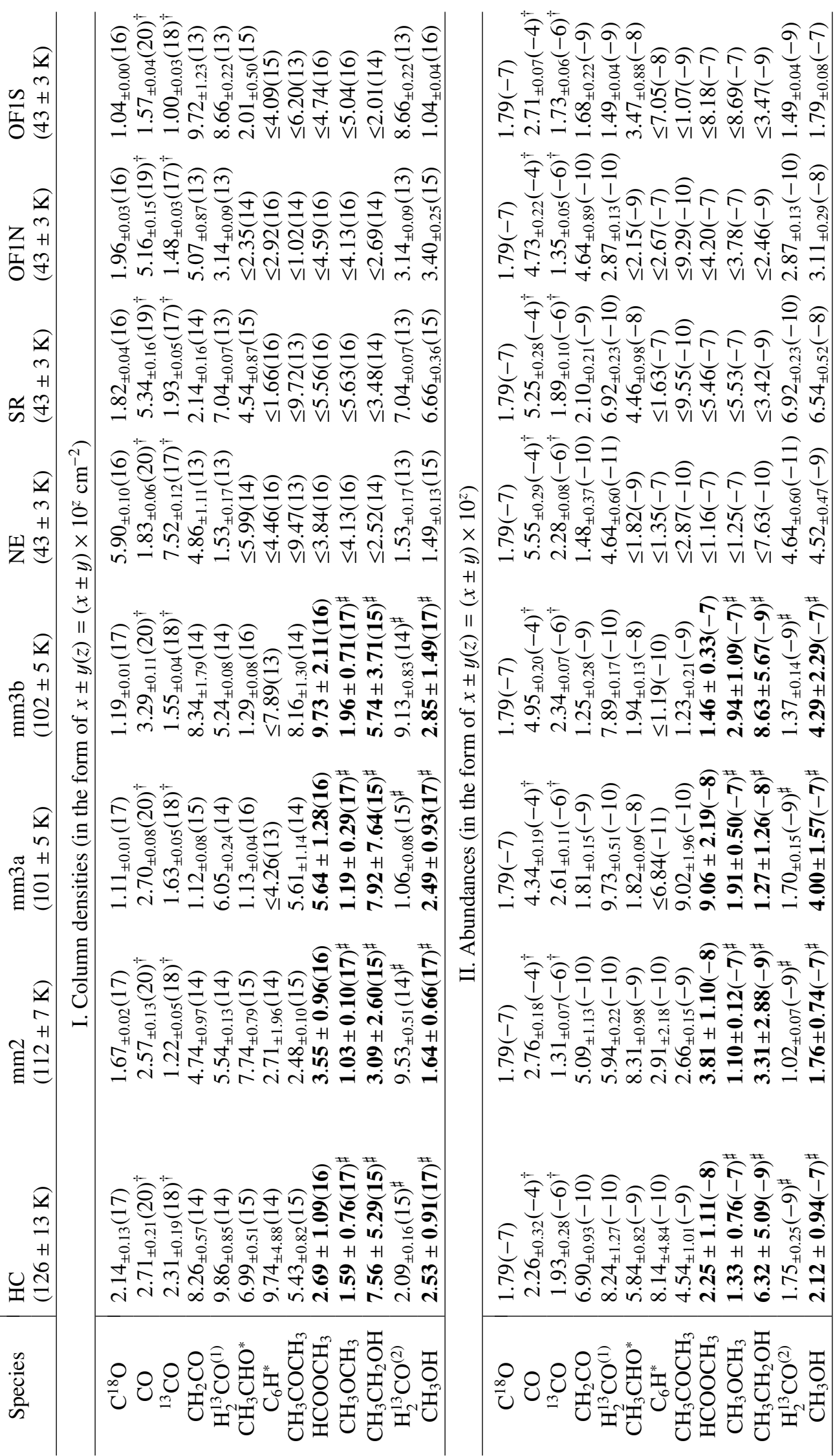

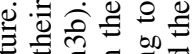

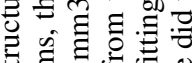

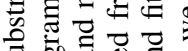
क 0 :

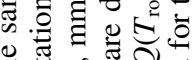
o 항

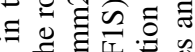
Oิ 局.

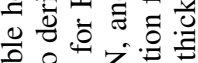

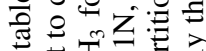

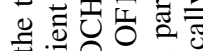

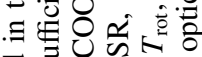
क का जा

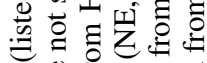
o

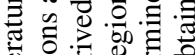
政.

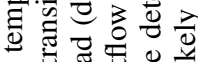

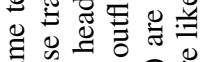
我 Ð

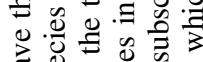
过

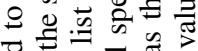

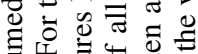

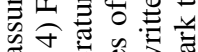

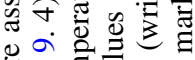
ob $\bar{g}$. +

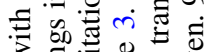

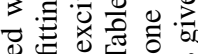
政

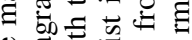

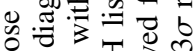

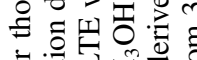
可

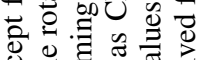
可

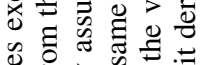
o 을

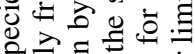

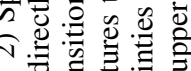

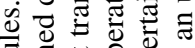

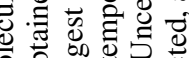
응

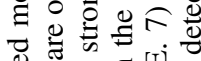
过 纪

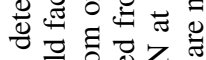

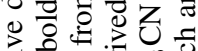

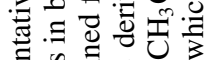

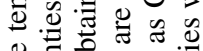

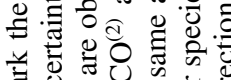

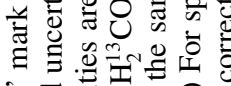

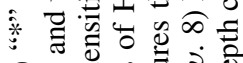

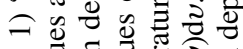

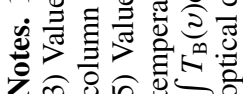

A71, page 35 of 49 


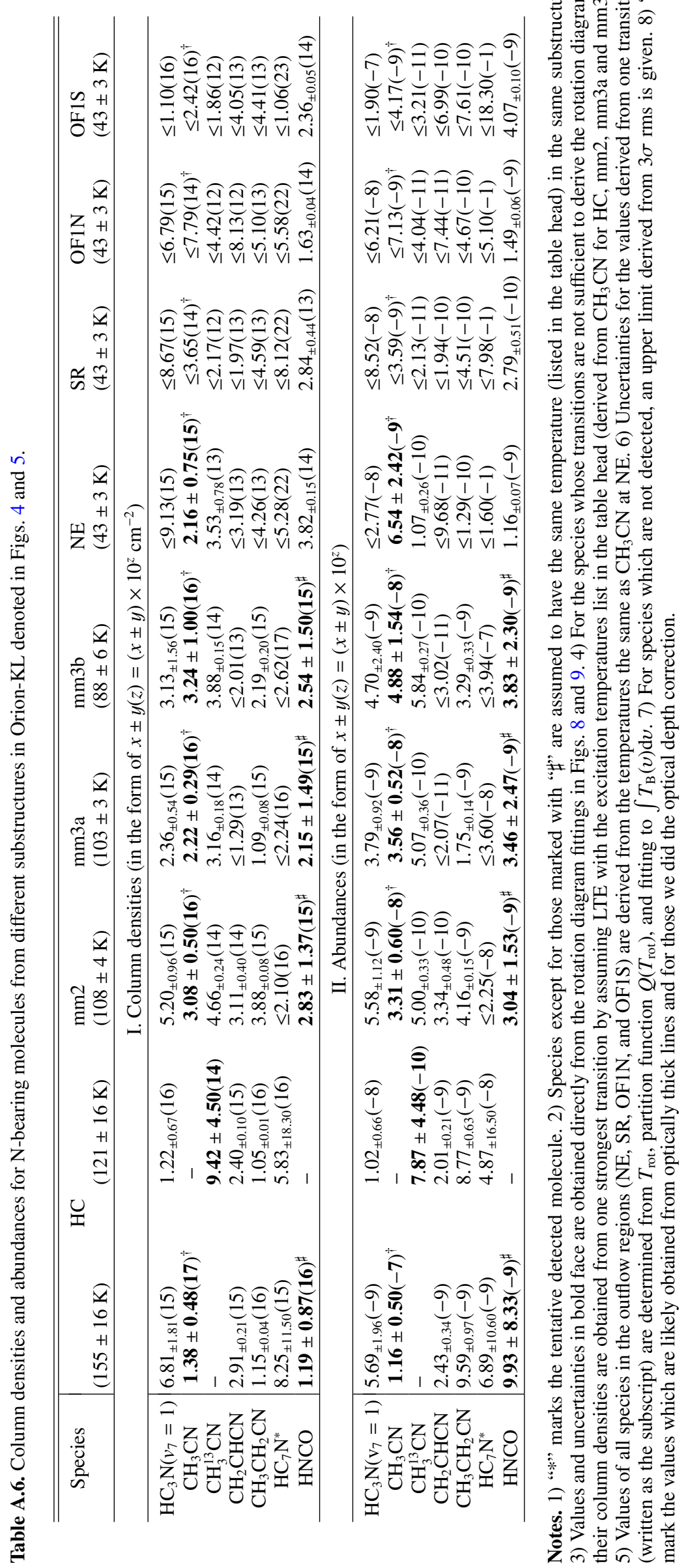


S. Feng et al.: Resolving the chemical substructure of Orion-KL

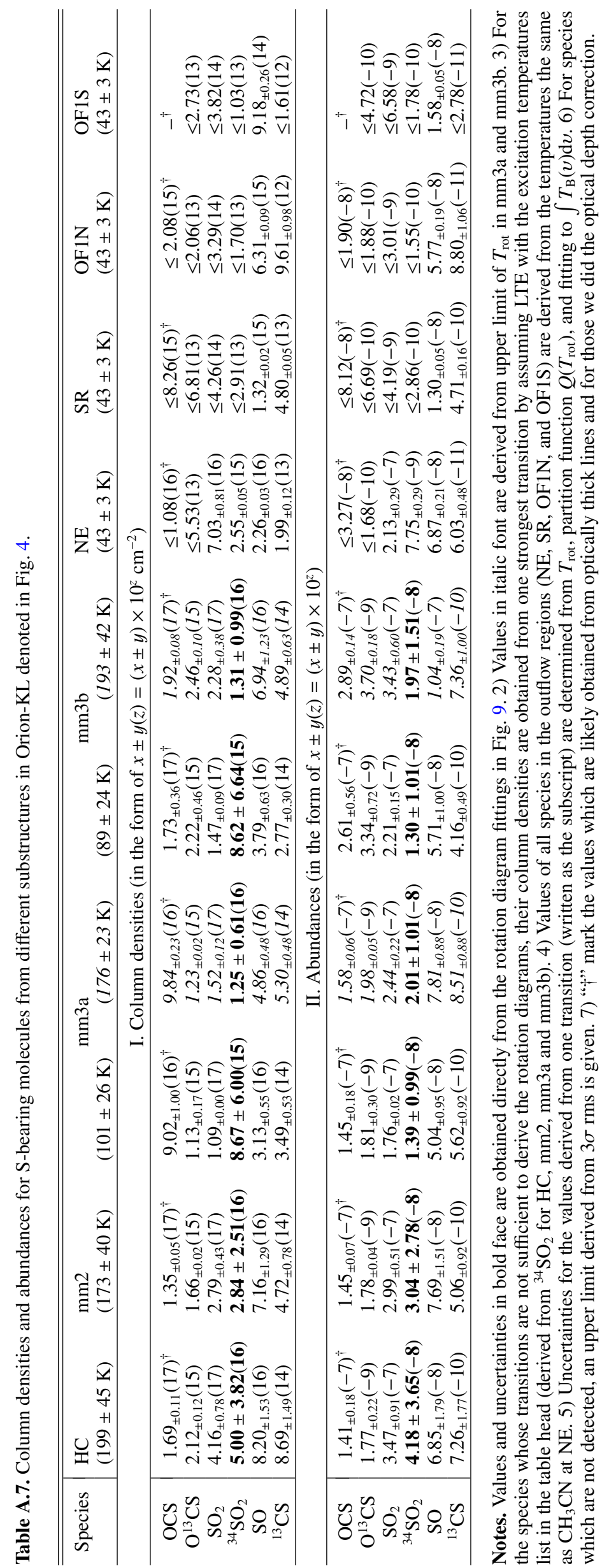



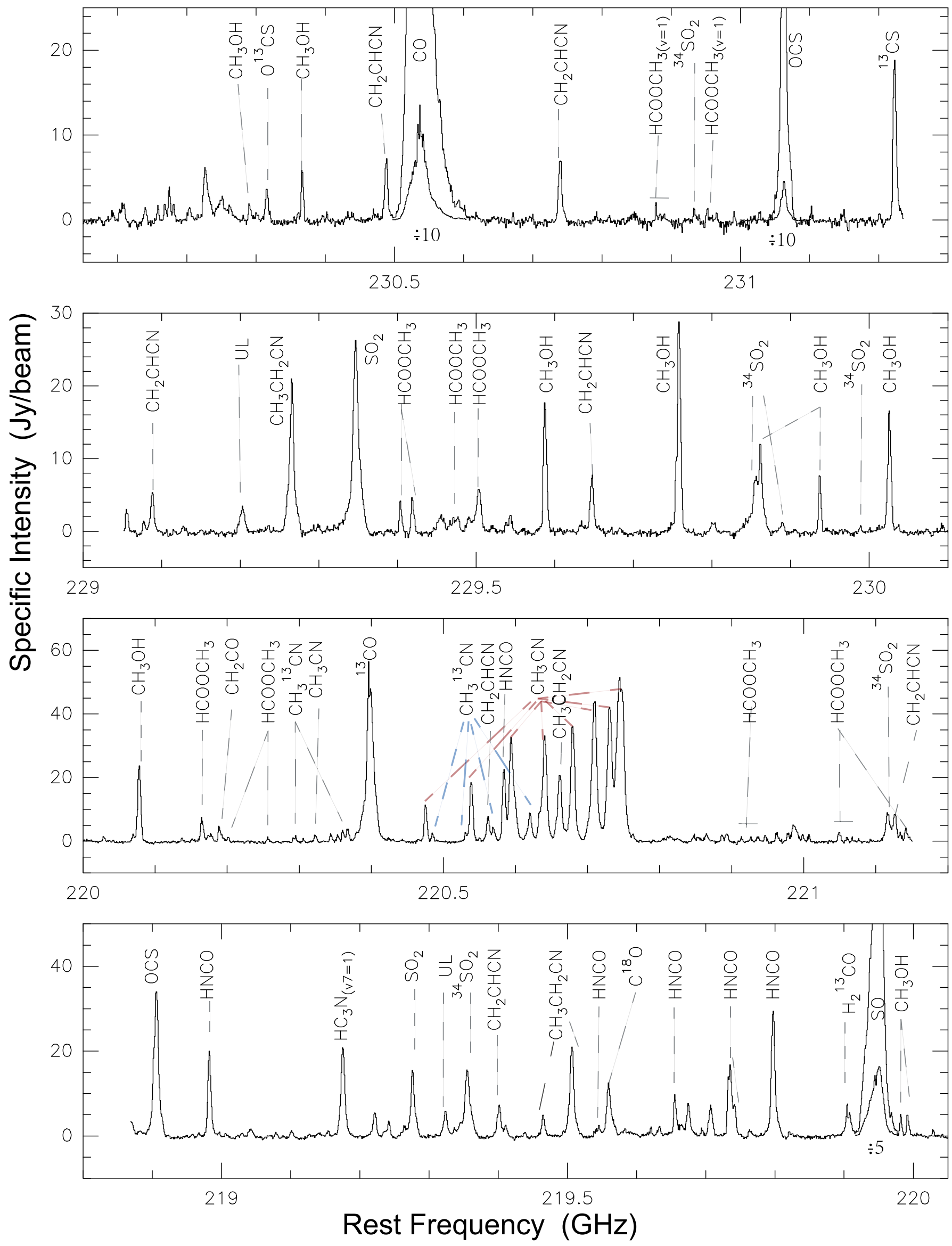

Fig. A.1. Spectrum from the $\mathrm{HC}$, with identified strong lines marked. From $220.5 \mathrm{GHz}$ to $220.8 \mathrm{GHz}, \mathrm{CH}_{3} \mathrm{CN}$ and $\mathrm{CH}_{3}^{13} \mathrm{CN}_{\text {are }}$ labelled in red and blue, respectively. COMs containing only $\mathrm{C}, \mathrm{H}, \mathrm{O}$ elements and other tentatively detections are labelled in Fig. A.2. 
S. Feng et al.: Resolving the chemical substructure of Orion-KL

$\mathrm{CH}_{3} \mathrm{OH} @ \mathrm{~mm} 3 \mathrm{a}\left(\mathrm{T}_{\mathrm{rot}}=160 \mathrm{~K} ; \mathrm{N}_{\mathrm{mol}}=2.5 \times 10^{17} \mathrm{~cm}^{-2} ; \mathrm{V}_{\mathrm{lsr}}=7.6 \mathrm{~km} \mathrm{~s}^{-1} ; \Delta \mathrm{V}=5 \mathrm{~km} \mathrm{~s}^{-1}\right)$
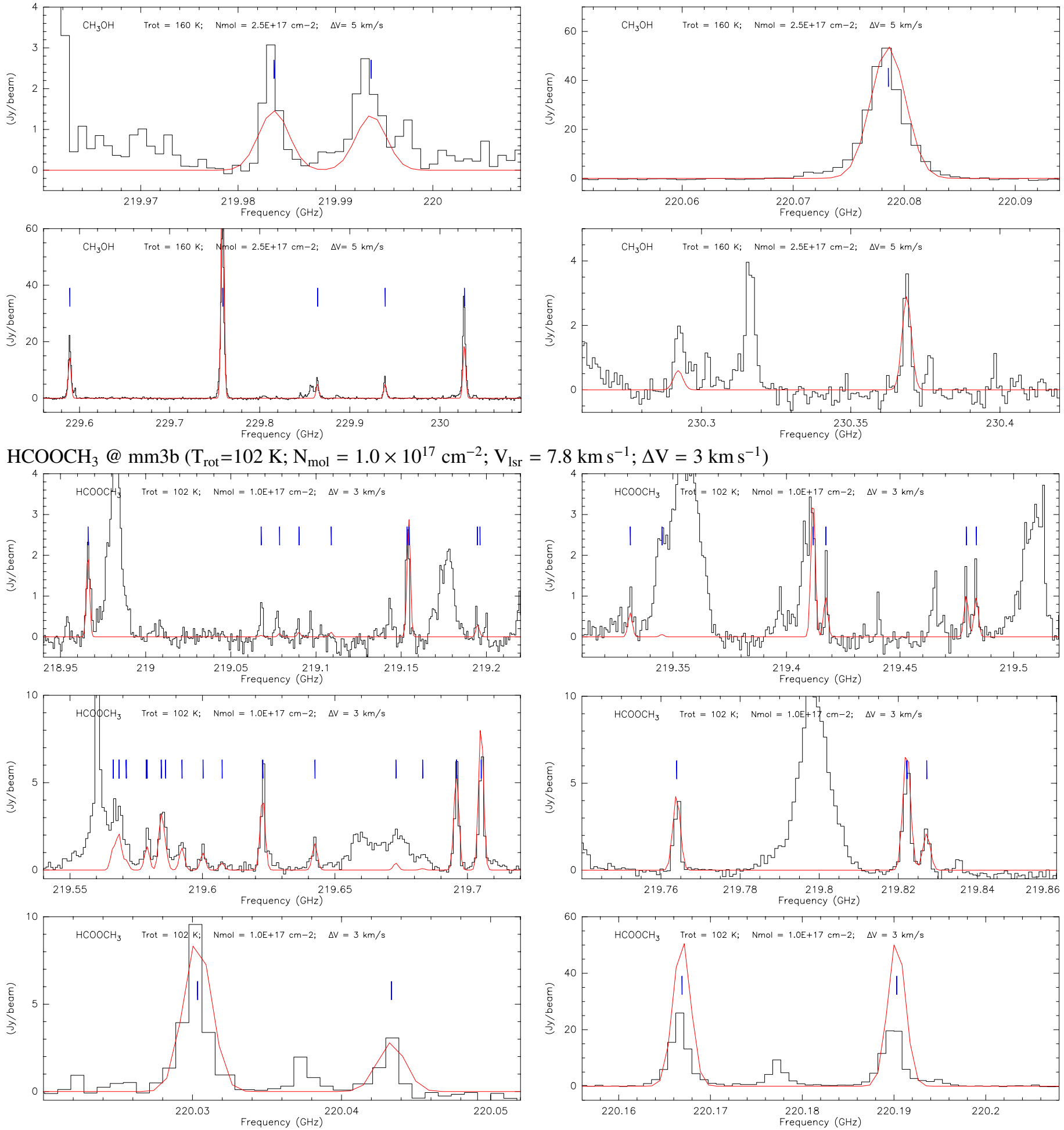

Fig. A.2. Synthetic spectrum fitting of COMs towards their emission peaks ( $V_{\mathrm{lsr}}$ of each species are corrected according to $V_{\text {peak }}$ from Fig. A.3). The black histograms are the observed spectra, overlaid with a best fit model spectrum for given uniform FWHM linewidth $(\triangle V$ ) (fit from Fig. A.3), $T_{\text {rot }}$ (assumed from an adjacent substructure), and $N_{\text {mol }}$ (adjusted based on $N_{\mathrm{T}}$ in Tables A.5I-A.7I) listed on top of each panel. Blue lines mark the central frequency of each transition. Uncertainties of the fitting to observations come from the assumption that under LTE, the candidate molecule lines are optically thin, and each species has the same line width at different transitions. Referenced molecular data are from CDMS/JPL (Sanchez-Monge 2011; Palau et al. 2011). 
A\&A 581, A71 (2015)
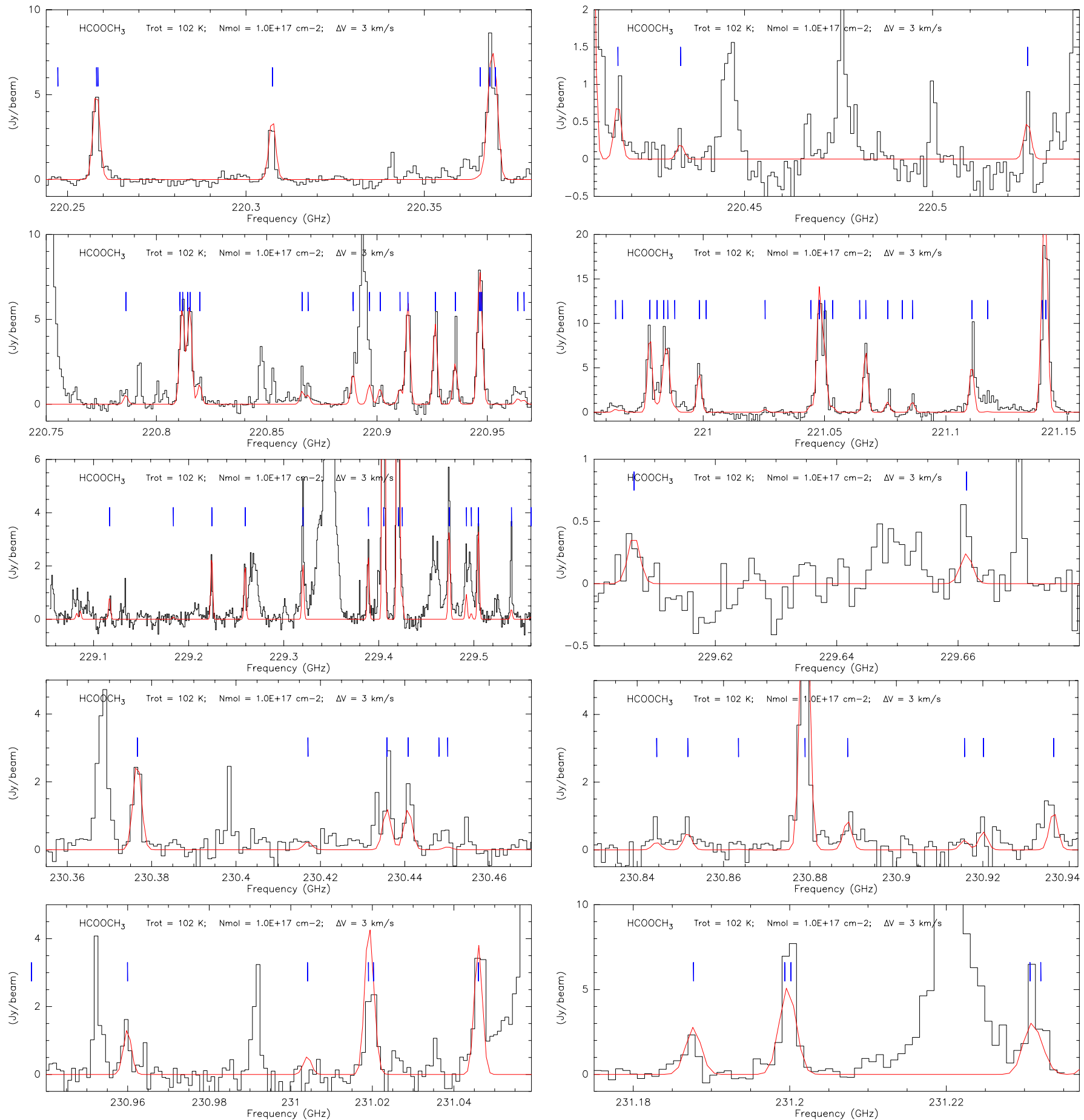

$\mathrm{CH}_{3} \mathrm{COCH}_{3} @ \mathrm{SMA} 1\left(\mathrm{~T}_{\mathrm{rot}}=126 \mathrm{~K} ; \mathrm{N}_{\mathrm{mol}}=1.5 \times 10^{16} \mathrm{~cm}^{-2} ; \mathrm{V}_{\mathrm{lsr}}=5.8 \mathrm{~km} \mathrm{~s}^{-1} ; \Delta \mathrm{V}=3 \mathrm{~km} \mathrm{~s}^{-1}\right)$
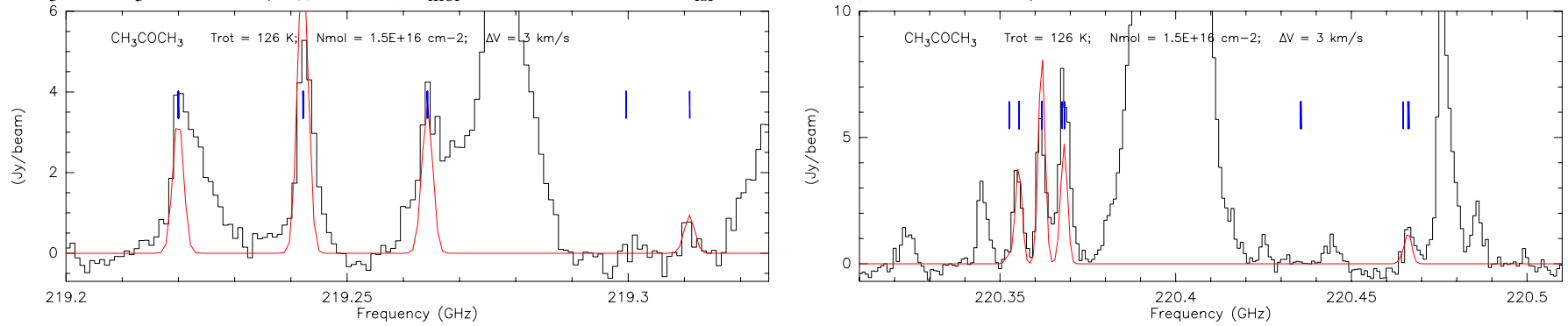

Fig. A.2. continued. 
S. Feng et al.: Resolving the chemical substructure of Orion-KL
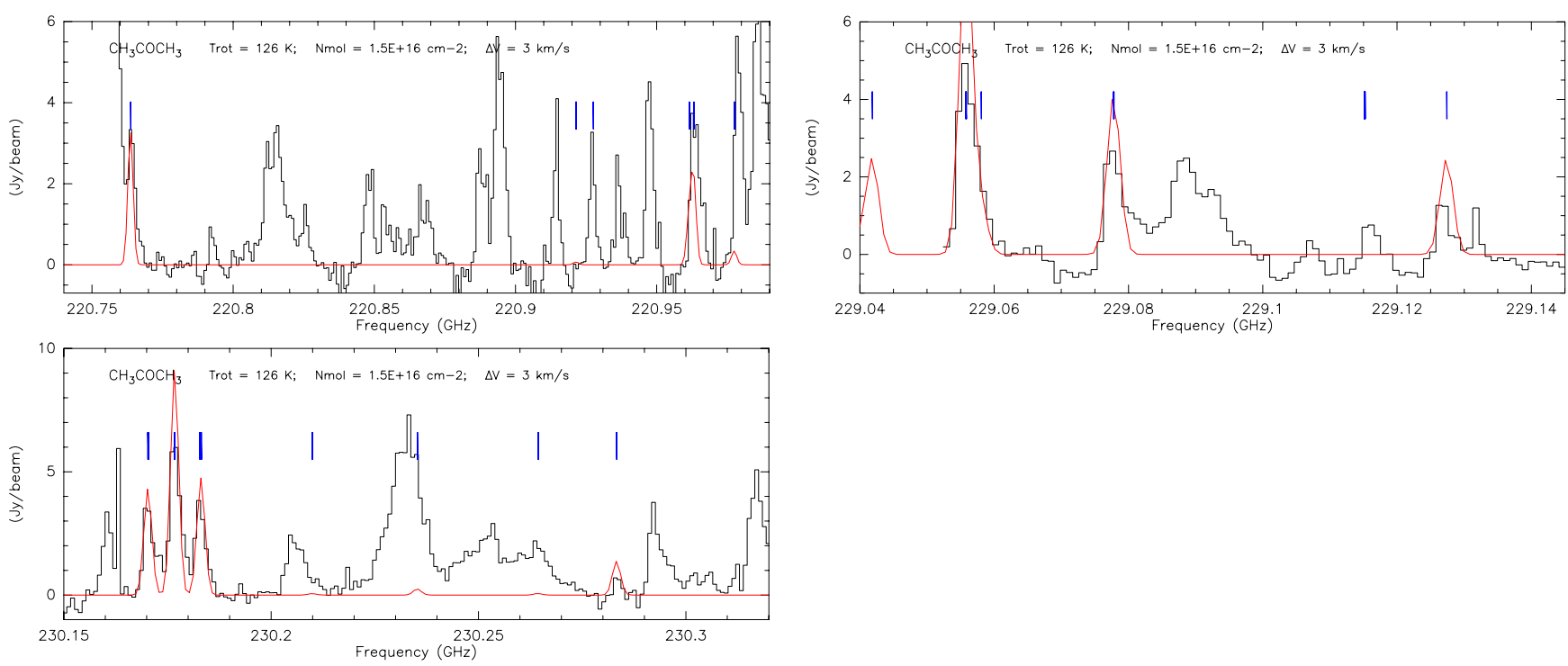

$\mathrm{CH}_{3} \mathrm{OCH}_{3} @ \mathrm{~mm} 3 \mathrm{~b}\left(\mathrm{~T}_{\mathrm{rot}}=92 \mathrm{~K} ; \mathrm{N}_{\mathrm{mol}}=2.0 \times 10^{17} \mathrm{~cm}^{-2} ; \mathrm{V}_{\mathrm{lsr}}=5.6 \mathrm{~km} \mathrm{~s}^{-1} ; \Delta \mathrm{V}=4 \mathrm{~km} \mathrm{~s}^{-1}\right)$
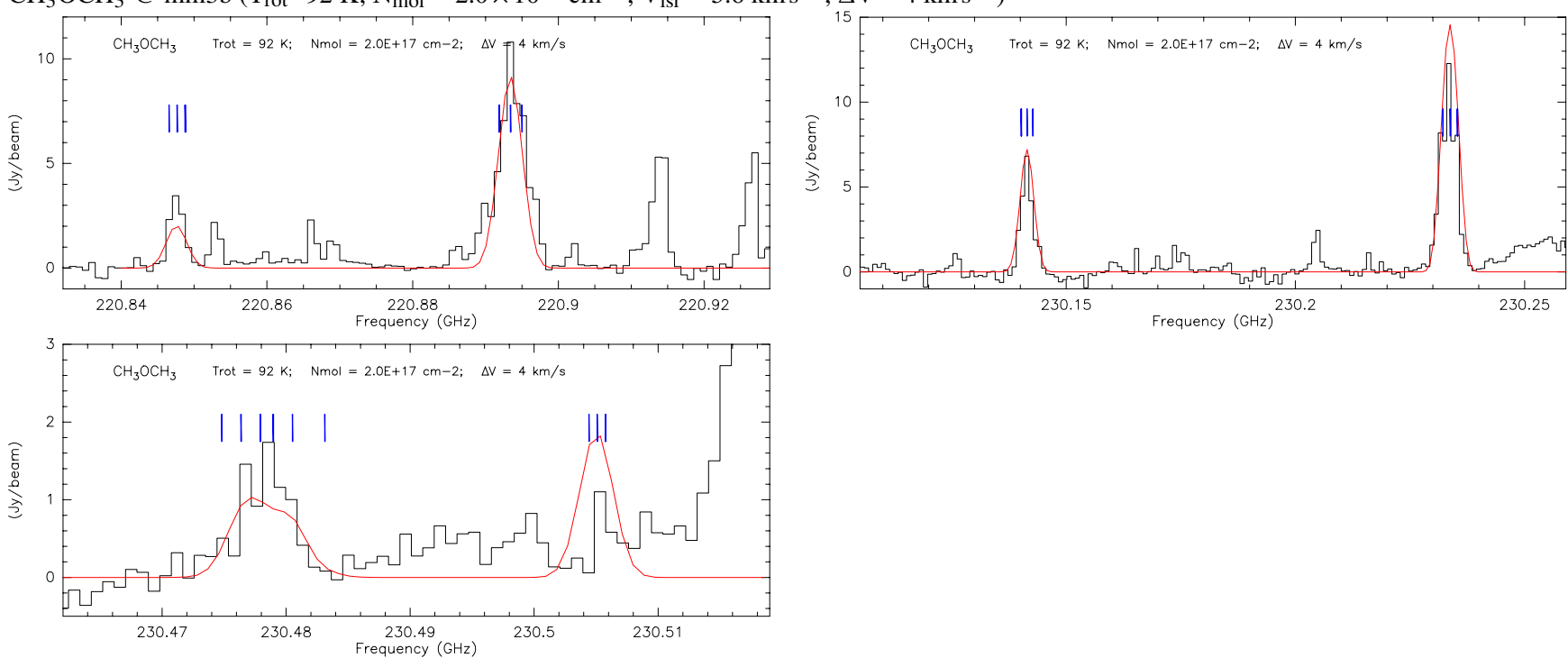

$\mathrm{CH}_{3} \mathrm{CH}_{2} \mathrm{OH} @$ near SMA1 \& mm3a $\left(\mathrm{T}_{\mathrm{rot}}=70 \mathrm{~K} ; \mathrm{N}_{\mathrm{mol}}=1.5 \times 10^{16} \mathrm{~cm}^{-2} ; \mathrm{V}_{1 \mathrm{sr}}=8.4 \mathrm{~km} \mathrm{~s}^{-1} ; \Delta \mathrm{V}=2.5 \mathrm{~km} \mathrm{~s}^{-1}\right)$
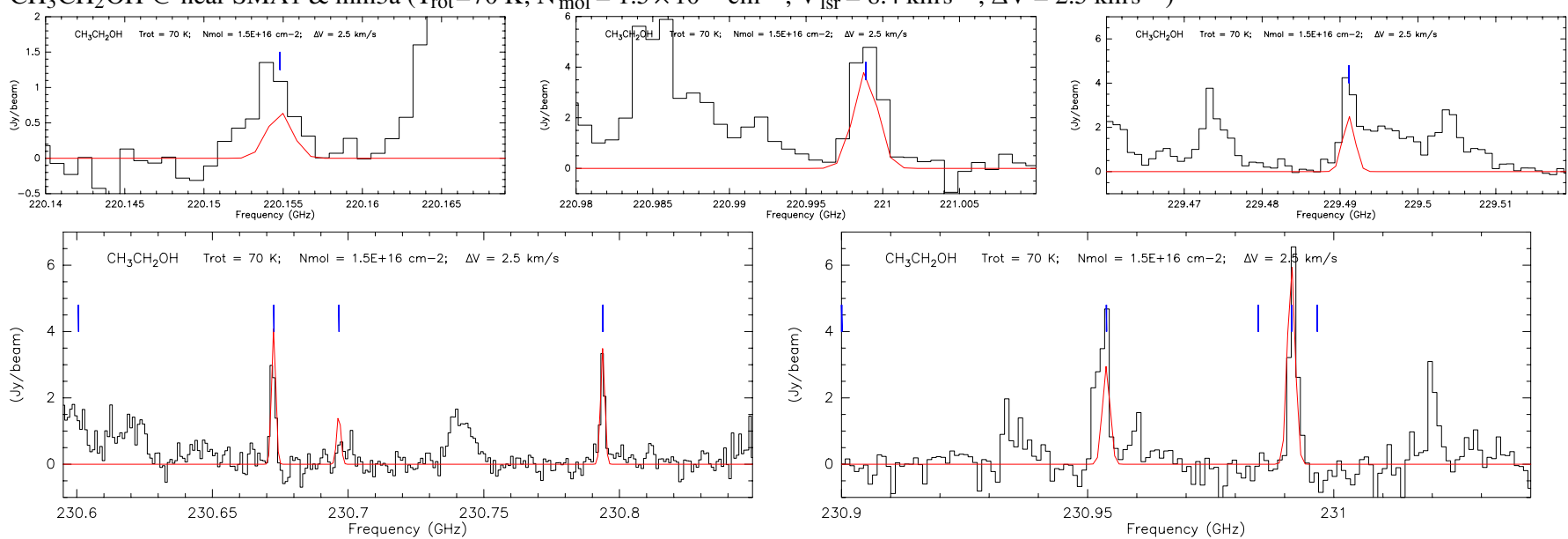

Fig. A.2. continued. 
$\mathrm{CH}_{2} \mathrm{CHCN} @$ North to hotcore $\left(\mathrm{T}_{\mathrm{rot}}=155 \mathrm{~K} ; \mathrm{N}_{\mathrm{mol}}=5.0 \times 10^{15} \mathrm{~cm}^{-2} ; \mathrm{V}_{\mathrm{lsr}}=4.9 \mathrm{~km} \mathrm{~s}^{-1} ; \Delta \mathrm{V}=7 \mathrm{~km} \mathrm{~s}^{-1}\right)$

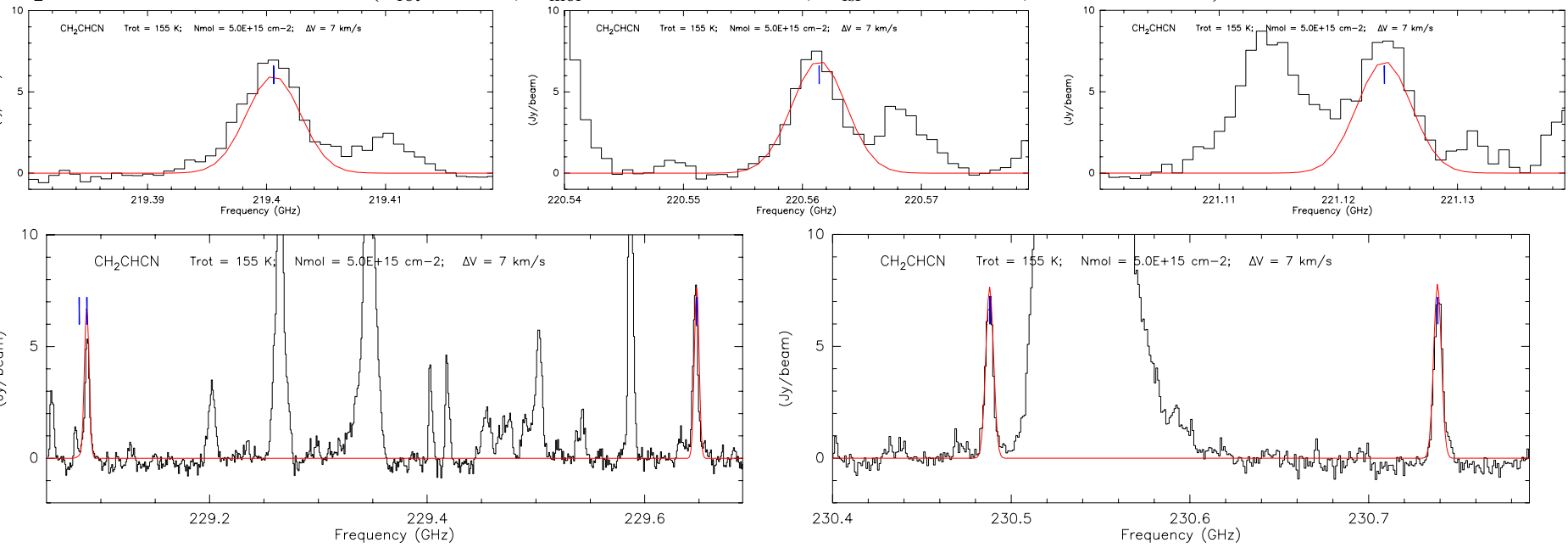

$\mathrm{CH}_{3} \mathrm{CH}_{2} \mathrm{CN} @$ hotcore $\left(\mathrm{T}_{\mathrm{rot}}=155 \mathrm{~K} ; \mathrm{N}_{\mathrm{mol}}=1.5 \times 10^{16} \mathrm{~cm}^{-2} ; \mathrm{V}_{\mathrm{lsr}}=5.1 \mathrm{~km} \mathrm{~s}^{-1} ; \Delta \mathrm{V}=9 \mathrm{~km} \mathrm{~s}^{-1}\right)$
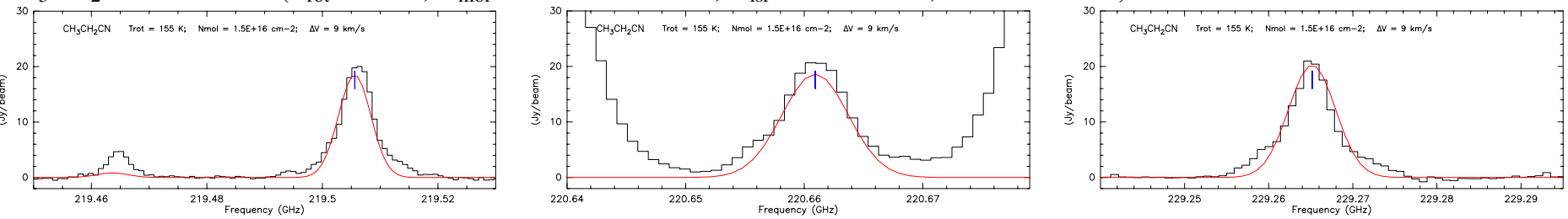

$\mathrm{CH}_{3} \mathrm{CHO} @ \mathrm{~mm} 3 \mathrm{~b}\left(\mathrm{~T}_{\mathrm{rot}}=102 \mathrm{~K} ; \mathrm{N}_{\mathrm{mol}}=1.3 \times 10^{16} \mathrm{~cm}^{-2} ; \mathrm{V}_{\mathrm{lsr}}=8.0 \mathrm{~km} \mathrm{~s}^{-1} ; \Delta \mathrm{V}=5 \mathrm{~km} \mathrm{~s}^{-1}\right)$
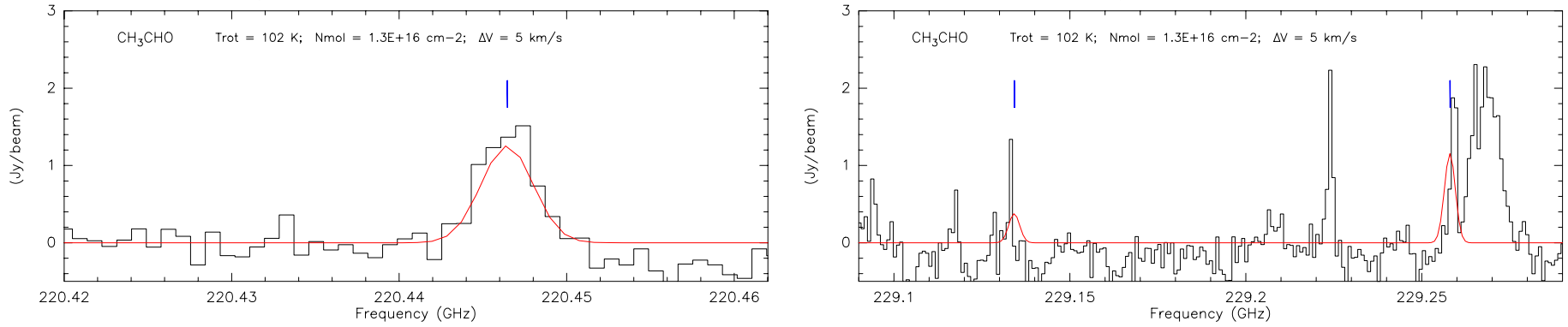

$\mathrm{C}_{6} \mathrm{H} @ \operatorname{SMA} 1\left(\mathrm{~T}_{\mathrm{rot}}=126 \mathrm{~K} ; \mathrm{N}_{\mathrm{mol}}=1.0 \times 10^{15} \mathrm{~cm}^{-2} ; \mathrm{V}_{\mathrm{lsr}}=4.0 \mathrm{~km} \mathrm{~s}^{-1} ; \Delta \mathrm{V}=4 \mathrm{~km} \mathrm{~s}^{-1}\right)$
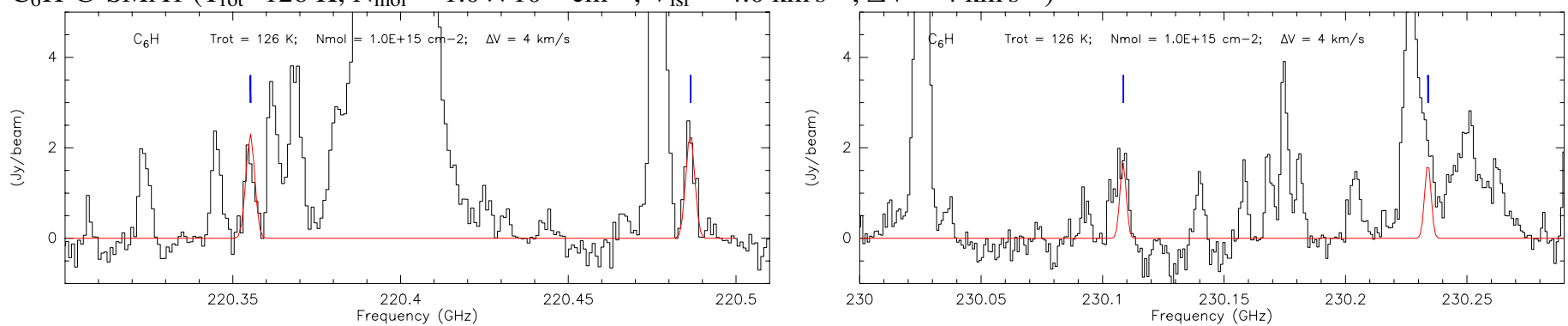

$\mathrm{HC}_{7} \mathrm{~N} @$ North to hotcore $\left(\mathrm{T}_{\text {rot }}=155 \mathrm{~K} ; \mathrm{N}_{\mathrm{mol}}=1.0 \times 10^{16} \mathrm{~cm}^{-2} ; \mathrm{V}_{\mathrm{lsr}}=6.3 \mathrm{~km} \mathrm{~s}^{-1} ; \Delta \mathrm{V}=5 \mathrm{~km} \mathrm{~s}^{-1}\right)$
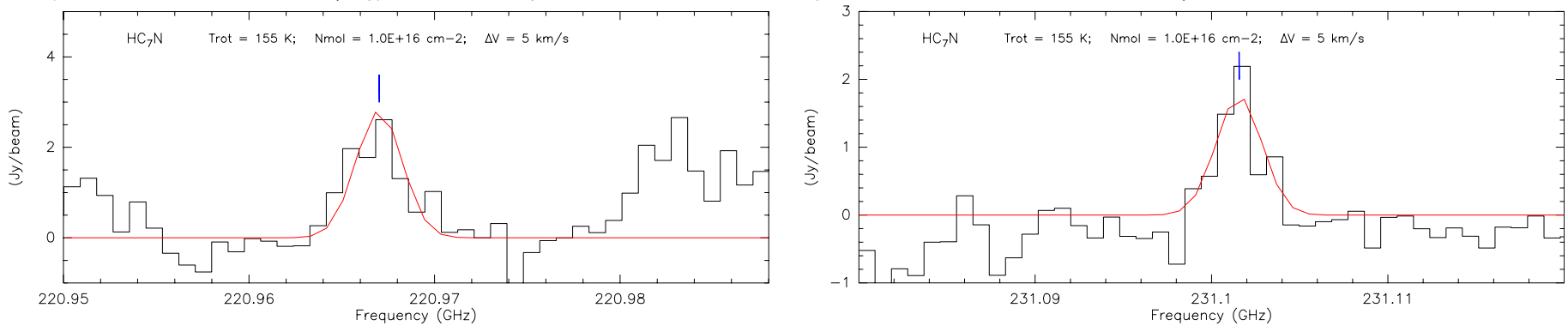

Fig. A.2. continued. 
S. Feng et al.: Resolving the chemical substructure of Orion-KL

\section{$(\mathrm{Jy} / \mathrm{beam})$}
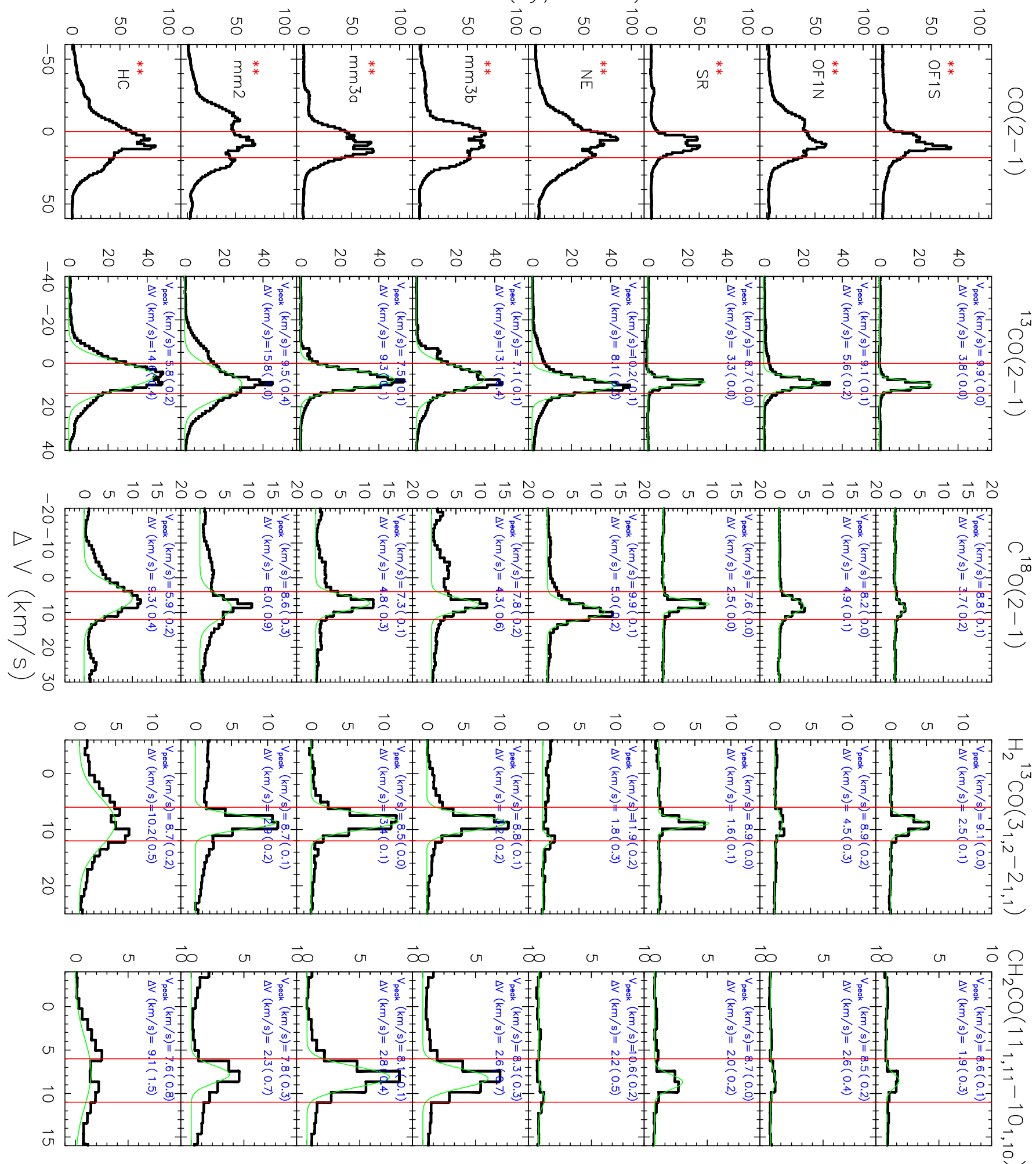

Fig. A.3. Line profiles of identified species from each continuum peak. In measuring the main $V_{\text {peak }}$ and $\Delta V$ of each line at each substructure, lines in green and notes in blue (in the form of "value(error)") are given by the Gaussian fittings using Gildas software. Multi-Gaussian fittings are used to $\mathrm{CH}_{3} \mathrm{CN}, \mathrm{CH}_{3}^{13} \mathrm{CN}, \mathrm{HCOOCH}_{3}(v=0)$, and $\mathrm{CH}_{3} \mathrm{COCH}_{3}$ due to blending, and to $\mathrm{SO}_{2},{ }^{34} \mathrm{SO}_{2}$, and $\mathrm{SO}$ due to the second velocity component (the second velocity component of HNCO measured from multi-Gaussian fittings are given in Fig. 7). Single Gaussian fittings are used to the rest lines, while lines whose $V_{\text {peak }}$ cannot be measured by Gaussian fittings owing to strong self-absorption are marked with "**”, and non-detections are not marked. Two red vertical lines in each panel mark the velocity range over which the emission from each species is integrated to make the distribution maps shown in Figs. 4 and 5. 
A\&A 581, A71 (2015)

\section{(Jy/beam)}
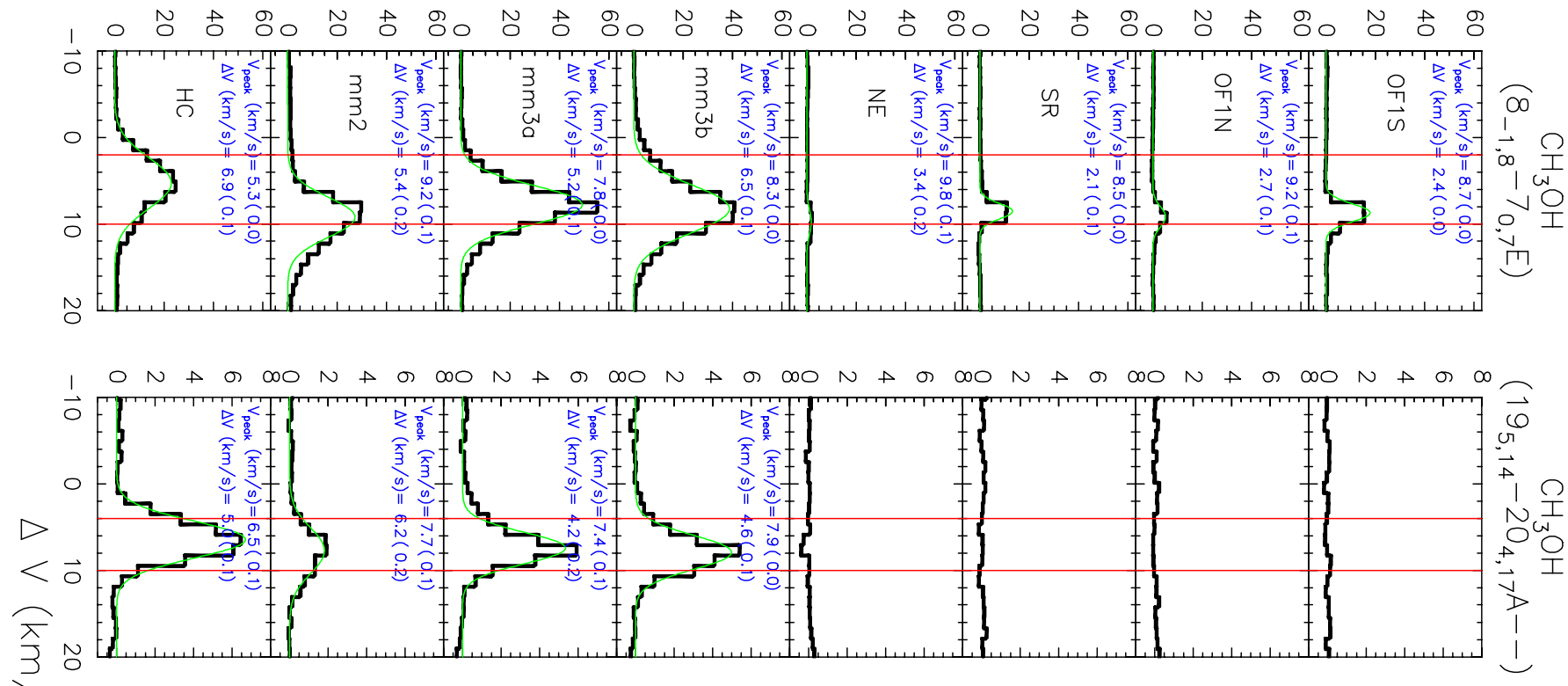

(s)
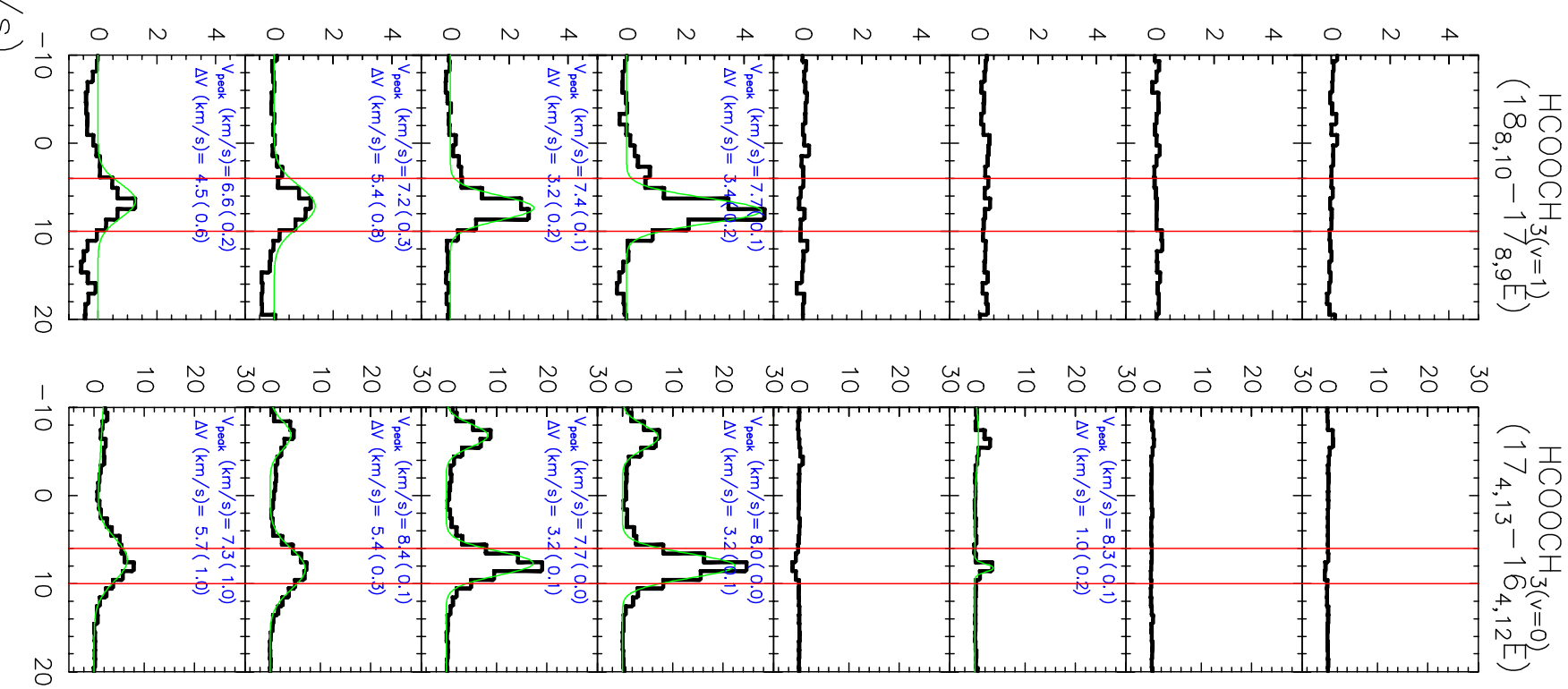

Fig. A.3. continued. 
S. Feng et al.: Resolving the chemical substructure of Orion-KL

(Jy/beam)
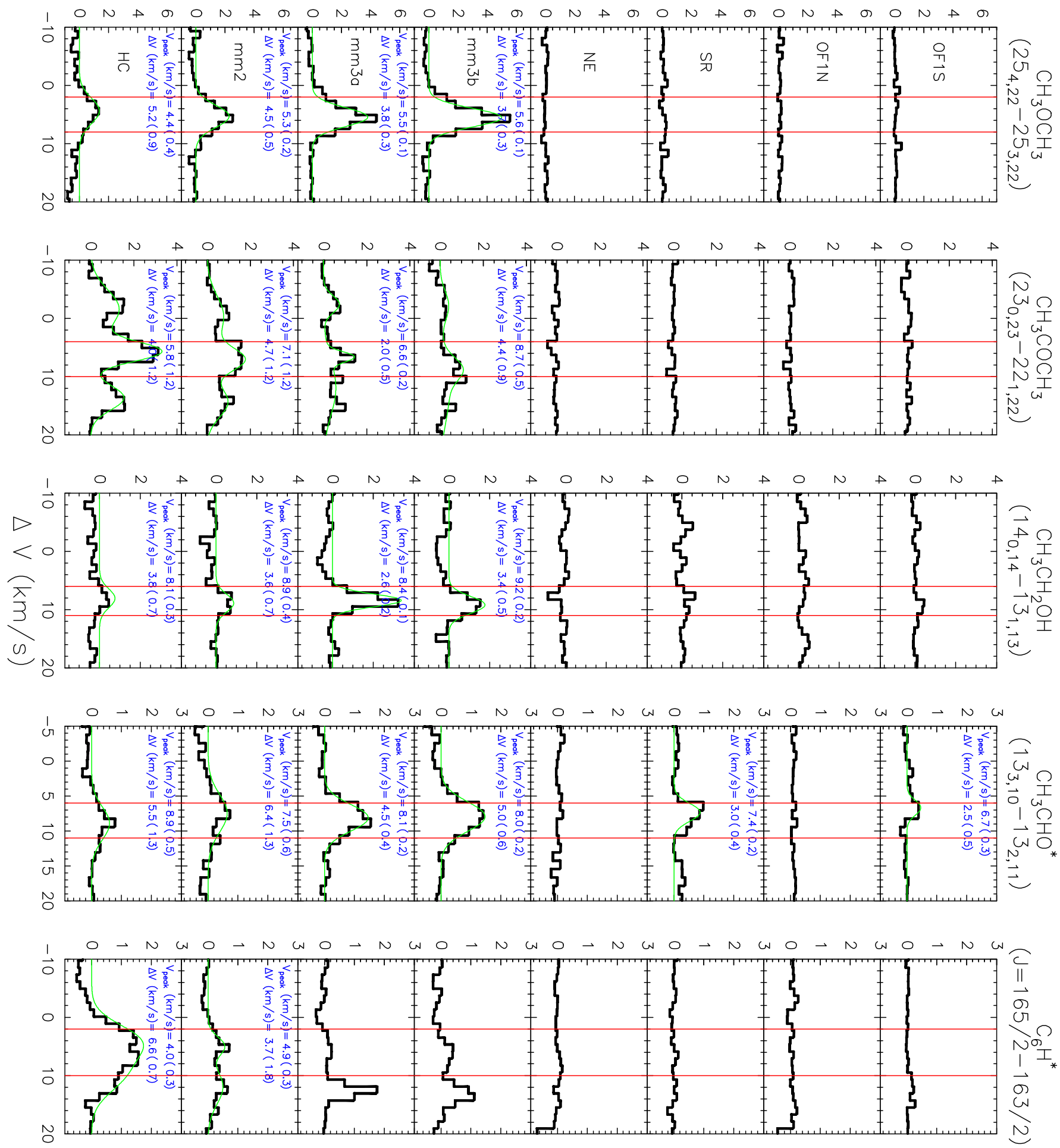

Fig. A.3. continued. 
A\&A 581, A71 (2015)

(Jy/beam)
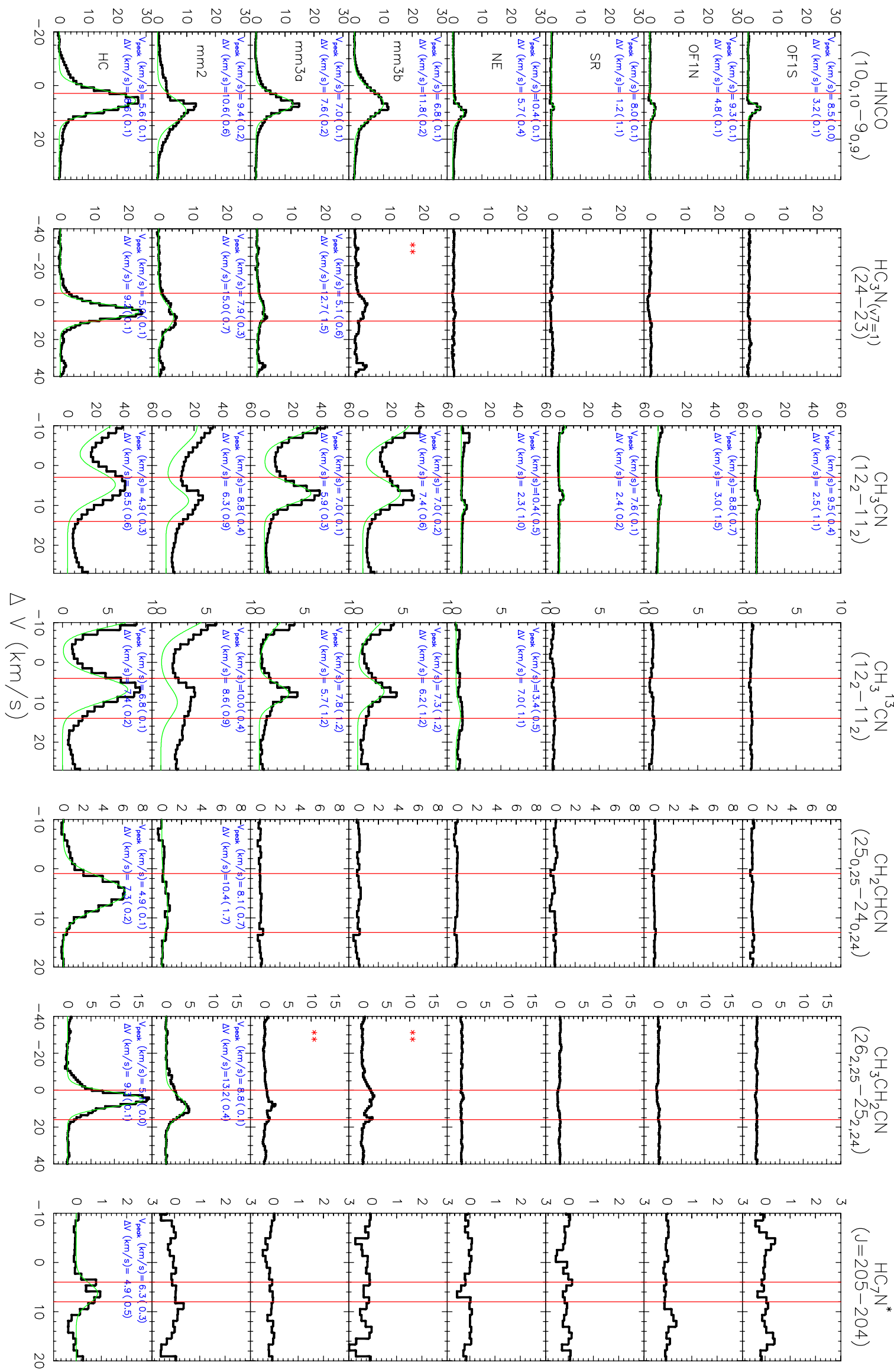

Fig. A.3. continued. 
S. Feng et al.: Resolving the chemical substructure of Orion-KL

(Jy/beam)
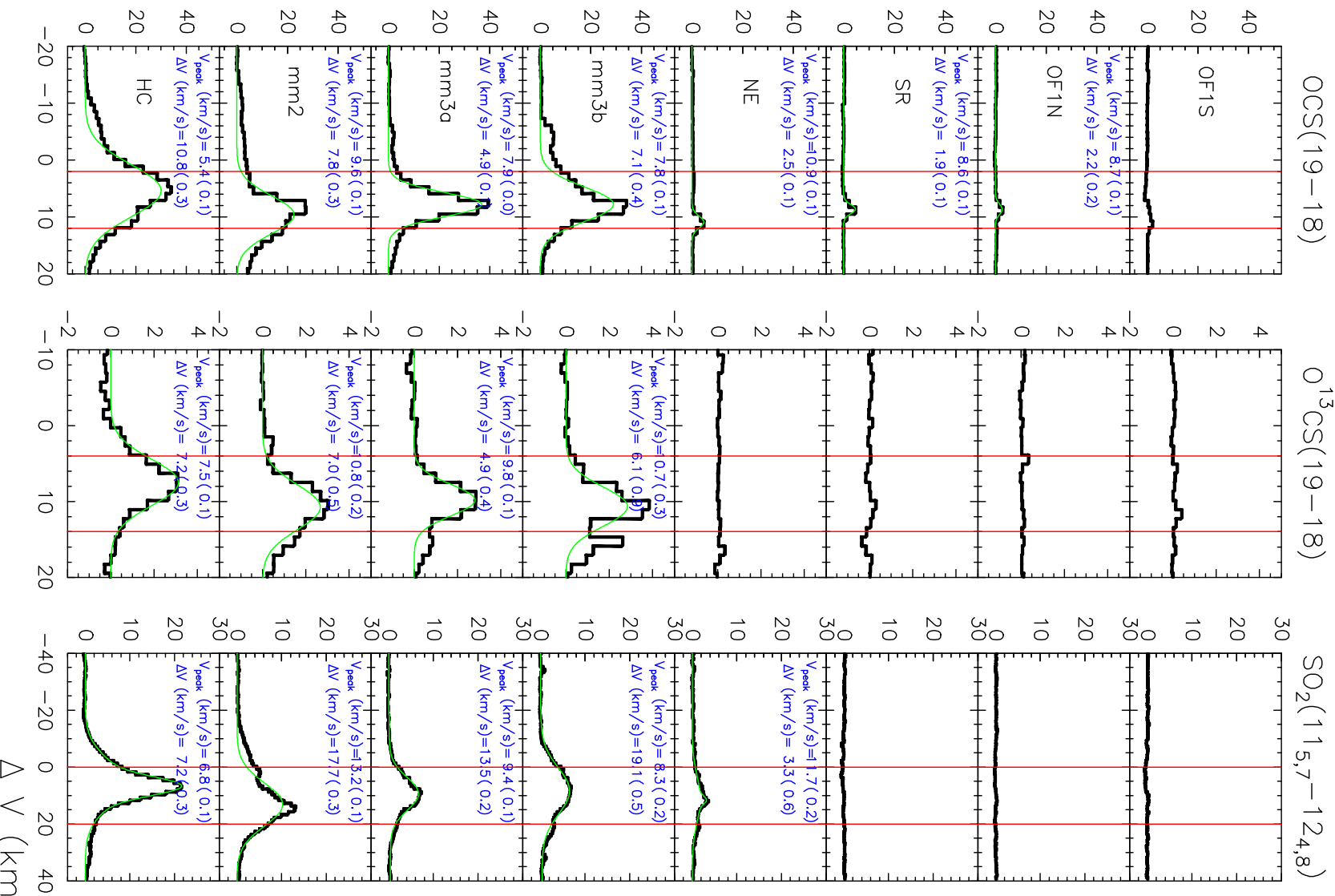

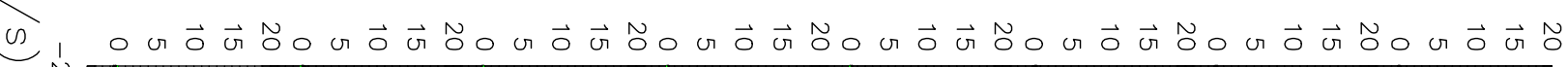
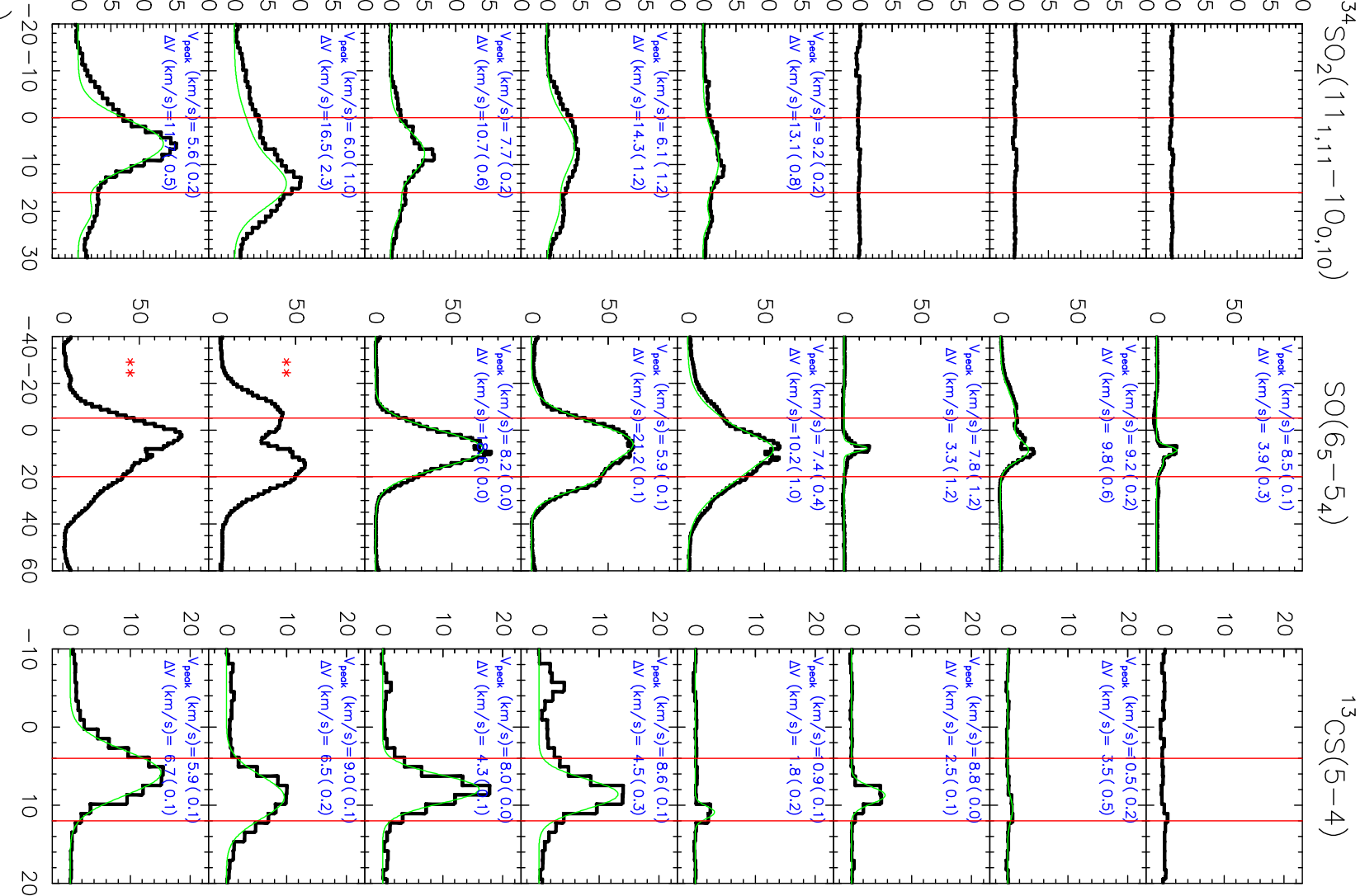

Fig. A.3. continued. 


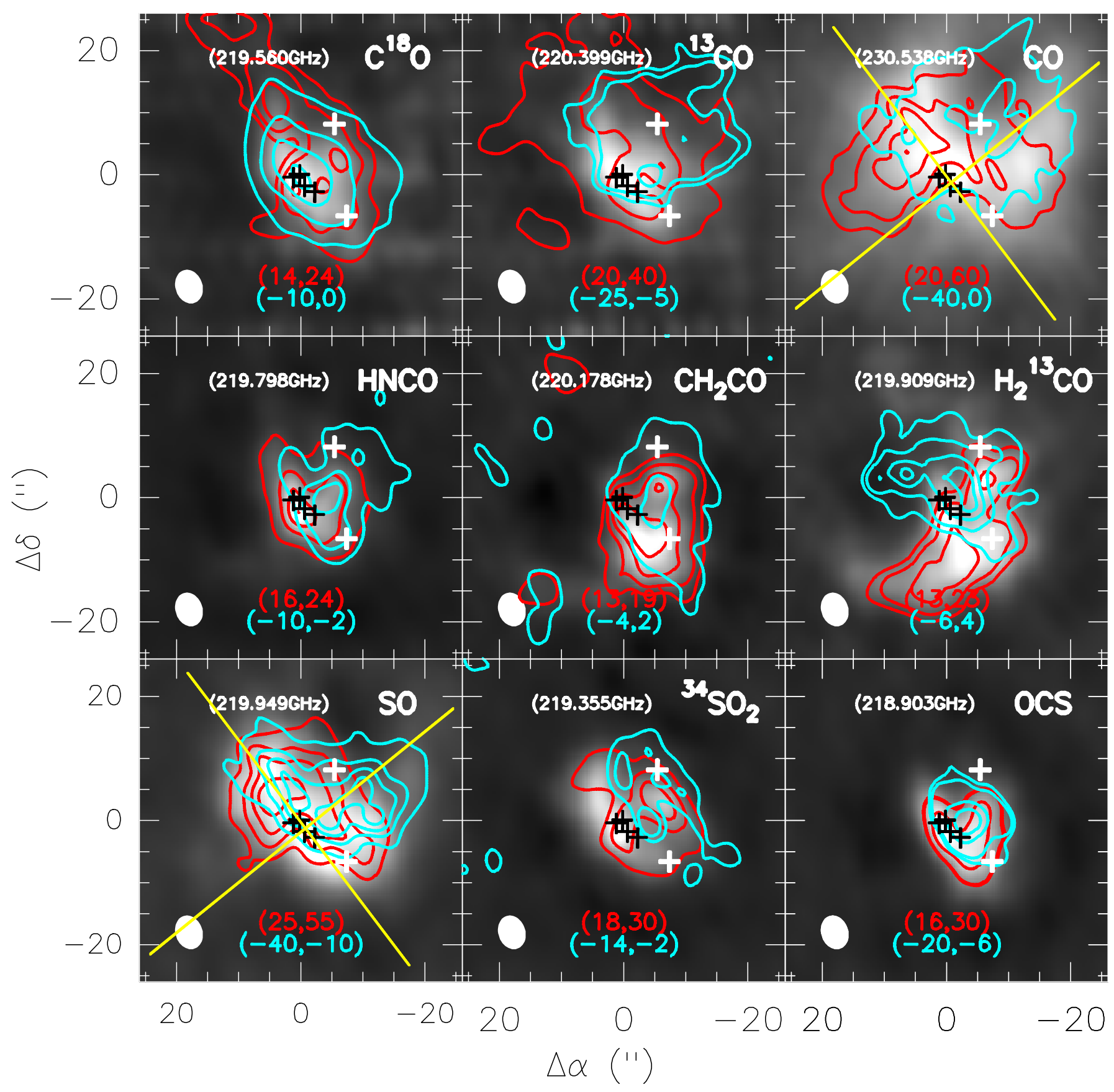

Fig. A.4. Line wing flux integration maps from the combined SMA-30 m data at $1.3 \mathrm{~mm}$. The grey maps show the intensity integrated ranging through the main emission of their linewidth in Fig. A.3. Red and blue contours show the red-shifted and blue-shifted gas, with the intensity integrated as labelled. Yellow lines sketch the outflow directions. Black crosses denote the hotcore, SMA1, Source I, and Source N; white crosses denote the BN object and CR. 
S. Feng et al.: Resolving the chemical substructure of Orion-KL
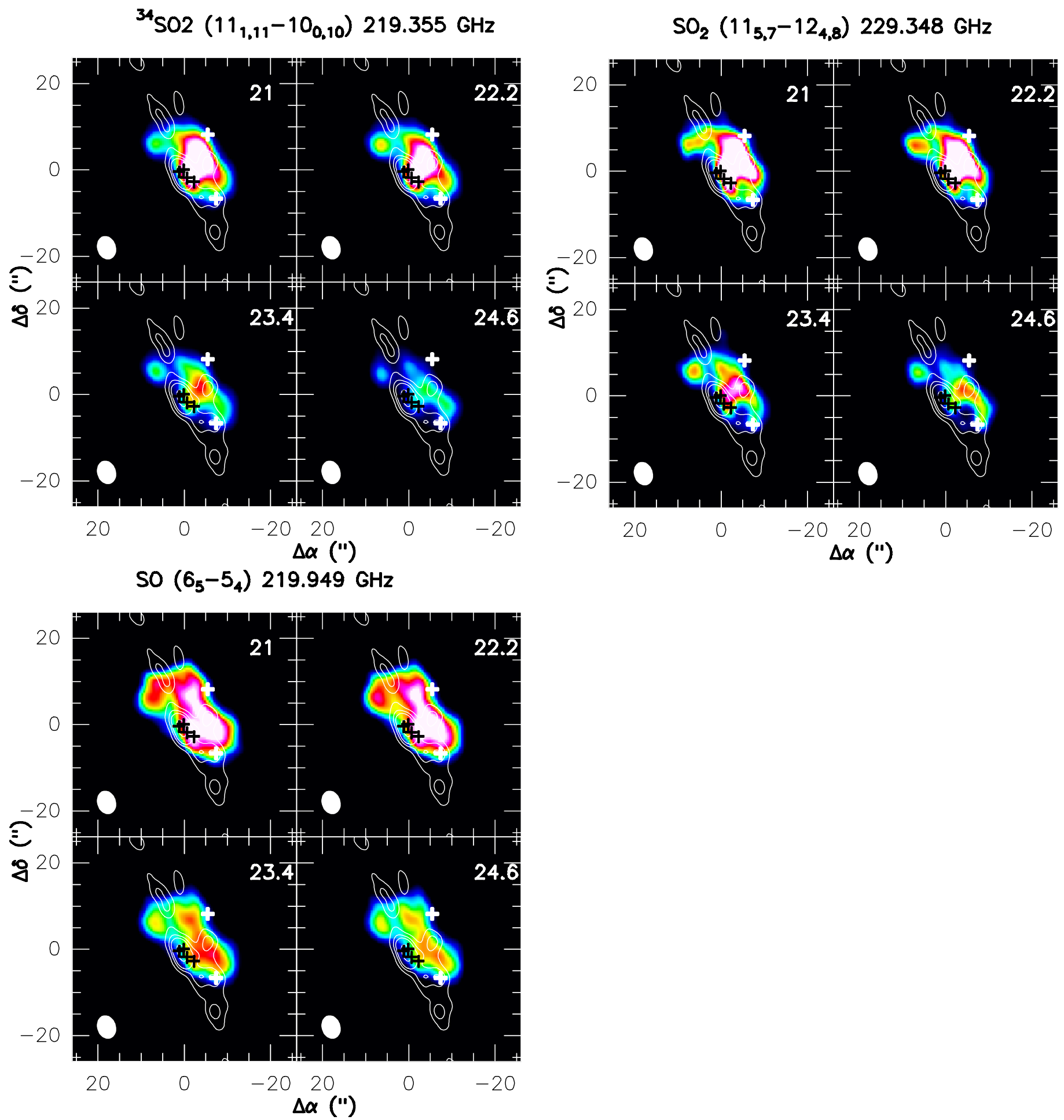

Fig. A.5. Channel maps of the second velocity component $\left(V_{\text {peak }} \sim 22 \mathrm{~km} \mathrm{~s}^{-1}\right)$ of the strongest unblended ${ }^{34} \mathrm{SO}_{2}$ and $\mathrm{SO}_{2}$ line, as well as of $\mathrm{SO}\left(6_{5} \rightarrow 5_{4}\right)$. 
$\mathrm{CH}_{3} \mathrm{OH}\left(8_{0,8}-7,6\right) \mathrm{E} 220.078 \mathrm{GHz}$
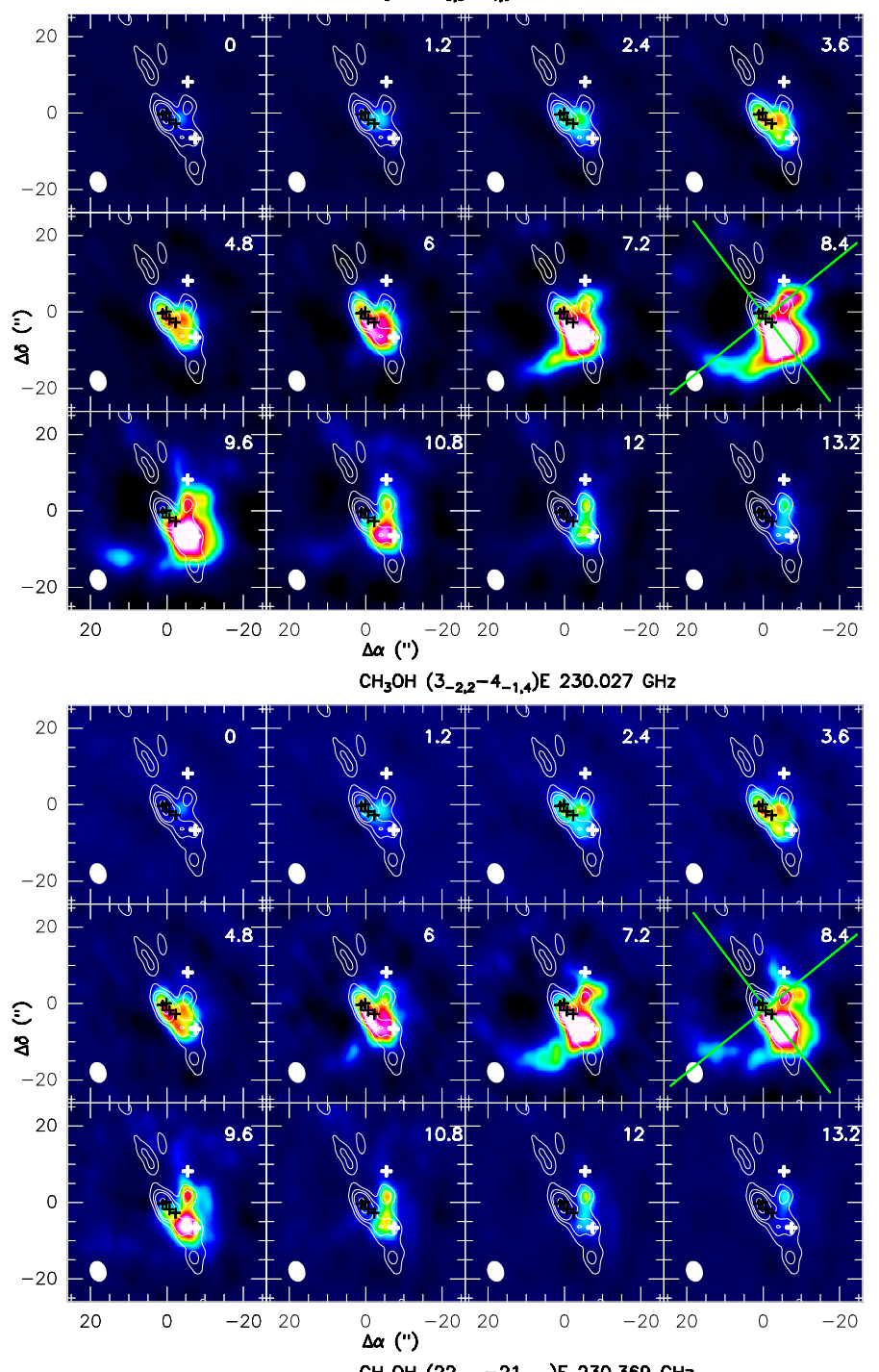

$\mathrm{CH}_{3} \mathrm{OH}\left(22_{4,18}-21_{5,17}\right) \mathrm{E} 230.369 \mathrm{GHz}$

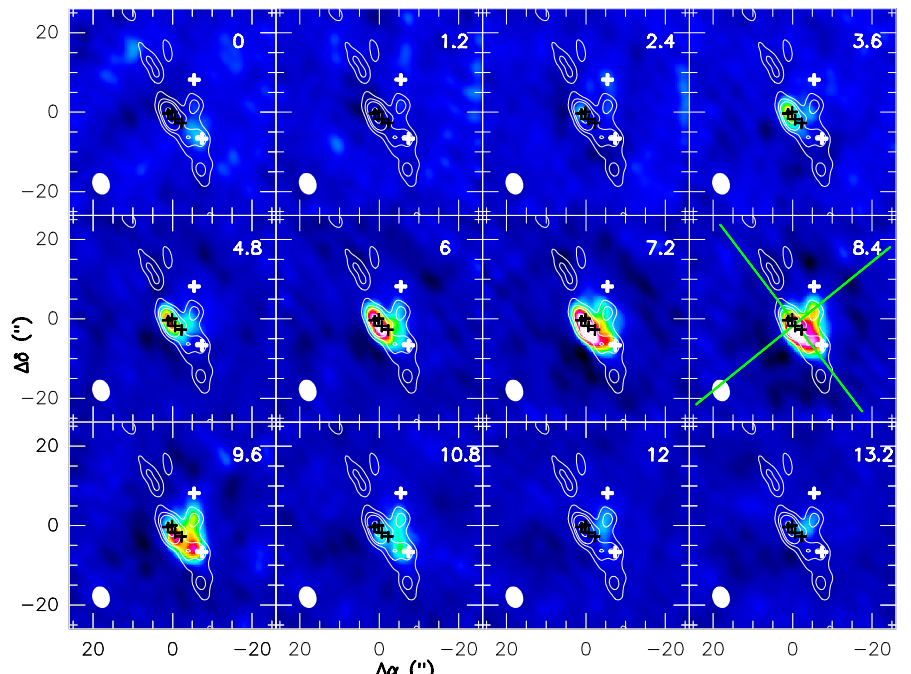

$\mathrm{CH}_{3} \mathrm{OH}\left(8_{-1,8}-7_{0,7}\right) \mathrm{E} 229.759 \mathrm{GHz}$

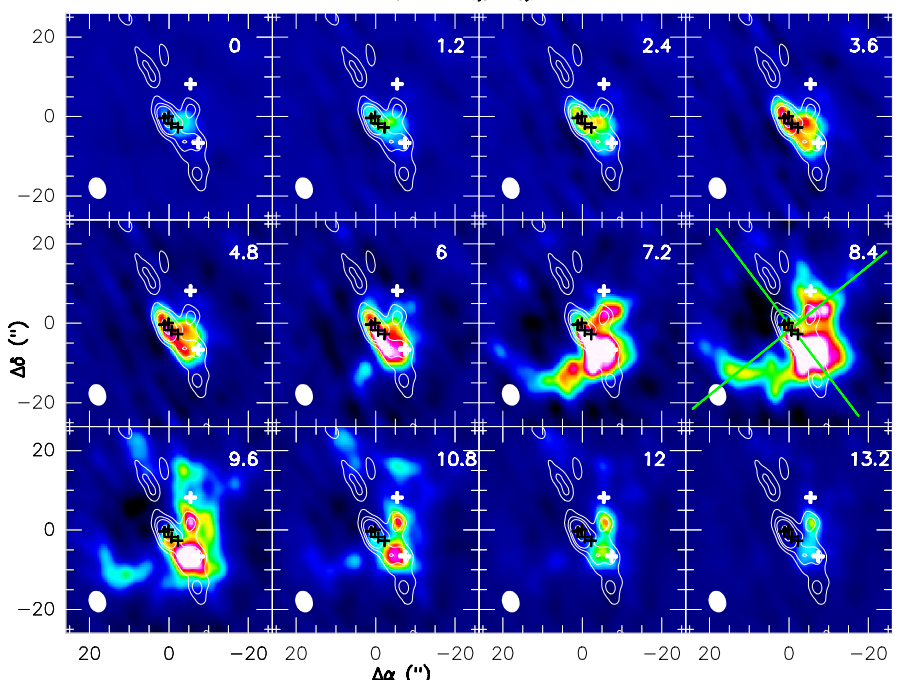

$\mathrm{CH}_{3} \mathrm{OH}\left(15_{4,11}-16_{3,13}\right) \mathrm{E} 229.589 \mathrm{GHz}$

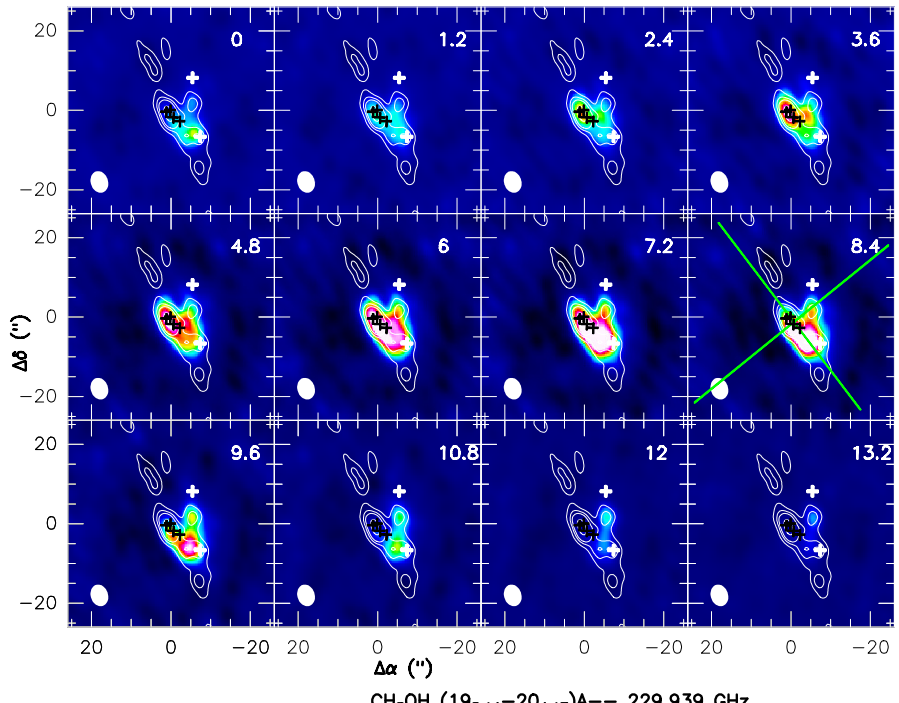

$\mathrm{CH}_{3} \mathrm{OH}\left(19_{5,14}-2 \mathrm{O}_{4,17}\right) \mathrm{A}--229.939 \mathrm{GHz}$

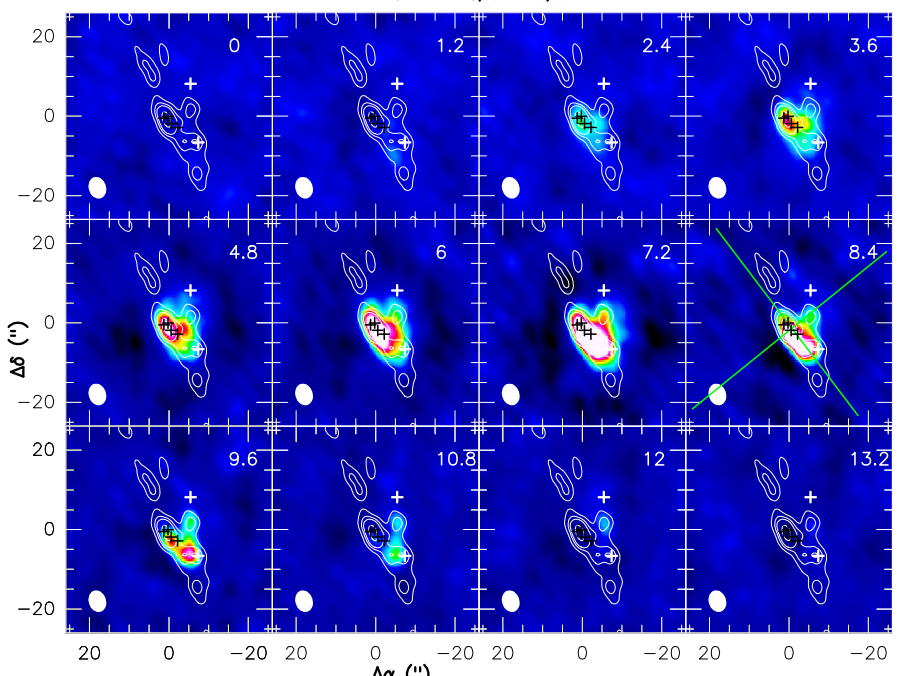

Fig. A.6. Channel maps of unblended $\mathrm{CH}_{3} \mathrm{OH}$ lines show multiple velocity-dependent emission peaks: $2.4-3.6 \mathrm{~km} \mathrm{~s}^{-1}$ towards SMA1, $\sim 4.8-6 \mathrm{~km} \mathrm{~s}^{-1}$ towards $\mathrm{HC}, 7.2-9.6 \mathrm{~km} \mathrm{~s}^{-1}$ towards $\mathrm{HC} / \mathrm{mm} 3 \mathrm{a} / \mathrm{mm} 3 \mathrm{~b}, 10.8 \mathrm{~km} \mathrm{~s}^{-1}$ towards $\mathrm{mm} 3 \mathrm{a} / \mathrm{mm} 3 \mathrm{~b} / \mathrm{mm} 2$ and $>12 \mathrm{~km} \mathrm{~s}{ }^{-1}$ towards mm2. Green lines sketch the outflow directions. Black crosses denote the hotcore, SMA1, Source I, and Source N; white crosses denote the BN object and the $\mathrm{CR}$. 